\title{
Observational signatures of particle acceleration in supernova remnants
}

\author{
E.A. Helder · J. Vink · A.M. Bykov . \\ Y. Ohira · J.C. Raymond · R. Terrier
}

the date of receipt and acceptance should be inserted later

\begin{abstract}
We evaluate the current status of supernova remnants as the sources of Galactic cosmic rays. We summarize observations of supernova remnants, covering the whole electromagnetic spectrum and describe what these observations tell us about the acceleration processes by high Mach number shock fronts. We discuss the shock modification by cosmic rays, the shape and maximum energy of the cosmic-ray spectrum and the total energy budget of
\end{abstract}

\section{E.A. Helder}

Department of Astronomy and Astrophysics, The Pennsylvania State University, 525 Davey Laboratory, University Park, PA 16802, USA

E-mail: helder@psu.edu

J. Vink

Astronomical Institute Anton Pannekoek, Universiteit van Amsterdam,

Postbus 94249, 1090 GE Amsterdam, the Netherlands

E-mail: j.vink@uva.nl

\section{A.M. Bykov}

Ioffe Institute for Physics and Technology, 194021 St. Petersburg, Russia

E-mail: byk@astro.ioffe.ru

St.Petersburg State Politechnical University

Y. Ohira

Theory Center, Institute of Particle and Nuclear Studies, KEK (High Energy Accelerator Research Organization), 1-1 Oho, Tsukuba 305-0801, Japan

E-mail: ohira@post.kek.jp

J.C. Raymond

Harvard-Smithsonian Center for Astrophysics, 60 Garden Street, Cambridge, MA 02138, USA

E-mail: jraymond@cfa.harvard.edu

R. Terrier

Astroparticule et Cosmologie, Universitè Paris7/CNRS/CEA, Batiment Condorcet, 75013 Paris, France

E-mail: rterrier@in2p3.fr 
cosmic rays in and surrounding supernova remnants. Additionally, we discuss problems with supernova remnants as main sources of Galactic cosmic rays, as well as alternative sources.

Keywords Supernova remnants $\cdot$ cosmic rays $\cdot$ acceleration of particles

\section{Introduction}

This year, we celebrate the discovery of cosmic rays (CRs) a century ago. In 1912, Victor Hess went up in the atmosphere with a balloon, and found that the strength of ionizing radiation increased as he went up (Hess, 1912). He realized that this increase points towards a cosmic origin of the radiation. For this reason the radiation was dubbed 'cosmic rays'. Later, it was found that these CRs were in fact energetic particles, rather than electromagnetic radiation. However, 'cosmic rays' is, although not an adequate description, still the currently used name for these particles.

Since their discovery, CRs have been subject to intensive study. For a good reason, as CRs can have tremendous energies: particles with energies more than $10^{20} \mathrm{eV}$ have been detected (Figure 1). Additionally, their energy density in the interstellar medium is about one third of the total energy density in the interstellar medium, comparable to the thermal and magnetic energy densities. Moreover, CRs provide an additional source of observational information on their accelerators next to electromagnetic radiation.

Supernova remnants have been the main candidates for accelerating Galactic CRs for decades (Baade and Zwicky, 1934), but this is still subject to debate. In this paper, we describe the current observational status of CRs in SNRs. We describe the emission directly originating from the CRs as well as how we can get information on CR acceleration in remnants from other observational characteristics. Also, we briefly discuss the problems with supernova remnants as main sources of Galactic CRs as well as alternative sources.

\subsection{Supernovae and their remnants as sources of Galactic cosmic rays}

Particles with energies less than $10^{18} \mathrm{eV}$ have gyro radii smaller than the thickness of the Galactic disk. Hence, particles with higher energies are likely to escape the Galaxy, indicating that the CRs well above $10^{18} \mathrm{eV}$ are probably accelerated outside of the Galaxy. In contrast, the particles with lower energies are contained within the Galactic magnetic field. The trajectories of these particles are curved and randomized by the magnetic field, causing their direction of arrival at Earth to be largely uncorrelated with the direction to their sources.

One clue towards the sources of Galactic CRs is the shape of the $\mathrm{CR}$ spectrum. The constant power-law slope of 2.7 over many decades in energy likely indicates that the particles of different energies were accelerated by a similar mechanism. As this is a steep power law, most of the energy in CRs is contributed by CRs with low $(\mathrm{GeV})$ energies. Unfortunately, the spectrum at 
these lower energies is heavily modified by solar modulation. Therefore, it is hard to determine the total energy budget in CRs from experiments directly. Nevertheless, Webber (1998) reports an CR energy density in the Galaxy of $1.8 \mathrm{eV}$ per cubic centimeter, correcting for solar modulation effects by using Voyager and Pioneer data. Isotope studies show that CRs with $\mathrm{GeV}$ energies diffuse out of the Galaxy in 15.0土 1.6 Myr (Yanasak et al., 2001). Galactic supernova explosions can replenish these losses, provided that they transfer $\sim 10-20 \%$ of their kinetic energy $\left(E_{\mathrm{SN}}=10^{51} \mathrm{erg}\right)$ into CRs (Hillas, 2005), assuming two to three supernova explosions per century (Tammann et al. 1994).

During a supernova explosion, the ejecta are expelled into the surrounding medium with speeds as large as tens of thousands of kilometers per second. The ejecta carry the kinetic energy of the supernova explosion and cause high Mach number shock fronts in the interstellar medium. At the end of the seventies, four independent papers proposed an acceleration mechanism for charged particles that operates at high Mach number shocks (Axford et al. 1977, Krymskii, 1977, Bell, 1978, Blandford and Ostriker, 1978). This mechanism concerns particles scattering up and down the shock front on turbulent magnetic fields, thereby effectively gain energy each time they cross the shock front. This process, called 'diffusive shock acceleration', naturally produces energetic particles, with a power-law spectrum with index 2 (see Malkov and

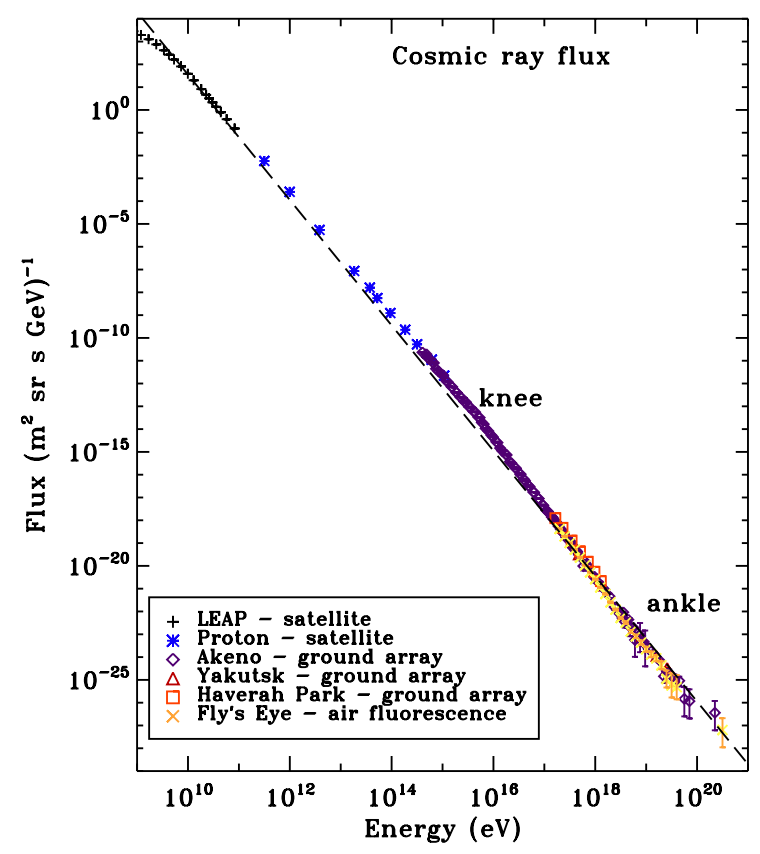

Fig. 1 Cosmic-ray spectrum as observed by different experiments. Data compiled by J. Swordy, courtesy to Klara Schure. 


\begin{tabular}{|c|c|c|c|}
\hline Name & $\begin{array}{l}\mathrm{GeV} \\
\gamma \text {-rays }\end{array}$ & $\begin{array}{l}\mathrm{TeV} \\
\gamma \text {-rays }\end{array}$ & $\begin{array}{l}\text { age } \\
\text { [yr] }\end{array}$ \\
\hline Cassiopeia A & $\mathrm{Y}^{1}$ & $\mathrm{Y}^{2}$ & $330^{3}$ \\
\hline $\mathrm{G} 1.9+0.3$ & $\mathrm{~N}$ & $\mathrm{~N}$ & $\sim 100^{4}$ \\
\hline HESS J1731-347 & $\mathrm{N}$ & $\mathrm{Y}^{5}$ & $\sim 1600^{5}$ \\
\hline Kepler & $\mathrm{N}$ & $\mathrm{N}$ & 408 \\
\hline RCW 86 & $\mathrm{~N}$ & $\mathrm{Y}^{6}$ & $1827^{7}$ \\
\hline RX J1713.7-3946 & $\mathrm{Y}^{8}$ & $\mathrm{Y}^{9}$ & $1626^{10}$ \\
\hline RX J0852.0-4622 & $\mathrm{Y}^{11}$ & $\mathrm{Y}^{12}$ & $1700-4300^{13}$ \\
\hline SN1006 & $\mathrm{N}$ & $\mathrm{Y}^{14}$ & 1006 \\
\hline SNR 0540-69.3 & $\mathrm{N}$ & $\mathrm{N}$ & $760-1660^{15}$ \\
\hline Tycho & $\mathrm{Y}^{16}$ & $\mathrm{Y}^{17}$ & 440 \\
\hline
\end{tabular}

Table 1 Table with shell-type supernova remnants that have X-ray synchrotron emission, together with their properties at other wavelenths. ${ }^{1}$ Abdo et al. (2010b), 2 Albert et al. (2007b); Acciari et al. (2010), $\sqrt[3]{\text { Fesen et al. }(2006),{ }^{4} \text { Reynolds }}$ et al. (2008), ${ }^{5}$ H.E.S.S. Collaboration et al. (2011), ${ }^{6}$ Aharonian et al. (2009),

${ }^{7}$ Stephenson and Green (2002), ${ }^{\circ}$ Abdo and Fermi LAT Collaboration (2011), ${ }^{9}$ Aharonian et al. (2004), ${ }^{10}$ Wang et al. (1997), ${ }^{11}$ Tanaka et al. (2011), ${ }^{12}$ HESS Collaboration: F. A. Aharonian (2006), ${ }^{13}$ Katsuda et al. $(2008),{ }^{14}$ Acero et al. (2010), ${ }^{15}$ Park et al. (2010), ${ }^{16}$ Giordano et al. (2011), ${ }^{17}$ Acciari et al. (2011)

Drury, 2001; Schure et al., 2012, for a review). The somewhat softer CR spectrum observed in the Earth's, might be explained by propagation effects of CRs through the Galaxy.

As the direction of arrival of Galactic CR is essentially uncorrelated to their sources, most of the observations on the sources of Galactic CRs, comes from electromagnetic radiation. As energetic electrons gyrate in the local magnetic field, they emit synchrotron emission. This synchrotron emission is observed at radio wavelengths in supernova remnants, revealing the presence of GeV electrons (Shklovskii, 1953, Minkowski, 1957). Also, X-ray synchrotron emitting $\mathrm{TeV}$ electrons were identified at the shock fronts of the SN 1006 supernova remnant and others (Koyama et al., 1995, and see section 2.3 . In the last decade, $\mathrm{GeV}$ and $\mathrm{TeV} \gamma$-ray emission has been observed from several supernova remnants (Table 1.1), indicating the presence of particles with $\mathrm{GeV}$ and $\mathrm{TeV}$ energies (e.g., Aharonian et al., 2004, Abdo et al., 2010b and see section 2.13.

\subsection{Different types of supernova explosions}

Classification of supernova explosions is traditionally based on the presence of hydrogen lines in the optical spectrum at maximum light. A supernova with hydrogen lines in its spectrum is classified as a type II, and without is classified as a type I (Minkowski, 1941). More specifically, a spectrum without hydrogen lines but with strong Si II lines is classified as a type Ia spectrum, without Si II lines but with strong helium I lines as type Ib and if the spectra are lacking both strong Si II and He I lines, the supernova is classified as a type Ic. Type II supernovae have subclassifications as well, depending on their light curves. A supernova explosion with a plateau in its light curve is a 
type IIP, one with a linear phase is a type IIL (Barbon et al. 1979). Another subcategory is type IIb supernovae: their spectrum shows a weak hydrogen line shortly after the explosion, which is absent in the second maximum of the lightcurve (Filippenko, 1988). Recently, spectra of light echoes of the explosion that resulted in the Cassiopeia A remnant (Cas A) showed that this was a type IIb supernova (Krause et al., 2008). A fourth category of supernova explosions are type IIn supernovae (Schlegel, 1990). Type IIn supernovae are characterized by narrow emission lines in their spectra, probably produced by the interaction of the ejecta with dense circumstellar medium around these explosions, expelled by the progenitor star before explosion. For a more extensive description of observational characteristics of supernova explosions, see Filippenko (1997). About $24 \%$ of all supernovae are type Ia's, $10 \%$ are type Ibc's and 57\% are type II's (Li et al., 2011).

Apart from this observational classification scheme, we now know that type Ia supernovae are related to thermonuclear explosions of white dwarfs (first suggested by Hoyle and Fowler, 1960). White dwarfs explode when they reach the Chandrasekhar mass of $1.38 M_{\odot}$ by accreting material from a binary companion. Although the link from type Ia supernovae to thermonuclear explosions of white dwarfs is fully canonized, it is rather unclear which progenitor systems (single or double degenerate binaries) create these explosions. More specifically, it is unclear which systems accrete efficient enough to explain the observed frequency of type Ia explosions (see Hillebrandt and Niemeyer, 2000, for a review).

The other (non type Ia) explosions mark the deaths of massive $(M \gtrsim$ $\left.8 M_{\odot}\right)$ stars. These are called core collapse supernovae. After these stars have burned all the nuclear energy in their cores, they are left with an iron core. The gravitational pressure will result in an implosion of this iron core to a neutron star or a black hole. The envelope will bounce onto the core into the circumstellar medium with tens of thousands kilometers per second.

Although the explosion mechanisms for core collapse and thermonuclear explosions are quite different, the kinetic energies dumped into the ambient medium are reasonably similar: $\sim 10^{51} \mathrm{erg}$.

\subsection{Supernova remnant evolution}

After a star goes supernova, the stellar ejecta expand into the ambient medium. These expanding ejecta will create a shock front, the forward shock, which will slow down, as it sweeps up an increasing amount of ambient medium. As a reaction, a second shock will develop, moving away from the forward shock, into the stellar ejecta. This second shock is called the reverse shock. The evolution of supernova remnants is characterized by the free expansion phase, the Sedov phase and the radiative phase (in chronological order). Initially, the forward shock velocity is approximately constant as long as the swept-up mass is smaller than the ejecta mass (free expansion phase). Precisely speaking, the forward shock velocity slightly decelerates during the free expansion phase because it transfers kinetic energy into sweeping up the ambient medium (Chevalier, 1982b). In the subsequent phase (Sedov phase) most energy has transferred from free expanding ejecta to the shock-heated 
shell. As radiative cooling is still negligible in this phase, the supernova remnant expands adiabatically in this phase. As the shock slows down to velocities below $200 \mathrm{~km} \mathrm{~s}^{-1}$, the post-shock temperature will be less than $5 \times 10^{5}$ $\mathrm{K}$. This will cause the radiation of $\mathrm{H}, \mathrm{He}, \mathrm{C}, \mathrm{N}$ and $\mathrm{O}$ line emission to increase drastically (Raymond, 1979; Draine and McKee, 1993, Schure et al., 2009), and therewith radiative cooling becomes significant (radiative phase). The length of each evolution phase of supernova remnants depends on supernova type and structure of their surroundings.

For type Ia supernovae, the surrounding medium typically has a constant density ISM with $n_{0} \sim 1 \mathrm{~cm}^{-3}$ and $T_{0} \sim 1 \mathrm{eV}$, where $n_{0}$ and $T_{0}$ are the number density and the temperature of ISM, respectively. A mass $M_{\mathrm{ej}} \sim$ $1 M_{\odot}$ ejected with $E_{\mathrm{SN}} \sim 10^{51}$ erg in a supernova explosion has an initial velocity $\left(V_{0}\right)$ of

$V_{0}=\sqrt{\frac{2 E}{M_{\mathrm{ej}}}}=10^{9} \mathrm{~cm} \mathrm{~s}^{-1}\left(\frac{E_{\mathrm{SN}}}{10^{51} \mathrm{erg}}\right)^{1 / 2}\left(\frac{M_{\mathrm{ej}}}{1 M_{\odot}}\right)^{-1 / 2}$.

The forward-shock velocity during the free expansion phase is $V_{\mathrm{s}} \sim V_{0}$, so that the Mach number is given by

$\mathcal{M}=\frac{V_{\mathrm{s}}}{c_{\mathrm{s}}} \sim 10^{3}\left(\frac{E_{\mathrm{SN}}}{10^{51 \mathrm{erg}}}\right)^{1 / 2}\left(\frac{M_{\mathrm{ej}}}{1 M_{\odot}}\right)^{-1 / 2}\left(\frac{T_{0}}{1 \mathrm{eV}}\right)^{-1 / 2}$,

where $c_{\mathrm{s}}=10^{6} \mathrm{~cm} \mathrm{~s}^{-1}\left(T_{0} / 1 \mathrm{eV}\right)$ is the sound speed. The ejecta start to decelerate when the swept-up mass $4 \pi \rho_{0} R_{\mathrm{s}}^{3} / 3$ becomes comparable to the ejecta mass, where $R_{\mathrm{s}}$ and $\rho_{0}$ are the forward shock radius and the density of the ambient medium, respectively. The transition from the free expansion phase to the Sedov phase occurs at shock radius $R_{\text {Sedov }}$,

$R_{\text {Sedov }}=\left(\frac{3 M_{\mathrm{ej}}}{4 \pi \rho_{0}}\right)^{1 / 3}=2.1 \mathrm{pc}\left(\frac{M_{\mathrm{ej}}}{1 M_{\odot}}\right)^{1 / 3}\left(\frac{n_{0}}{1 \mathrm{~cm}^{-3}}\right)^{-1 / 3}$,

and at time $t_{\text {Sedov }}$,

$t_{\text {Sedov }}=\frac{R_{\text {Sedov }}}{V_{0}}=210 \mathrm{yr}\left(\frac{E_{\mathrm{SN}}}{10^{51} \mathrm{erg}}\right)^{-1 / 2}\left(\frac{M_{\mathrm{ej}}}{1 M_{\odot}}\right)^{5 / 6}\left(\frac{n_{0}}{1 \mathrm{~cm}^{-3}}\right)^{-1 / 3}$.

For supernova remnants in superbubbles, $R_{\text {Sedov }}$ and $t_{\text {Sedov }}$ are about ten times larger than that in a typical ISM because the density is $n_{0} \sim 10^{-3} \mathrm{~cm}^{-3}$.

After $t_{\text {Sedov }}$, the supernova remnant evolution is in the Sedov phase as long as the radiative cooling is negligible. Assuming that the swept-up mass is larger than the ejecta mass and the total explosion energy is conserved,

$\frac{1}{2} V_{\mathrm{s}}^{2} \times \frac{4 \pi}{3} \rho_{0} R_{\mathrm{s}}^{3}=$ const. ,

the evolution of the forward shock, $R_{\mathrm{s}}$ and $V_{\mathrm{s}}$ are given by

$R_{\mathrm{s}}=R_{\text {Sedov }}\left(\frac{t}{t_{\text {Sedov }}}\right)^{2 / 5}$, 
$V_{\mathrm{s}}=\frac{\mathrm{d} R_{\mathrm{s}}}{\mathrm{d} t}=\frac{2 R_{\text {Sedov }}}{5 t_{\text {Sedov }}}\left(\frac{t}{t_{\text {Sedov }}}\right)^{-3 / 5}$.

A simple treatment of the dynamics of the forward shock from the free expansion to the Sedov phase has been given by Ostriker and McKee (1988), Bisnovatyi-Kogan and Silich (1995) and Truelove and McKee (1999).

When the supernova remnant age becomes comparable to the radiative cooling time, the supernova remnant evolution changes from the Sedov phase to the radiative phase (the pressure driven snowplow phase and the momentum-conserving snowplow phase). This transition occurs at $t_{\mathrm{tr}}$, is given by (Petruk, 2005)

$t_{\mathrm{tr}}=2.8 \times 10^{4} \mathrm{yr}\left(\frac{E_{\mathrm{SN}}}{10^{51} \mathrm{erg}}\right)^{4 / 17}\left(\frac{n_{0}}{1 \mathrm{~cm}^{-3}}\right)^{-9 / 17}$.

The asymptotic supernova remnant evolution of the radiative phase was investigated by McKee and Ostriker (1977) $\left(R_{\mathrm{s}} \propto t^{2 / 7}\right.$ for the pressure driven snowplow phase) and Oort (1951) $\left(\overline{R_{\mathrm{s}}} \propto t^{1 / 4}\right.$ for the momentum-conserving snowplow phase). Bandiera and Petruk (2004) found more accurate analytical solutions for the radiative phase. Truelove and McKee (1999) also provided approximate solutions for the reverse shock evolution. For uniform ejecta and a uniform ISM, the reverse shock position, $R_{\mathrm{r}}$, and the velocity of the reverse shock in the rest frame of the unshocked ejecta, $V_{1}$ ] is given by (Truelove and McKee, 1999)

$$
\begin{aligned}
R_{\mathrm{r}}= & R_{\text {Sedov }}\left(\frac{t}{t_{\text {Sedov }}}\right) \times \\
& \left\{\begin{array}{ll}
1.29\left\{1+0.947\left(\frac{t}{t_{\text {Sedov }}}\right)^{-2 / 3}\right\} & \left(t<t_{\text {Sedov }}\right) \\
0.707\left(1.22-0.0465\left(\frac{t}{t_{\text {Sedov }}}\right)-0.533 \ln \left(\frac{t}{t_{\text {Sedov }}}\right)\right) & \left(t>t_{\text {Sedov }}\right)
\end{array},\right.
\end{aligned}
$$

$$
\begin{aligned}
& V_{\mathrm{r}}=\frac{R_{\text {Sedov }}}{t_{\text {Sedov }}} \times \\
& \left\{\begin{array}{ll}
1.22\left(\frac{t}{t_{\text {Sedov }}}\right)^{3 / 2}\left\{1+0.947\left(\frac{t}{t_{\text {Sedov }}}\right)^{-5 / 3}\right\} & \left(t<t_{\text {Sedov }}\right) \\
0.377+0.0329\left(\frac{t}{t_{\text {Sedov }}}\right) & \left(t>t_{\text {Sedov }}\right)
\end{array} .\right.
\end{aligned}
$$

The velocity of the reverse shock in the rest frame of the unshocked ejecta, $V_{\mathrm{r}}$, increases with time for uniform ejecta. Truelove and McKee (1999) provided approximate solutions for some cases of type Ia supernovae evolution (see table 7 of Truelove and McKee, 1999).

\footnotetext{
1 Note that this is the velocity relevant for the physical conditions behind the shock front (outside of the reverse shock).
} 


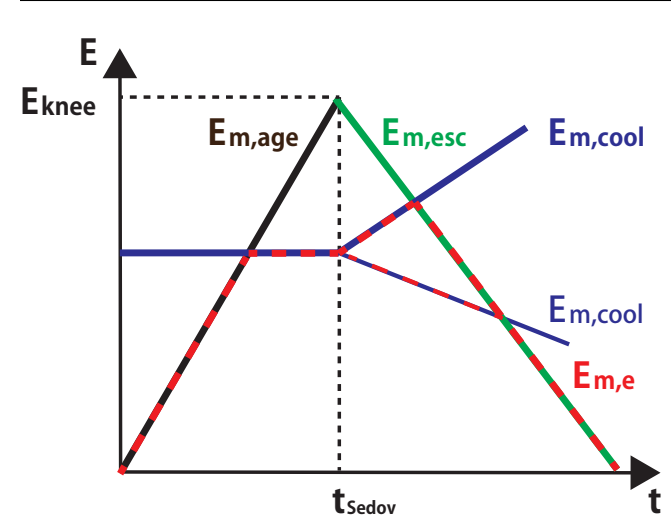

Fig. 2 Evolution of the maximum energy. The black, blue and green lines show the age-limited, the cooling-limited and the escape-limited maximum energy, respectively. The red dashed lines show the evolution of the maximum energy of CR electrons. Thick and thin lines show cases where the cooling-limited maximum energy increases or decreases with time during the Sedov phase, respectively.

For core-collapse supernovae, the structure of surrounding medium is more complicated and depends on the details of the stellar mass loss history, which in itself depends on the initial mass, binarity and local ambient medium density (e.g. van Marle et al., 2004). Roughly speaking, in order of increasing distance to the center, the ambient material is organized by the dense red supergiant wind, a shocked low density wind created during the main sequence stage, and interstellar medium. The density structure in the dense red supergiant wind is $\rho_{0} \propto r^{-2}$ whereas density structures of other regions are constant. Approximate solutions of supernova remnant evolution in these media are also obtained in the same manner as type Ia supernovae (e.g. Chevalier, 1982a: Truelove and McKee, 1999; Ostriker and McKee, 1988; Bisnovatyi-Kogan and Silich, 1995, Laming and Hwang, 2003).

\subsection{Cosmic-ray acceleration efficiency during supernova remnant evolution}

As mentioned above, there are three phases for supernova remnant evolution. Although we do not understand when particle acceleration stops, observations tell us that relativistic electrons are accelerated during the free expansion phase and the Sedov phase. Particle acceleration mechanisms during the radiative phase have not been investigated in detail. However, these are not expected to provide a significant contribution to the Galactic CR density, as the shock velocity is low, and a substantial fraction of the shock energy is lost by radiative losses.

The maximum energy of $\mathrm{CR}$ protons is limited by the finite age or the escape condition. According to the theory of diffusive shock acceleration, an estimate for the typical time to accelerate a particle with an elementary charge to an energy $E$ is (Lagage and Cesarsky, 1983; Jokipii, 1987, Parizot 
et al. 2006):

$t_{\mathrm{acc}} \approx 1.8 \frac{D_{2}}{V_{\mathrm{s}}^{2}} \frac{3 r^{2}}{r-1}=124 \eta_{\mathrm{g}} B_{-4}^{-1}\left(\frac{V_{\mathrm{s}}}{5000 \mathrm{~km} \mathrm{~s}^{-1}}\right)^{-2}\left(\frac{E}{100 \mathrm{TeV}}\right) \frac{r_{4}^{2}}{r_{4}-\frac{1}{4}} \mathrm{yr}$,

with $D_{2}$ the downstream diffusion coefficient for particles with energy $E$ (Drury, 1983), $V_{\mathrm{s}}$ the shock velocity, $B_{-4}$ the downstream magnetic field in units of $100 \mu \mathrm{G}, \eta_{\mathrm{g}}$ is the gyro factor and $r_{4}$ the overall compression ratio in units of 4 . The factor 1.8 comes from taking into account the difference between magnetic field upstream and downstream of the shock. Here, we assume that there is a highly turbulent, isotropic, magnetic field upstream and that the downstream magnetic field strength is determined by compression of the perpendicular component of the upstream magnetic field. Note that a higher compression ratio corresponds to a longer acceleration time, but that a high compression ratio itself is the result of efficient acceleration.

To get some understanding on the typical properties of particle acceleration we can approximate equation 11 by:

$t_{\mathrm{acc}}=\eta_{\mathrm{acc}} \frac{D}{V_{\mathrm{s}}^{2}}=\eta_{\mathrm{acc}} \eta_{\mathrm{g}} \frac{c E}{3 e B V_{\mathrm{s}}^{2}}$

where $\eta_{\text {acc }}$ is a numerical factor which depends on the shock compression ratio and $D$ is the diffusion coefficient around the shock (Drury, 1983). The diffusion coefficient relates to the mean free path $(\lambda)$ as follows: $D=\frac{1}{3} \lambda c$, where $c$ is the speed of light. We generally express $\lambda$ in terms of gyroradius $\left(r_{\mathrm{g}}\right): \lambda=r_{\mathrm{g}} \eta_{\mathrm{g}}$. The lower $\eta_{\mathrm{g}}$, the more turbulent the magnetic field. $\eta_{\mathrm{g}}=1$ is generally referred to as Bohm diffusion.

One can obtain the age-limited maximum energy, $E_{\mathrm{m}, \text { age }}$, from the condition $t_{\mathrm{acc}}=t$ assuming $r_{4}=1$

$E_{\mathrm{m}, \text { age }}=\frac{3 e B V_{\mathrm{s}}^{2} t}{\eta_{\mathrm{acc}} \eta_{\mathrm{g}} c}$

The escape time due to the diffusion, $t_{\mathrm{esc}}$, is written by

$t_{\mathrm{esc}}=\eta_{\mathrm{esc}} \frac{R_{\mathrm{s}}^{2}}{D}=\frac{\eta_{\mathrm{esc}}}{\eta_{\mathrm{g}}} \frac{3 e B R_{\mathrm{s}}^{2}}{c E}$,

where $\eta_{\mathrm{esc}}$ is a numerical factor $\left(\eta_{\mathrm{esc}}<1\right)$. Note that $t_{\mathrm{esc}}$ is similar to $t_{\mathrm{age}}$ for $\sqrt{\eta_{\mathrm{esc}} \eta_{\mathrm{acc}}} \approx 1$ and $R_{\mathrm{s}} / V_{\mathrm{s}} t \approx 1$.

For this reason, the escape-limited maximum energy is similar to the age-limited maximum energy (Drury, 2011). The time dependence of the maximum energy $E_{\mathrm{m} \text {,age }} \approx E_{\mathrm{m} \text {,esc }}$ is given by

$E_{\mathrm{m}, \mathrm{esc}} \approx E_{\mathrm{m}, \text { age }} \propto \frac{B(t) V_{\mathrm{s}}(t)^{2} t}{\eta_{\mathrm{g}}(t)}$

where we assume that $\eta_{\text {acc }}$ is constant. For a constant density profile, the amplified magnetic field and their fluctuations are thought to be constant during 
the free expansion phase because the shock velocity is approximately constant. Therefore, the maximum energy linearly increases with time, $E_{\mathrm{m} \text {,age }} \propto$ $t$. After the free expansion phase $\left(t>t_{\text {Sedov }}\right)$, the shock velocity decreases with time, so that the maximum energy decreases with time $\left(E_{\mathrm{m}, \mathrm{esc}} \propto\right.$ $\left.B V_{\mathrm{s}}^{2} t \propto B t^{-1 / 5}\right)$. Additionally, the magnetic field is thought to decrease with time $\left(B \propto V_{\mathrm{s}}^{2}\right.$ or $\propto V_{\mathrm{s}}^{3}$, see section 2.7), leading to an even faster decrease of the maximum energy.

The number of CRs accelerated by supernova remnants increases with time since the swept-up mass increases with time. However, the detailed evolution of the number of CRs is unclear. Recently, several authors discuss the spectrum of escaping CRs corresponding to the source of Galactic CRs (Ptuskin and Zirakashvili, 2005, Ohira et al., 2010, Caprioli et al., 2010, Drury, 2011). They showed that the spectrum of escaping CRs depends on the evolution of the number of CRs and the evolution of the maximum energy, moreover, (Ptuskin and Zirakashvili, 2005) showed that the maximum energy to which particles can be accelerated differ for different types of supernovae.

\section{Observational diagnostics}

\subsection{Shock parameters}

As mentioned before, in order to maintain a steady Galactic CR energy density, on average 10-20\% of the explosion energy of a supernova has to be converted to CR energy. For these efficiencies, the acceleration process changes the shock structure and makes a precursor in the upstream region ${ }^{2}$ (Drury and Voelk, 1981). In the precursor region, magnetic-field amplification (Lucek and Bell, 2000, Bell, 2004, Ohira and Takahara, 2010, Bykov et al., 2011b, Schure and Bell, 2011; Reville and Bell, 2011) and plasma heating (McKenzie and Völk, 1982, Ghavamian et al., 2007; Rakowski et al., 2008, Niemiec et al., 2008; Ohira et al., 2009a; Raymond et al., 2011) are thought to occur. Although there is more and more evidence that magnetic field amplification is important, the process itself is not yet fully understood.

In the shock rest frame, the upstream plasma is decelerated by the CR pressure and and heated by plasma waves at the precursor region. As a result, the effective Mach number becomes small at the jump of the shock (subshock) and the compression ratio, at the subshock becomes less than four. On the other hand, the total compression ratio (the density ratio of the far upstream to the downstream) becomes large $(r>4)$. This is because a part of the downstream pressure is due a) to the relativistic particle with the adiabatic index of $\gamma=4 / 3(r=\gamma+1 / \gamma-1)$ and $b)$ escaping CRs take away shock energy.

The energy spectrum of accelerated particles also changes from that predicted by standard diffusive shock acceleration. The energy spectrum of low energy CRs becomes steeper than that of the standard diffusive shock acceleration because low energy CRs sample only the contrast in velocity across

\footnotetext{
2 The upstream region is the region in front of the shock, i.e. outside the remnant for the forward shock.
} 
the subshock $(r<4)$. On the other hand, the spectrum of high energy CRs becomes harder than that of the standard diffusive shock acceleration because high energy CRs sample the total velocity difference in total shock region (subshock plus shock precursor: $r>4$ ) because of the longer diffusion length (Berezhko and Ellison, 1999). As a result, the energy spectrum in wide energy range is not a single power law form (e.g. Berezhko and Ellison, 1999, Malkov and Drury, 2001, and section 2.11.

If a large fraction of the shock energy is converted to the CR energy, the mean temperature of the downstream plasma becomes smaller than that of the standard shock, $\langle T\rangle=3 \mu m_{\mathrm{p}} V_{\mathrm{s}}^{2} / 16$, where $\mu$ is the mean particle mass (see section 3.3.).

\subsection{Radio observations}

So far, about 300 supernova remnants have been observed in the radio band Green, 2009). Their spectra are nonthermal and their radio spectral indices ${ }^{3}$, $\alpha\left(S_{\nu} \propto \nu^{-\alpha}\right)$, are typically $\alpha=0.5 \pm 0.1$ (see figure 1 of Reynolds et al. 2011). Radio spectra can be interpreted as synchrotron radiation from relativistic electrons accelerated by diffusive shock acceleration. van der Laan (1962) considered the adiabatic acceleration of ambient CR electrons by the radiative shock and the compressed magnetic field. The spectral index predicted by the van der Laan mechanism depends on the spectral index of low energy CR electrons with energies around $30 \mathrm{MeV}$ (Cox et al. 1999). However, spectra of low energy ambient CR electrons have not been understood completely. The radio luminosity and spectra of young supernova remnants cannot be understood by the van der Laan mechanism, and requires active acceleration of electrons by the supernova remnant shocks.

Time evolution of radio fluxes is observed in some young supernova remnants. The radio flux of Cas A has been observed for $50 \mathrm{yr}$ and decreases with time (e.g. Helmboldt and Kassim, 2009). This means that either the magnetic field or the number of CR electrons decreases with time. Not only the evolution of radio flux but also the evolution of the radio size is observed for very young supernova remnants (e.g. Weiler et al., 2002, Bartel, 2009, $\mathrm{Ng}$ et al. 2008). Therefore, we can compare evolution models of supernova remnants and observations (e.g. Martí-Vidal et al., 2011).

Polarization of radio synchrotron radiation has been also observed. Young supernova remnants have a predominantly radial magnetic field structure, whereas old supernova remnants have a circumferential magnetic field structure (Dickel and Milne, 1976; Milne, 1987). The circumferential magnetic field structure of old supernova remnants can be interpreted as the compression of the upstream magnetic field by the radiative shock. Gull (1973) suggested that the radial magnetic field of young supernova remnants results from stretch by the Rayleigh-Taylor instability. Jun and Norman (1996) confirmed that the Rayleigh-Taylor instability radially stretches the magnetic

3 Note that the power-law index of a radio spectrum $(\alpha)$ is defined for a flux energy density, whereas in X-rays one use the spectral index $(\Gamma)$ for the number density flux, the relation between the two indices is $\Gamma=\alpha+1$. If the number density of the electron distribution is labeled $q$, we have that $q=2 \alpha+1=2 \Gamma-1$. 
field. However, Schure et al. (2010) showed that the Rayleigh-Taylor instability in the gas with the adiabatic index of $5 / 3$ can not explain the radial field polarization observed near the forward shock, whereas the case of the adiabatic index of 1.1 can reproduce that. In order to make the adiabatic index of 1.1 in young supernova remnants, escaping CRs have to carry away a substantial fraction of the shock energy. Zirakashvili and Ptuskin (2008) proposed an alternative scenario that upstream density fluctuations produced by the non-resonant instability radially stretch the amplified magnetic field in the downstream region.

\subsection{X-ray observations}

Early observational evidence that supernova remnants are capable of accelerating particles to high energies mainly consisted of the radio synchrotron radiation from relativistic electrons from most supernova remnants. But the electrons responsible for the radio emission have energies in the order of a $\mathrm{GeV}$. The discovery by the $A S C A$ satellite that the featureless X-ray spectrum of SN 1006 is caused by synchrotron radiation from 10-100 TeV electrons (Koyama et al., 1995) suddenly increased the range of determined electron energies by orders of magnitude.

The X-ray emission from SN 1006 was long recognized to be peculiar, as its overall spectrum appeared to be devoid of line emission. Indeed, a synchrotron interpretation was offered by Reynolds and Chevalier (1981). But a sophisticated thermal explanation, involving fully ionized carbon-rich supernova ejecta, by Hamilton et al. (1986) was regarded as the most likely explanation. $A S C A$, however, revealed that the X-ray emission was dominated by continuum emission from near the shock front. Hot supernova ejecta are present in the interior of the supernova remnant, and produce, among others, oxygen and silicon line emission, which was hard to detect in the synchrotron dominated spectrum from the supernova remnant as a whole. The presence of oxygen-rich ejecta was clearly in contradiction to the model of Hamilton et al. (1986). Moreover, the $A S C A$ observations showed that the the continuum emission was associated with regions close to the forward shock front; an unmistakable signature of synchrotron radiation from electrons that are being actively accelerated by the supernova remnant shock.

Around the same time as the discovery of X-ray synchrotron emission from SN 1006, it became clear that some young supernova remnants emit non-thermal, hard X-ray emission. This was in particular apparent for Cas A, which is detected in hard X-rays from $\sim 10-100 \mathrm{keV}$, indicating a nearly power-law like spectrum with index $\sim 3.2$ (The et al., 1996, Allen et al., 1997; Favata et al., 1997, Vink et al. 2001; Renaud et al., 2006). For other supernova remnants hard X-ray emission up to about $30 \mathrm{keV}$ was detected (e.g. Allen et al., 1999). Although a non-thermal bremsstrahlung explanation for the hard X-ray emission of some supernova remnants exists (e.g. Asvarov et al., 1990, Favata et al., 1997; Laming, 2001; Bleeker et al., 2001), the rapid energy exchange of the $\sim 10-100 \mathrm{keV}$ electrons that cause X-ray bremsstrahlung with background electrons, makes that a power-law shaped bremsstrahlung spectrum is unlikely (Vink, 2008b). 
Since 1995, the number of X-ray synchrotron emitting supernova remnants has grown rapidly. Among the earliest identifications of X-ray synchrotron emitting supernova remnants were RX J1713.7-3946 (Koyama et al. 1997; Slane et al., 1999) and RX J0852.0-4622 (Slane et al., 2001), two supernova remnants that later turned out to be bright in $\mathrm{TeV}$ gamma-rays (Aharonian et al., 2004, 2005). Also the large diameter supernova remnant RCW 86 showed signs of non-thermal X-ray emission (Vink et al., 1997), which was later argued to be synchrotron radiation (Bamba et al. 2000 Borkowski et al. 2001).

The angular resolution of Chandra was needed to find that also the youngest supernova remnants like Tycho/SN 1572 (Hwang et al., 2001), Kepler/SN 1604 (Reynolds et al., 2007) and Cas A (Gotthelf et al., 2001, Vink and Laming, 2003) emit X-ray synchrotron emission. In those supernova remnants this non-thermal emission is concentrated in very narrow filaments $\left(1-3^{\prime \prime}\right)$ marking their shock fronts.

\subsection{Conditions for X-ray synchrotron emission}

Apart from proving that supernova remnants can accelerate particles up to at least $10^{14} \mathrm{eV}$, X-ray synchrotron emission also provides a powerful diagnostic for measuring the acceleration conditions. The presence of X-ray synchrotron filaments near shock fronts suggests that the electrons have been accelerated during the last century, or even during the last decade. This identifies supernova remnants as objects that are actively accelerating particles, rather than just objects that contain particles accelerated during an earlier phase. Moreover, as we shall see below, X-ray synchrotron emission is only possible if acceleration is surprisingly fast, with a diffusion coefficient that is rather small, and within an order of magnitude of the Bohm limit. To understand the conditions under which X-ray synchrotron emission can occur, we need a closer look at the acceleration properties in supernova remnant shocks, and at the characteristics of synchrotron emission.

Equation 11 describes the time it takes $\left(t_{\mathrm{acc}}\right)$ to accelerate a particle to an energy $E$. The maximum energy an electron (or proton) can obtain is limited by the age of the shock. For example, for a supernova remnant with $B_{-4}=0.1, V_{\mathrm{s}}=5000 \mathrm{~km} \mathrm{~s}^{-1}$ an electron (or proton) can only be accelerated in $\sim 1000$ yr to $100 \mathrm{TeV}$. If the maximum electron energy, and the corresponding synchrotron cut-off frequency, is indeed determined only by the time available for acceleration, the synchrotron spectrum is said to be age-limited (Reynolds, 1998).

However, unlike protons, electrons lose their energies quite rapidly through synchrotron radiation and inverse Compton scattering, certainly for energies in excess of $1 \mathrm{TeV}$. So another limit on the maximum energy of electrons is set by the condition $t_{\text {acc }} \approx t_{\text {loss }}$. If the maximum energy for electrons is set by this condition the synchrotron cut-off frequency is said to be loss-limited. In that case the ions can, in principle, obtain much higher energies. For magnetic fields in excess of $\sim 3 \mu \mathrm{G}$ synchrotron losses usually dominate over inverse Compton losses, unless the local radiation field is in strong excess over the microwave background radiation field. 
The synchrotron loss time for an electron is given by:

$t_{\mathrm{syn}}=\frac{E}{d E / d t}=12.5 B_{-4}^{-2}\left(\frac{E}{100 \mathrm{TeV}}\right)^{-1} \mathrm{yr}$.

The cut-off photon energy for the loss limited case can be calculated by combining Eq. 11 and Eq. 16, which gives

$E_{\max } / 100 \mathrm{TeV} \approx 0.32 \eta_{\mathrm{g}}^{-1 / 2}\left(\frac{B_{\mathrm{eff}}}{100 \mu \mathrm{G}}\right)^{-1 / 2}\left(\frac{V_{\mathrm{s}}}{5000 \mathrm{~km} \mathrm{~s}^{-1}}\right)\left(\frac{r_{4}-\frac{1}{4}}{r_{4}^{2}}\right)^{1 / 2}$,

where $r_{4}$ is the compression ratio divided by 4 . This equation can be combined with the relation between the peak in synchrotron emissivity, the so-called characteristic frequency $\nu_{\mathrm{ch}}$, for an electron with given energy $E$ moving in a magnetic field $B$ (e.g. Ginzburg and Syrovatskij, 1967):

$$
\begin{aligned}
\nu_{\mathrm{ch}} & =1.8 \times 10^{18} B_{\perp}\left(\frac{E}{1 \mathrm{erg}}\right)^{2} \mathrm{~Hz} \\
h \nu_{\mathrm{ch}} & =13.9\left(\frac{B_{\perp}}{100 \mu \mathrm{G}}\right)\left(\frac{E}{100 \mathrm{TeV}}\right)^{2} \mathrm{keV},
\end{aligned}
$$

with $B_{\perp} \approx \sqrt{2 / 3} B$ the magnetic field component perpendicular to the motion of the electron. Taken together Eq. 18 and Eq. 17 indicate that the synchrotron cut-off frequency is expected to be (c.f. Aharonian and Atoyan. 1999: Zirakashvili and Aharonian, 2007):

$h \nu_{\text {cut }- \text { off }}=1.4 \eta_{\mathrm{g}}^{-1}\left(\frac{r_{4}-\frac{1}{4}}{r_{4}^{2}}\right)\left(\frac{V_{s}}{5000 \mathrm{~km} \mathrm{~s}^{-1}}\right)^{2} \mathrm{keV}$.

This shows that in the loss-limited case the cut-off frequency does not depend on the magnetic field strength, and that for typical young supernova remnants like Cas A, Kepler and Tycho, which have $V_{\mathrm{s}} \approx 5000 \mathrm{~km} \mathrm{~s}^{-1}$, one expects to find X-ray synchrotron emission provided that $\eta_{\mathrm{g}} \sim 1$. One should realize, however, that the the synchrotron emission spectrum for a given electron energy is rather broad. As a result a rather sharp, exponential, cut-off of the electron spectrum results in a more gradual turn-over of the resulting synchrotron spectrum. Nevertheless, all observed X-ray synchrotron spectra of supernova remnants are steeper than the radio spectral indices. In X-rays the observed spectral indices are $\Gamma=2-3.5$, whereas in radio $\Gamma=\alpha+1=$ $1.4-1.8$. This indicates that in X-rays the spectra are observed near or beyond the cut-off frequency. Since for $\eta_{\mathrm{g}} \gg 10$ we would not be able to detect X-ray synchrotron emission, the detection of X-ray synchrotron has already significant consequences: it suggests that the magnetic fields near the shocks of young supernova remnants are highly turbulent. 

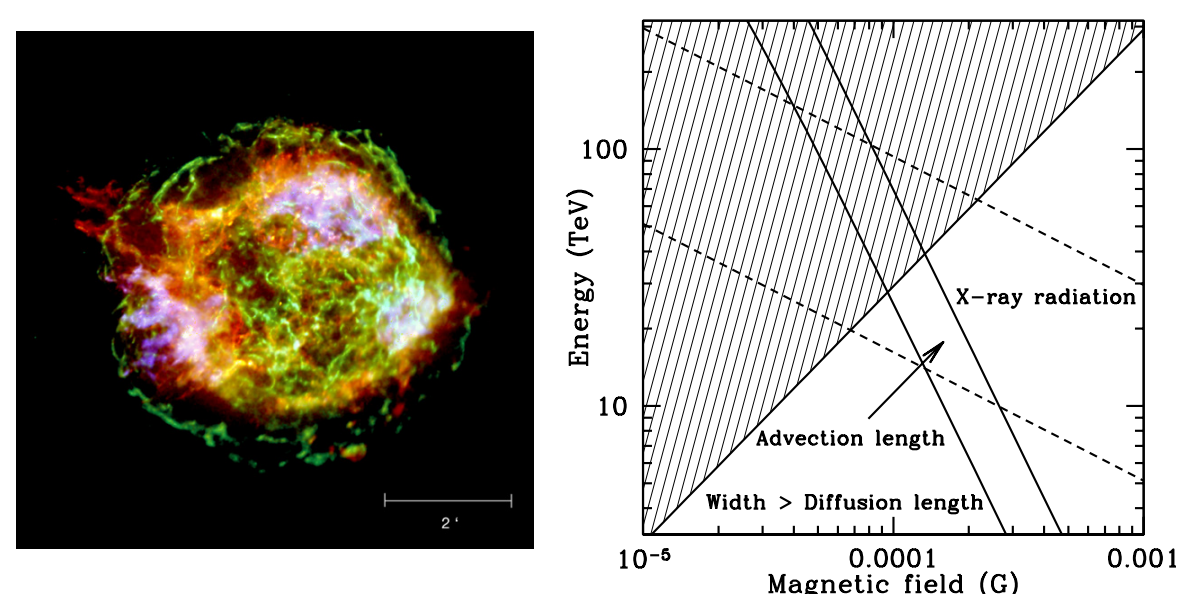

Fig. 3 Left: Chandra X-ray image of Cas A, showing in green the synchrotron dominated continuum emission. Note the narrow filaments alineating the shock front. Right: The maximum cosmic-ray electron energy versus magnetic field strength for the region just downstream of Cas A's shock front, as determined from the thickness of the filaments (Vink and Laming, 2003). The shaded area is excluded, because the filament width cannot be smaller than the minimum possible diffusion length (c.f. Vink and Laming, 2003, these figures were published before in Vink (2006)).

\subsection{The widths of X-ray synchrotron filaments}

The narrowness of the synchrotron filaments, in particular for the supernova remnants Cas A, Tycho and Kepler, is very striking. For these supernova remnants the width is typically $1-3^{\prime \prime}$, which corresponds to typical physical widths of $\sim 5 \times 10^{16} \mathrm{~cm}$. It was soon recognized that these widths indicate high magnetic fields near the shock front (Vink and Laming, 2003; Berezhko et al. 2003a; Bamba et al., 2004: Ballet, 2006). One can understand this using two different approaches. One approach is to consider that the plasma containing the highest energy electrons is moving away from the shock front with a relative velocity $\Delta v=V_{\mathrm{s}} / r$, with $r$ the shock compression ratio. As the electrons are moving outside the reach of the shock front they do not gain, but only lose energy through synchrotron radiation/inverse Compton scattering. After a certain time they have lost so much energy that they no longer emit $X$-ray synchrotron emission. The width thus corresponds to the advection length scale:

$l_{\text {adv }}=\frac{V_{\mathrm{s}} t_{\text {loss }}}{r}=4.9 \times 10^{16} r_{4}^{-1} B_{-4}^{-2}\left(\frac{V_{\mathrm{s}}}{5000 \mathrm{~km} / \mathrm{s}}\right)\left(\frac{E}{100 \mathrm{TeV}}\right)^{-1} \mathrm{~cm}$,

where we have made use of Eq. 16. In order to put a more certain limit on the magnetic field one can eliminate $E$ by using Eq. 18 to obtain:

$l_{\mathrm{adv}}=1.8 \times 10^{17} r_{4}^{-1} B_{-4}^{-3 / 2}\left(\frac{V_{\mathrm{s}}}{5000 \mathrm{~km} / \mathrm{s}}\right)\left(\frac{h \nu}{1 \mathrm{keV}}\right)^{-1 / 2} \mathrm{~cm}$.

This procedure is graphically illustrated in Fig. 3. A filament width $5 \times$ $10^{16} \mathrm{~cm}$ observed at $5 \mathrm{keV}$, therefore, corresponds to magnetic fields of 100- 
$200 \mu \mathrm{G}$, or even more if one realizes that the measured filament width overestimate the physical width due to projection effects (c.f. Berezhko et al. 2003b). This means that for Cas A and Tycho's supernova remnant the magnetic fields are typically $100-500 \mu \mathrm{G}$, well above the compressed magnetic field of the Galaxy, which would be around $15 \mu \mathrm{G}$.

The other way to estimate the magnetic field is by noting that, as a result of diffusion, a population of particles will typically spread out over a region equal to the diffusion length scale. This can be expressed as

$l_{\mathrm{diff}} \approx 2 \frac{D_{2}}{\Delta V_{\mathrm{s}}}=2 \frac{D_{2} r}{V_{\mathrm{s}}}=5.3 \times 10^{17} B_{-4}^{-1} \eta_{\mathrm{g}} r_{4}\left(\frac{V_{\mathrm{s}}}{5000 \mathrm{~km} \mathrm{~s}^{-1}}\right)^{-1}\left(\frac{E}{100 \mathrm{TeV}}\right) \mathrm{cm}$.

Here, $D_{2}$ is the diffusion coefficient behind the shock front. This puts at least a minimum to the possible width of the filaments (see the shaded area in Fig. 3). Interestingly, this methods has a difference dependence on $r, E$ and $B$. Nevertheless, using this estimate gives virtually the same magnetic field values as the advection method (see for example Ballet, 2006, Parizot et al. 2006).

In Eq. 22 one can replace $E$ with $h \nu$ using Eq. 18 (and ignoring the subtle distinction between $B_{\perp}$ and $B$, which is probably small in the post-shock region anyway):

$l_{\text {diff }} \approx 1.4 \times 10^{17} B_{-4}^{-3 / 2} \eta_{\mathrm{g}} r_{4}\left(\frac{V_{\mathrm{s}}}{5000 \mathrm{~km} \mathrm{~s}^{-1}}\right)^{-1}\left(\frac{h \nu}{1 \mathrm{keV}}\right)^{1 / 2} \mathrm{~cm}$.

This still relies on the idea that the observed photon energies $h \nu$ correspond roughly to the characteristic photon energies $h \nu_{\mathrm{ch}}$, which may not be necessarily true given the broad synchrotron emissivity function for a given electron energy. However, one can make use of another property of loss-limited synchrotron emission. Eq. 11 and Eq. 22 together give

$t_{\mathrm{acc}} \approx 2.8 \frac{l_{\mathrm{diff}}}{V_{\mathrm{s}}}\left(\frac{r_{4}}{r_{4}-\frac{1}{4}}\right)$

Combining this with the condition $t_{\text {loss }} \approx t_{\text {acc }}$ we see that

$l_{\mathrm{diff}} \approx 1.5\left(r_{4}-\frac{1}{4}\right) l_{\mathrm{adv}}$,

This assumes that synchrotron losses are dominated by the losses in the downstream region, where the magnetic field is higher than in the shock precursor (c.f. Vink, 2005, Parizot et al., 2006, Vink et al., 2006). For $r=4$ this gives $l_{\text {diff }} \approx l_{\text {adv }}$. This shows that estimating magnetic fields through the advection length scale is almost equivalent to estimating them using the diffusion length scale.

Using Eq. 20 and 22 to eliminate $E$ one finds an expression for calculating the typical downstream magnetic field that is independent of $V_{\mathrm{s}}$ and only weakly dependent on the compression ratio and $\eta_{\mathrm{g}}$. But it does rely on the 
assumption that the electrons causing the radiation have energies around the cut-off energy (e.g. Parizot et al. 2006, Vink et al., 2006)

$B_{2} \approx 26\left(\frac{l_{\mathrm{adv}}}{1.0 \times 10^{18} \mathrm{~cm}}\right)^{-2 / 3} \eta_{\mathrm{g}}^{1 / 3}\left(r_{4}-\frac{1}{4}\right)^{-1 / 3} \mu \mathrm{G}$.

The observed width $l_{\text {obs }}$ will be higher than this. First, because the observed width is the width of the projected synchrotron emitting rim, not the actual width. Second, since both advection and diffusion play a role, the synchrotron emitting shell has a width that is determined by the convolution of the advection and diffusion length scales. So roughly $l_{\mathrm{obs}} \approx \sqrt{2} l_{\mathrm{adv}}$. The projection effects depend on the ratio of the physical width to the shock radius, and on possible deviations from spherical symmetry (e.g. Berezhko et al., 2003b).

Note that Eq.26 is in principle less dependent on systematic uncertainties than either Eq. 20 and 23, because it does not depend on the measured velocity and it depends only weakly on the assumed compression ratio. But it does rely on the assumption that the emission is caused by electrons with energies close to the cut-off energy. This seems, in general, well justified for $\mathrm{X}$-ray emission from young SNRs, because these are characterized by X-ray power-law indices that are steeper than the radio spectral index $(\alpha+1)$. It is of interest to that Eq. 20 and 23 should only give similar magnetic field estimates if the assumption that electrons are close to the cut-off energy is valid (Vink, 2005, Parizot et al., 2006). The fact that indeed the magnetic field estimate based on either Eq. 20 and 23 give similar answers (Ballet, 2006) gives additional justification for the use of Eq. 26. Interestingly, this also implies that the compression ratio cannot be deviating too much from $r=4$, since Eq. 20 and 23 have a different dependence on $r$. However, if is there is a reason to expect that synchrotron radiation is observed at much lower frequencies than the cut-off frequency, Eq. 20 is to be preferred. See for example the use of Eq. 20 to determine the magnetic field properties of a radio-arc in a cluster of galaxies by van Weeren et al. (2010). 

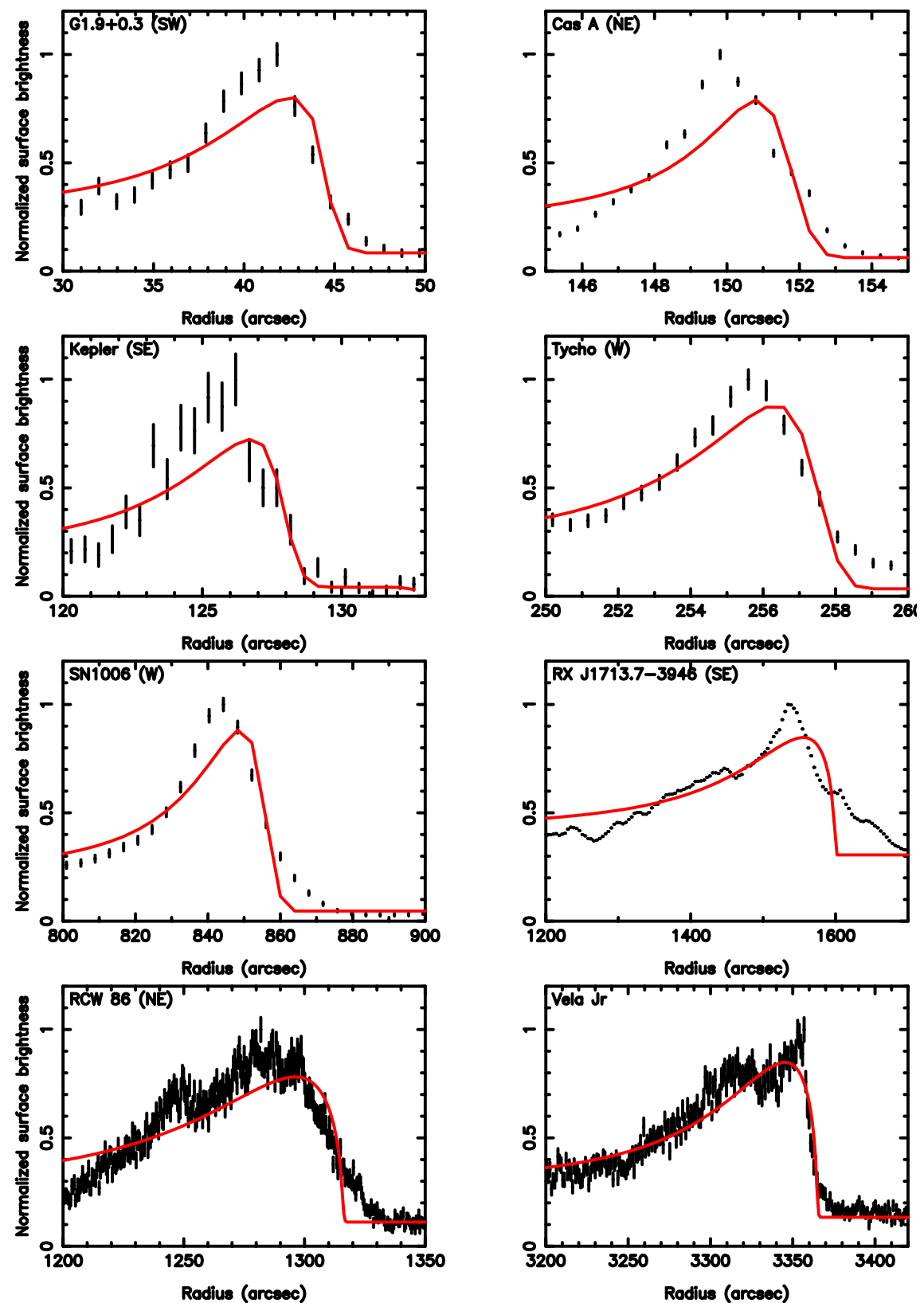

Fig. 4 Overview of X-ray synchrotron emission profiles in several supernova remnants. The profiles are extracted from Chandra observations, with the exception of RX J1713.7-3946 which is based on an XMM-Newton mosaic provided to us by Dr. Fabio Acero (Acero et al. 2009b). The solid red line indicates a best fit model based on a spherical model (i.e. the emissivity is projected onto the sky) with an exponential fall off in emissivity away from the shock front. See text for further explanation. 

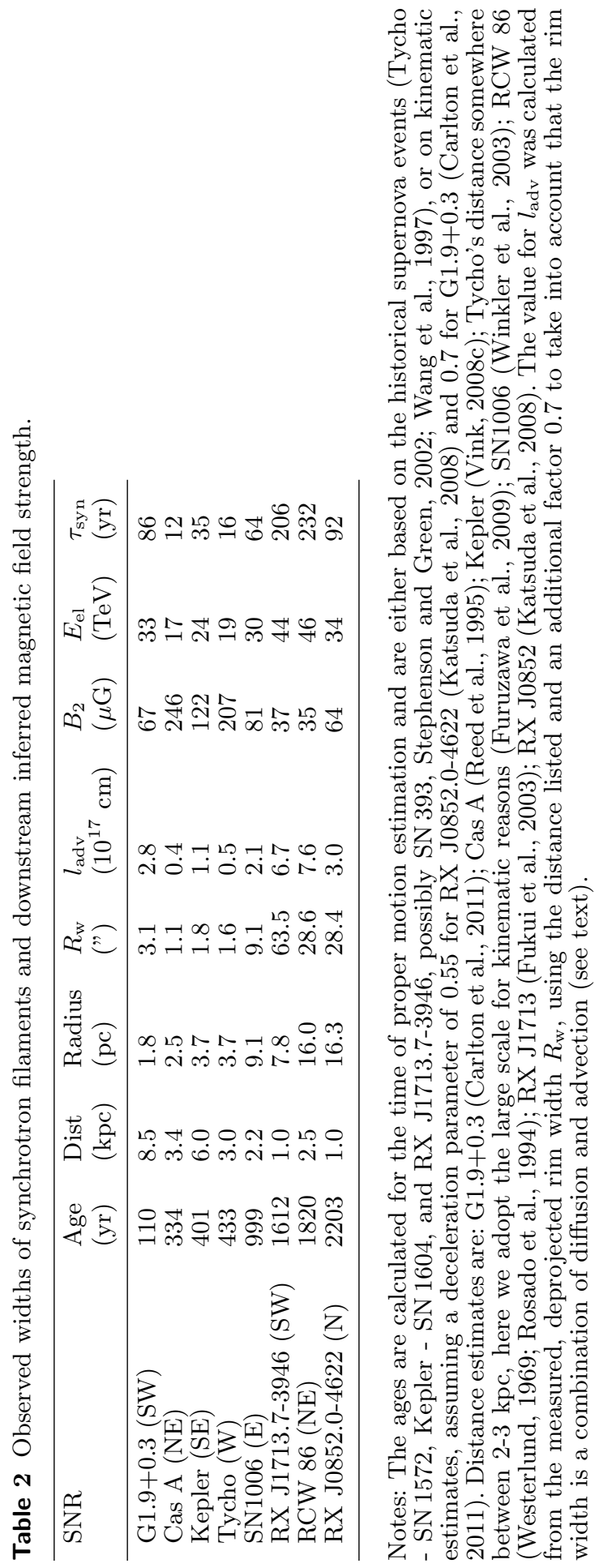


\subsection{Magnetic field measurements}

Fig. 4 shows the synchrotron emission profiles of several, relatively bright, supernova remnants. In all cases the emission is dominated by synchrotron emission, which means that for X-ray spectral line-rich supernova remnants, such as Cas A, Kepler, and Tycho, the line-poor spectral band of 4-6 keV was used. The red lines show emission models consisting of an emissivity that falls off exponentially toward the center $\left(I \propto \exp \left(-\left(R-R_{\mathrm{S}}\right) / R_{\mathrm{w}}\right), R_{\mathrm{w}}\right.$ is the width of the emitting region, c.f. Bamba et al., 2005), but, unlike Bamba et al. (2005), here spherical projection effects are taken into account. X-ray synchrotron emission from the pre-cursor is not fitted for here, because the lower magnetic field upstream of the shock should result in a much lower Xray emissivity (Berezhko et al. 2003b). Nevertheless, this issue deserves more attention in future studies, as precursor emission can be used to constrain the diffusion constant upstream of the shock (see Achterberg et al., 1994, for a study based on radio observations).

As Fig. 4 shows, the model is far from perfect ${ }^{4}$. There can be several reasons why the observed profiles are not well fitted with the exponential model. First, in some cases there are large scale deviations from spherical symmetry. A case in point is SN 1006, for which Willingale et al. (1996) and Rothenflug et al. (2004) have shown that the X-ray synchrotron emission is coming from two caps, rather than from a spherical shell. This can be taken as evidence for a preference for acceleration in magnetic field orientations that are on average parallel to the shock normal (Rothenflug et al., 2004). Indeed, Fig. 4 shows that the model is over-predicting the emission from the central region. Second, even though an exponential model may be a reasonable description in general, the acceleration of electrons to TeV energies may fluctuate with time, and also the magnetic fields is likely to vary from one region to another. For steep synchrotron spectra (all spectra here have spectral indices of $(\Gamma=2-3.5)$ a small change in magnetic field lead to large changes in emissivity $\left(I \propto B^{\Gamma}\right.$, Ginzburg and Syrovatskij, 1967). Magnetic field variations can be the result of large scale Alfvén waves, as explained in Sect 2.9. This caveat is related to the steady-state assumption that goes in deriving Eq. 20. For example, during a typical acceleration time, or synchrotron loss time, the shock velocity or the magnetic field may have substantially changed. Finally, magnetic-field amplification (see elsewhere in this volume and Sect.2.7) operates most likely in the upstream region. In the downstream region the turbulent, amplified magnetic field may decay downstream (Pohl et al. 2005). This would make in fact the magnetic estimates based on Eq. 20 an overestimate. But strong magnetic field decay would also affect the radio synchrotron morphology. This does not generally appear to be the case (e.g. Cassam-Chenaï et al., 2007), with the possible exception of one filament in the northeast of Tycho's supernova remnant (Reynolds et al. 2011). Nevertheless, some form of decay may occur in general, and would affect the geometry of the X-ray synchrotron rims.

\footnotetext{
4 Note that some regions were excluded from the fit, for example for Tycho (West) only the emission between $237^{\prime \prime}$ and $255^{\prime \prime}$ was fitted, as the emission closer to the center may also have a thermal bremsstrahlung origin.
} 
Another assumption is that the spectral cut-off frequency is determined by radiative losses, i.e. we assume a loss-limited spectrum. These broader rims imply smaller magnetic fields $(\mathrm{Eq} 26)$ and, therefore, longer synchrotron loss times. During these synchrotron loss times the shock properties may have changed, i.e. the shock velocity was likely higher in the past, and the densities may have been different in the past. Another case where this assumption may not be valid is for the very young SNR G1.9+0.3.

We note here that a projected uniform thin shell emissivity fits in many cases the observed profiles as well, or even better than an exponential emissivity model. A thin shell has a peak that lies more inward of the shock radius. Indeed, the exponential model tends to underpredict the emission around the peak. A clear exception is Vela Jr (RX J0852.0-4622), where an exponential model gives a much better fit than a uniform, projected shell. The best fit shell width of the uniform model is typically a factor two larger than the best fit characteristic width of the exponential model. Magnetic field estimates based on a uniform shell model are, therefore, $40 \%$ smaller.

Table 2 lists magnetic field estimates of synchrotron rims in several supernova remnants, based on Eq. 20 and the profiles shown in Fig. 4. In order to assess whether the spectra are loss-limited or age-limited we also list a rough estimate of the synchrotron loss times, assuming a typical photon energy of $1 \mathrm{keV}$. It shows that only for G1.9+0.3 (Reynolds, 2008, Borkowski et al., 2010 Carlton et al., 2011) it seems likely that the spectrum is age- rather than loss limited. For this reason, one should treat the magnetic field estimates as upper limits. For the RCW 86, RX J1713.7-3946, RX J0852.0-3622 and possibly SN 1006 one should still be concerned about the steady-state assumption, as the synchrotron loss times are such that the shock velocity, magnetic fields may have changed during a time $\sim t_{\mathrm{syn}}$.

The X-ray synchrotron spectra are caused by 10-100 TeV electrons that are also responsible for inverse Compton scattering background photons into $\mathrm{TeV} \gamma$-rays. There is currently a controversy about whether the $\gamma$-rays emission is dominated by these inverse Compton scattered background photons, or whether the $\gamma$-ray emission is due to the decay of neutral pions created by the collision of CR nuclei with background material (see Hinton and Hofmann, 2009, for a review). The latter would directly establish the presence of accelerated nuclei (hadronic CRs). In principle, once the average magnetic field is known one can predict, for a given X-ray synchrotron flux, the expected inverse Compton $\mathrm{TeV}$ flux.

An object that played a central role in the debate about the dominant $\gamma$ ray emission is RX J1713.7-3946. It was by many considered to be an example of a supernova remnant emitting $\gamma$-rays dominated by pion decay (Aharonian et al. 2004, Berezhko and Völk, 2008, 2010). But the lack of thermal X-ray emission could be taken as evidence for a low density inside the supernova remnant, which would argue against pion-decay dominated $\gamma$-ray emission and in favor of inverse Compton dominated emission Katz and Waxman, 2008: Ellison et al. 2010). Another argument in favor of inverse Compton dominated $\gamma$-ray emission is the close resemblance between the X-ray and $\gamma$-ray morphology (Acero et al. 2009b), which makes more sense if they are both caused by the same population of particles: electrons. Also the most 


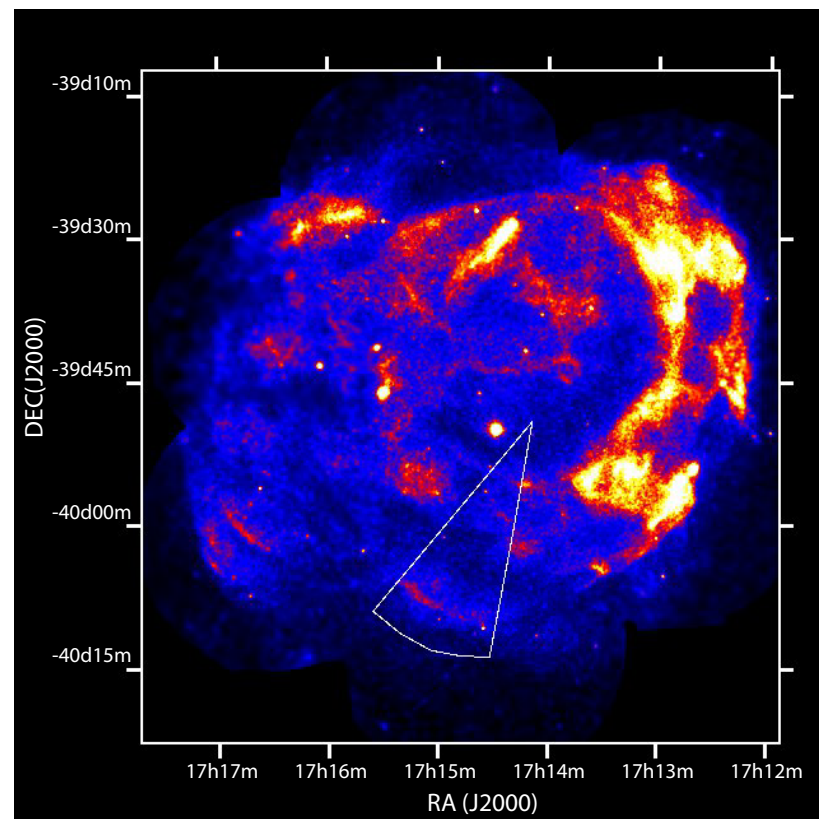

Fig. 5 The XMM-Newton X-ray mosaic of RX J1713.7-3946 kindly provided by Dr. F. Acero (Acero et al. $2009 \mathrm{~b})$. The sector indicate (thin white line) indicates the region that was taken to make the emission profile in Fig. 4

recent Fermi-LAT results, combined with TeV results, favor inverse Compton dominated emission (Abdo and Fermi LAT Collaboration, 2011). This implies that the average magnetic field must be relatively small, $10 \mu \mathrm{G}$. This seems at odds with the observed fluctuations of X-ray synchrotron filaments, which, if interpreted as corresponding to synchrotron loss times, implies magnetic field of $0.1 \mathrm{mG}$ (Uchiyama et al. 2007 , but see Sect. 2.9). Often also the size of the X-ray synchrotron filaments are used to advocate a high magnetic field inside RX J1713.7-3946. In that case one usually concentrates on bright X-ray synchrotron structures. But as the RX J1713.7-3946 panel in Fig. 4 shows, these filaments are embedded inside a broad plateau of X-ray synchrotron emission. The overall X-ray synchrotron emission comes from a very broad region. For the south-eastern region of RX J1713.47-3946 a magnetic field of $B \sim 35 \mu \mathrm{G}$ is inferred (Table 2), but several other sectors of this supernova remnant have even broader emission zones (Fig. 5). An average magnetic field of $B \sim 10 \mu \mathrm{G}$ is, therefore, quite plausible ${ }^{5}$

${ }^{5}$ There has been little attention to this issue, but see http://online.itp.ucsb. edu/online/astroplasmas_c09/vink/pdf/Vink_AstroPlasmasConf_KITP.pdf. 


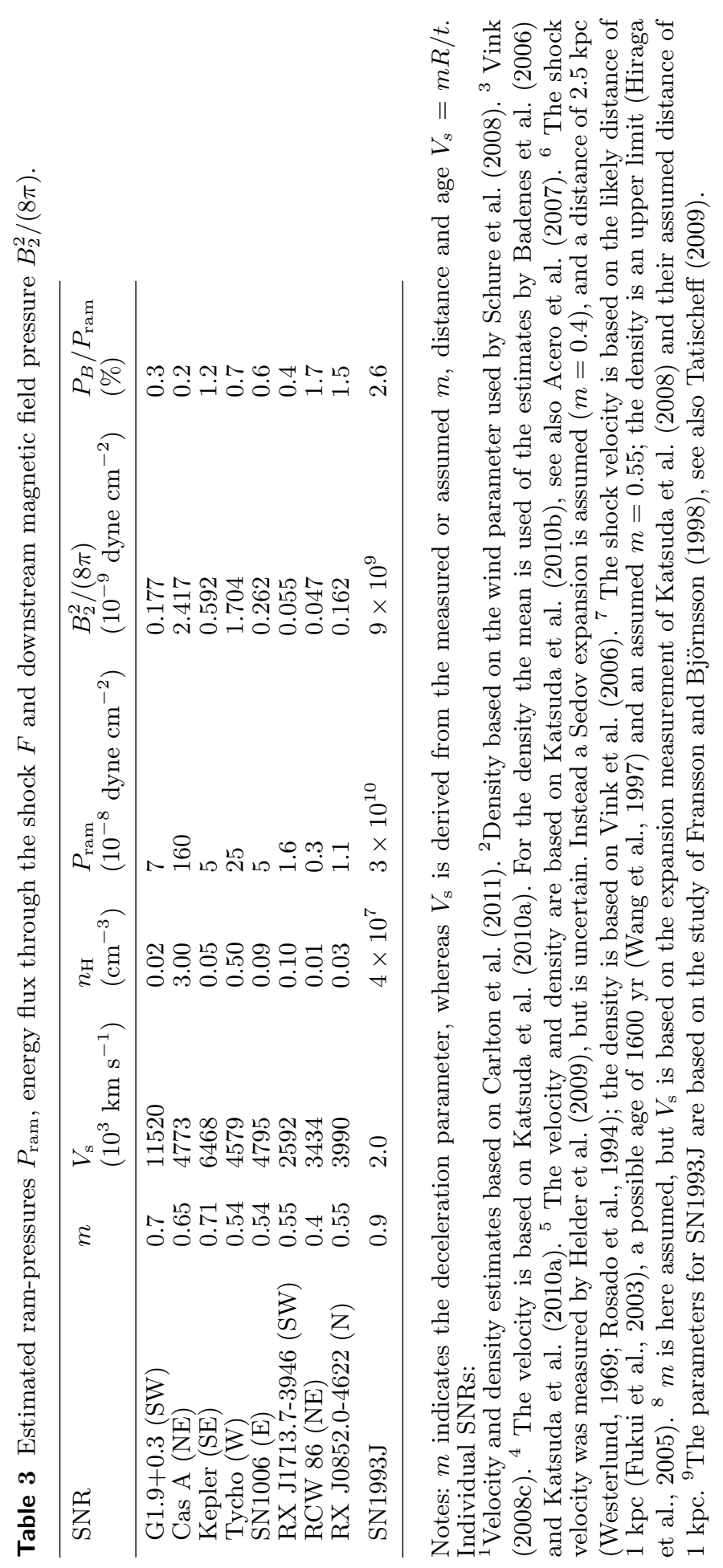




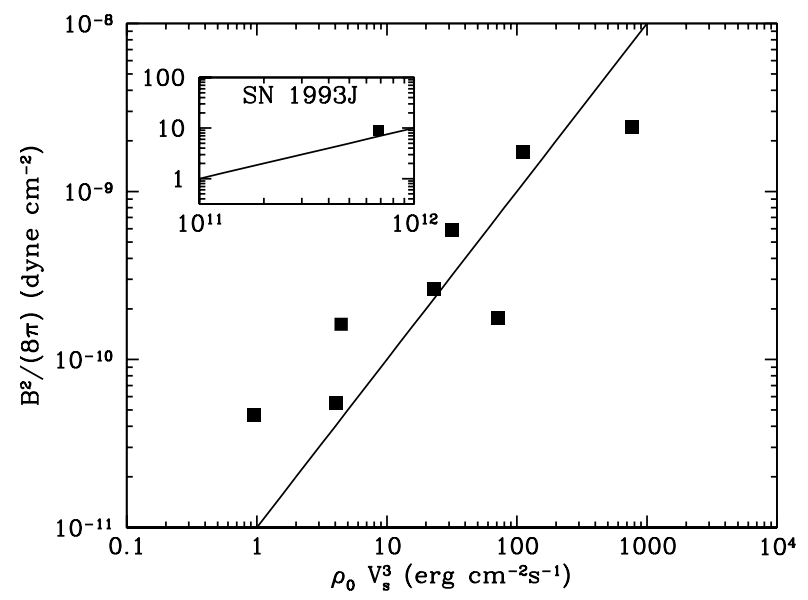

Fig. 6 The downstream magnetic field pressure versus the energy flux through the shock $\rho_{0} V_{s}^{3}$. The data points are based on the values listed in Table 3 . Note that the errors are quite large in both vertical and horizontal directions, and that the errors are largely systematic errors. The solid line indicates the relation $B^{2} /(8 \pi)=$ $10^{-11.1} \rho_{0} V_{s}^{3}$. The inset shows the values for SN1993J (Fransson and Björnsson 1998) and the solid line depicts the same relation as in the main panel.

\subsection{The case for magnetic field amplification}

The average magnetic field in the interstellar medium is of the order of $5 \mu \mathrm{G}$. After a supernova remnant shock compresses the perpendicular magnetic field component with a factor four (or more) the resulting post-shock magnetic field should be $B_{2} \sim 15 \mu \mathrm{G}$. As Table 2 shows, all supernova remnants seem to have magnetic fields higher than this; Cas A and Tycho have even considerably higher magnetic fields. This has been taken as evidence that some form of magnetic field amplification is operating in the vicinity of shocks of young supernova remnants (Vink and Laming, 2003, Berezhko et al., 2003b. Bamba et al., 2005: Ballet, 2006).

Their are several means by which this magnetic field amplification occurs (see elsewhere in this volume), but a mechanism that received a lot of attention is the so-called Bell's instability (Bell, 2004). For this mechanism the magnetic field energy density scales as $B^{2} \propto u_{\mathrm{cr}} V_{\mathrm{s}} \propto \rho_{0} V_{s}^{3}$, with $u_{\mathrm{cr}}$ the CR energy density in the CR precursor.

Völk et al. (2005) used their magnetic field determination based on the X-ray synchrotron rims to show that $B^{2} \propto \rho_{0} V_{s}^{2}$, i.e. a roughly fixed fraction of the energy density in the shocked plasma comes from the magnetic field. However, Vink (2006), using a more up to date determination of the shock velocity of Cas A and slightly different magnetic field estimates, showed that $B^{2} \propto \rho_{0} V_{\mathrm{s}}^{3}$ seems to fit somewhat better.

Fig. 6. shows the downstream magnetic energy density versus $\rho_{0} V_{\mathrm{s}}^{3}$ for the magnetic fields listed in Table 2 and densities and velocities in Table 3 . There is indeed a strong correlation. However, plotting $B^{2}$ versus $\rho_{0} V_{s}^{2}$ would give 
a roughly similar correlation. The reason is that although there is a quite a dramatic contrast among young supernova remnants in $\rho$, there is little dynamic range in $V_{\mathrm{s}}$. Moreover, there is quite some systematic uncertainty in $V_{\mathrm{s}}$, as they have not been measured for all supernova remnants, and even if measured through proper motions, there is quite some uncertainty in the distance estimates toward the supernova remnants. What makes the case for a $B^{2} \propto \rho V_{\mathrm{s}}^{3}$ relation more plausible is that it better connects to the data point corresponding to the well studied radio supernova SN 1993J (Fransson and Björnsson, 1998, Tatischeff, 2009). This radio supernova was observed to have a very high shock velocity of $20,000 \mathrm{~km} \mathrm{~s}^{-1}$, and its shock was moving through the dense wind of the progenitor. These values are dramatically different from those of young supernova remnants. However, an assumption has to be that despite these different regimes for the parameters the underlying physics for the magnetic field amplification in SN1993J is the same as for the young supernova remnants.

What is striking in Table 2 is that the lower magnetic fields are associated with larger supernova remnants (SN 1006, RX J1713.7-3946, RX J0852.04622). This can be easily understood by noting that there is probably a linear relation between $B^{2}$ and $\rho_{0}$ and that for X-ray synchrotron radiation one needs shock velocities $>2000 \mathrm{~km} \mathrm{~s}^{-1}$. Only supernova remnants in low density environments can reach radii of more than $7 \mathrm{pc}$ and still have such high velocities. But low densities does, according to our knowledge of magnetic amplification, correspond to low magnetic fields.

\subsection{X-ray synchrotron flux changes}

As a supernova remnant expands its shock velocity decelerates and the plasma and relativistic particles cool adiabatically. The synchrotron emission will, therefore, change as a function of time. This was first noticed for the radio-synchrotron emission from Cas A whose flux density changes by about 1\% per year (Högbom and Shakeshaft, 1961; Baars et al., 1977). This secular decrease was interpreted by Shklovsky (1968) as due to adiabatic losses.

For X-ray synchrotron radiation adiabatic losses may also be taken into account, but a more prominent factor is the change in shock velocity, as this changes the cut-off frequency in the loss limited case (Eq. 19), which has a strong effect on the synchrotron flux above the cut-off frequency. If we take for example the following asymptotic form of the synchrotron spectrum (c.f. Zirakashvili and Aharonian, 2007; Katsuda et al., 2010b; Patnaude et al. 2011):

$F(h \nu)=\phi B^{\Gamma_{0}} \times(h \nu)^{-\Gamma_{0}} \exp \left(-\sqrt{\frac{\nu}{\nu_{\text {cut }- \text { off }}}}\right)$,

with $\Gamma_{0}$ the asymptotic spectral index below the cut-off and $\phi$ a normalization constant, we see that the spectral index is given by:

$\Gamma=\Gamma_{0}+\frac{1}{2} \sqrt{\frac{h \nu}{h \nu_{\text {cut }- \text { off }}}}$, 


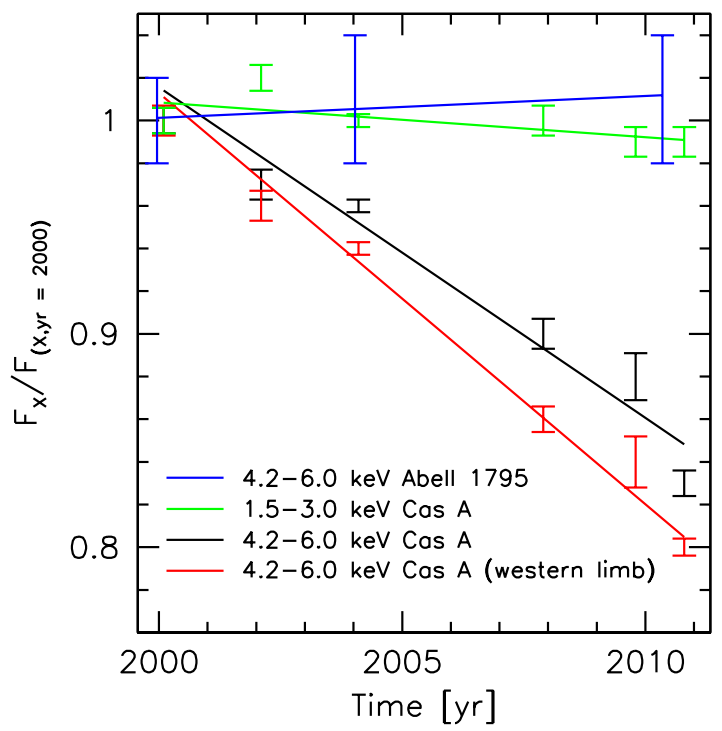

Fig. 7 The flux decrease of the SNR Cas A in the $4.2-6 \mathrm{keV}$, synchrotrondominated, X-ray band, based on Chandra observations (Patnaude et al., 2011). (Courtesy Dr. D. Patnaude, reproduced by permission of the AAS.)

with $h \nu_{\text {cut-off given by Eq. } 19}$

The fractional change in cut-off frequency as a function of the change in shock velocity is determined by:

$\frac{1}{\nu_{\text {cut }- \text { off }}} \frac{\nu_{\text {cut }- \text { off }}}{d t}=2 \frac{1}{V_{\mathrm{s}}} \frac{d V_{\mathrm{s}}}{d t}=2 \frac{(m-1)}{t}$,

with $t$ the age of the supernova remnant and $m$ the so-called expansion parameter characterizing the evolution of the supernova remnant $\left(R \propto t^{m}, V_{\mathrm{s}}=\right.$ $m R / t)$.

We can use this to estimate the expected X-ray synchrotron flux change due to changes in $h \nu_{\text {cut-off }}$ alon $\AA^{6}$

$\frac{1}{F(\nu)} \frac{d F(\nu)}{d t}=-\frac{1}{2 \sqrt{\frac{\nu}{\nu_{\text {cut }- \text { off }}}}} \frac{\nu}{\nu_{\text {cut }- \text { off }}^{2}} \frac{d \nu_{\text {cut }- \text { off }}}{d t}=\sqrt{\frac{\nu}{\nu_{\text {cut }- \text { off }}}} \frac{(m-1)}{t}$

A recent analysis of the Chandra observations of Cas A showed that this supernova remnant has a decline in X-ray synchrotron flux at a rate of $1.5 \%-2 \% \mathrm{yr}^{-1}$ (Patnaude et al., 2011, see Fig. 7). In fact, if one takes $m=0.65$ (Vink et al., 1998; Delaney and Rudnick, 2003: Patnaude and Fesen,

\footnotetext{
6 Note that Katsuda et al. (2010b) uses a similar formalism, but with slightly different dependencies as they also consider the change in the amplified magnetic field
} 
2009 ), $t=330 \mathrm{yr}$, and $\sqrt{\nu / \nu_{\text {cut }- \text { off }}} \approx 0.6-7$ (corresponding to $h \nu_{\text {cut }- \text { off }}=$ $0.1-2 \mathrm{keV}$ ), then Eq. 30 predicts a fractional decline rate of $0.06-0.7 \% \mathrm{yr}^{-1}$. This is smaller than observed, but it should be noted that the strongest decline rate seems to be associated with the X-ray synchrotron emission from the reverse shock (Sect 2.12) for which $m$ is not well measured. We also note that the cut-off frequency does not seem to be well defined. Indeed, one of the peculiar features of Cas A its near power-law spectral shape from $\sim 5-100 \mathrm{keV}$ (Renaud et al. 2006), whereas Eq 27 predicts a gradual steepening.

Eq. 28 can be used to couple the flux decline rate with a steepening of the synchrotron spectrum (Patnaude et al. 2011):

$\frac{1}{F(\nu)} \frac{d F(\nu)}{d t}=-2 \frac{d \Gamma}{d t}$

The Chandra observations of Cas A indicate $d \Gamma / d t=0.022 \mathrm{yr}^{-1}$, which should correspond with a flux decline of $\sim 4 \% \mathrm{yr}^{-1}$, which is clearly too large. These discrepancies could either point to the invalidity of the simple model for flux decline (see also the discussion in Patnaude et al., 2011), or the invalidity of Eq27 to describe the overall X-ray synchrotron spectrum of Cas A.

For the youngest known supernova remnant G1.9+0.3 the X-ray synchrotron flux is measured to be increasing with a rate of $1.7 \pm 1.0 \% \mathrm{yr}^{-1}$ (Carlton et al., 2011). A similar flux increase is observed in the radio. This flux increase is most likely due to an expansion in a uniform medium. Due to an increase of the swept up mass the emission will increase, although not linearly with the swept-up mass due to adiabatic losses (Carlton et al., 2011). The same is true for the X-ray synchrotron emission, but here, like for Cas A, the change in cut-off frequency will be a large effect. If the synchrotron spectrum is age limited the change in cut-off frequency can be found by combining Eq. 11 and Eq. 18, which gives (Katsuda et al., 2010b):

$\nu_{\text {cut-off }} \propto B^{3} V_{\mathrm{s}}^{4} t^{2}$,

which, if $B$ is assumed constant, gives:

$\frac{1}{\nu_{\text {cut }- \text { off }}} \frac{d \nu_{\text {cut }- \text { off }}}{d t}=\frac{4(m-1)+2}{t}$.

This implies that the cut-off frequency will decrease for $m<0.5$, and consequently the X-ray synchrotron flux would be more likely to decrease. Note that Katsuda et al. (2010b) and Carlton et al. (2011) include a dependence of $B^{2} \propto V_{\mathrm{s}}^{2}$ and find

$\frac{1}{\nu_{\text {cut }- \text { off }}} \frac{d \nu_{\text {cut }- \text { off }}}{d t}=\frac{(7(m-1)+2)}{t}$,

with a decline in cut-off frequency for $m<0.71$. For $B^{2} \propto V_{\mathrm{s}}^{3}$ we find

$\frac{1}{\nu_{\text {cut }- \text { off }}} \frac{d \nu_{\text {cut }- \text { off }}}{d t}=\frac{(17(m-1) / 2+2)}{t}$, 
which gives a decline in cut-off frequency for $m<0.76$. These expressions can be used together with the second part of Eq. 30 to estimate flux changes in age limited cases. Note that the value for $m$ is not known for G1.9+0.3, because it is usually estimated from the relation $V_{\mathrm{s}}=m R / t$, but since G1.9+0.3 is not connected to an historical event, $t$ is not known.

The equations 33-35 show a peculiarity of the X-ray synchrotron emission for age limited spectra. If the $m$ is too small, the cut-off frequency only decreases. For the constant magnetic field case this is not so dramatic because young supernova remnants in Table 3 have in most cases $m>0.5$. But in the case of magnetic-field amplification it may in some cases be difficult to understand why there is X-ray synchrotron radiation at all. For example, for Cas A, $m=0.65$. This value corresponds roughly with a Sedov model for a supernova remnant evolving inside the wind of the progenitor. Since Cas A evolves in a dense wind $m=0.65$ is probably valid for the last $200 \mathrm{yr}$ (van Veelen et al. 2009, Fig. 7). If that is indeed the case then most of the electrons responsible for X-ray synchrotron radiation may have been electrons that were injected in the early supernova remnant phase. Indeed, in the early phase one expects $m>0.8$. For example, SN1993J was observed to have $m \approx 0.84$ (Marcaide et al. 2009).

For Type Ia supernova remnants a similar problem exists if one takes them to evolve in their early phase in a self-similar way. One would then expect $m=0.57$ (Chevalier, 1982a). But it seems more likely that the ejecta structure has an exponential profile (e.g. Dwarkadas and Chevalier, 1998), in which case in the early phase one expects $m>0.7$.

One should note, however, that magnetic-field amplification is not an instantaneous process. It probably evolves simultaneously with the acceleration of particles to higher and higher energies. This, nevertheless, shows that observations of X-ray synchrotron flux decline serve to cast a new light on the theory of the evolution of supernova remnants, the theory of X-ray synchrotron radiation, and even on the issue of magnetic field amplification.

\subsection{X-ray surface brightness variations in supernova shells}

Clumpy structures in X-ray emission are frequently observed in imaging observations of supernova remnants. These structures have X-ray spectra that can be both thermal or non-thermal in nature. Structures with thermal Xray spectra may be caused either by instabilities at the contact discontinuity, by clumps of ejecta protruding beyond the blast wave or by reverse shock heating of clumpy ejecta material. The spatial and temporal characteristics of structures with non-thermal X-ray spectra are probably caused by particle acceleration and the associated processes of fluctuating magnetic field amplification.

Here, we limit ourselves to a discussion of X-ray brightness variations in supernova shells related to energetic particle acceleration processes and therefore to the structures with non-thermal X-ray spectra. These X-ray brightness variations may originate both from the bremsstrahlung emission of electrons with keV-MeV energies and from synchrotron radiation of electrons with $\mathrm{TeV}$ energies in stochastic magnetic fields. 
2.10 Synchrotron surface brightness fluctuations and stripes in Tycho

X-ray synchrotron emission structures have been observed with the superb spatial resolution of the Chandra telescope in many young supernova remnants (see e.g., Vink and Laming, 2003, Bamba et al., 2005, Uchiyama et al., 2007: Patnaude and Fesen, 2009: Helder and Vink, 2008: Eriksen et al., 2011). The morphology of the extended, nonthermal, thin filaments observed at the supernova remnant edges, and their X-ray brightness profiles, strongly support the interpretation that $\gtrsim 10 \mathrm{TeV}$ electrons are accelerated at the forward shock of the expanding supernova shell and produce synchrotron radiation in an amplified magnetic field (see e.g. Reynolds, 2008, Vink, 2008a).

The small scale X-ray structures brightening and decaying on a one-year timescale were reported by Uchiyama et al. (2007) in the shells of the supernova remnant RX J1713.7-3946 and in Cas A by (Patnaude and Fesen, 2007; Uchiyama and Aharonian, 2008, Patnaude and Fesen, 2009). The rapid variability of the structures detected with Chandra were interpreted as the $\mathrm{X}$-ray synchrotron structures that are produced by ultrarelativistic electrons accelerated in a strongly magnetized environment with the magnetic field amplification by a factor of more than 100. Moreover, Uchiyama et al. (2007) suggested that the X-ray variability is a direct signature of the ongoing shock-acceleration of electrons in real time. However, the presence of the very high magnetic fields in the shell of supernova remnant RX J1713.7-3946 was shown by Butt et al. (2008) to be too constraining from the view of the multi-wavelength observations, unless the high field regions occupy a small fraction of the volume.

Efficient diffusive shock acceleration of high-energy particles requires a substantial amplification of magnetic field fluctuations in the vicinity of the shock; see e.g. Bell (1978); Blandford and Eichler (1987); Malkov and Drury (2001). Magnetic-field amplification mechanisms due to CR instabilities in nonlinear diffusive shock acceleration were proposed recently by several studies Bell (2004, 2005); Amato and Blasi (2006); Vladimirov et al. (2006); Marcowith et al. (2006); Vladimirov et al. (2009); Zirakashvili and Ptuskin (2008); Bykov et al. (2011b); Schure and Bell (2011). These models predict cosmic-ray streaming ahead of the shock will amplify the local magnetic field substantially.

An alternative explanation for the surface brightness fluctuations in RX J1713.7-3946 and Cassiopeia A was offered by Bykov et al. (2008b). As the amplified magnetic fields in supernova shells are highly turbulent, they might produce local enhancements, resulting in isolated structures in a synchrotron image that show large variations in the brightness. Note that these brightness variations can occur even if the particle distribution is smooth and steady.

Bykov et al. (2008b, 2009) investigated this effect, resulting in the following three predictions. Firstly, the cut-off energy of the emitted synchrotron spectrum can be significantly increased for a distribution of electrons residing in a turbulent magnetic field, as compared to electrons in a uniform field with the same strength. Secondly, the variation in surface brightness is on shorter timescales for images obtained at higher energies. More specifically, the time scales expected are similar to those found in X-ray images of RX J1713.7- 
3946 and Cassiopeia A. Future hard X-ray imaging instruments as NuSTAR and ASTRO-H will probably be able to test this prediction. Thirdly, the model predicts that the synchrotron emission from these bright structures will be highly polarized, as illustrated in Figure 8

Recently, very unusual structures consisting of ordered sets of bright, non-thermal stripes were discovered a deep Chandra exposure of Tycho's supernova remnant (Eriksen et al. 2011). The stripes are clearly seen in the 4.0-6.0 keV image of the remnant (Fig. 9). At these energies, the emission is dominated by X-ray synchrotron radiation. Understanding these structures presents a unique opportunity for current models of X-ray synchrotron images of young supernova remnants. As the stripes are likely caused by peaks in magnetic turbulence of a perpendicular shock, this provides a way to measure the orientation of the magnetic field of the ambient medium (Bykov et al. 2011a).

Also, the coherent nature of the X-ray stripes likely suggests that the underlying magnetic turbulence is strongly anisotropic relative to an ordered mean background magnetic field. Bykov et al. (2011a) modeled Tycho's stripes assuming they are the result of the nonlinear evolution of the anisotropic CR-driven magnetic instability by (Bell, 2004, Vladimirov et al. 2009: Bykov et al., 2011b), and concluded that the structures should only occur in the shell section where the local field lies mainly along the shock surface and where the turbulence cascading is suppressed. The simulated stripes are shown in Fig. 10. They found that the orientation of the ambient magnetic field at the location of the stripes has to be quasi-perpendicular to the shock normal. The unstable growing magnetic modes must maintain coherence over a scale, close to the size of the CR precursor. Additionally, the modes have to be linearly polarized. Turbulence cascading along the mean field should be suppressed to prevent the broadening in wavenumber $(k)$ of the generated turbulence. Vladimirov et al. (2009) demonstrated in their nonlinear diffusive shock acceleration model that the spectral cascade suppression results in a peaked structure of the magnetic fluctuation spectra. The predicted polarized fraction of $\sim 50 \%$ would make these stripes easily detectable in future X-ray polarization observations

\subsection{The asymptotic behavior of particle spectra}

According to non-linear diffusive shock acceleration theory the spectrum of accelerated particles should not be a power law, but instead gradually harden. The reason is that the presence of accelerated particle ahead of the shock creates a shock precursor, which pre-compresses the gas, before it is further compressed by the gas shock. Also the overall shock compression can be substantially higher than the canonical factor four, for a high Mach number shock in a monatomic gas. At the same time, a high compression in the precursor, means a lower Mach number at the actual gas shock, as the medium has already been adiabatically heated. All this means that the highest energy particles may scatter back and forth across the total shock region, sampling the full compression ratio, whereas the low energy cosmic rays only sample the gas-shock region, which may have a compression ratio of about $r=2.5$. 

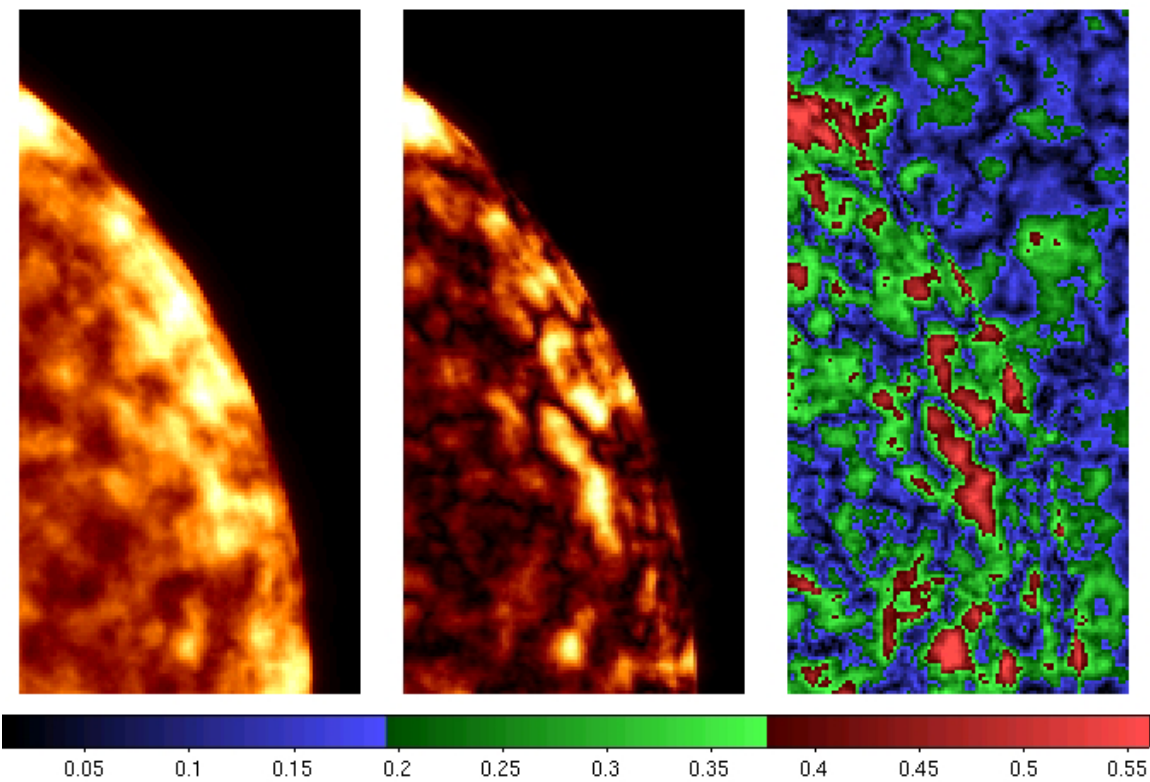

0.05

0.1

0.15

0.2

0.25

0.3

0.4

Fig. 8 Simulations of polarized synchrotron emission in a random magnetic field at $5 \mathrm{keV}$ adapted from Bykov et al. (2009). The left panel shows intensity. The central panel shows the product of intensity and polarization degree. The right panel shows the degree of polarization (colorbar represents its scale). The $\sqrt{\left\langle B^{2}\right\rangle}=3 \times 10^{-5}$ $\mathrm{G}$ and the spectral energy density of magnetic fluctuations $W(k) \propto k^{-2}$. For the models with $W(k) \propto k^{-1}$ see Figs. 2-4 in Bykov et al. (2009).

Since in the test particle approach the power-law index of the spectrum of accelerated particle is $q=(r+2) /(r-1)$ one sees that for $r=2.5 q=3$, whereas for $r=7$ it is $q=1.5$. For non-linear shock acceleration one expects a spectrum that gradually changes in spectral slope as a function of energy. For low energies the slope of the particle spectrum will be $q \approx 3$, but it is expected to asymptotically harden to $q=1.5$ at high energies if compression ratios are $r \approx 7$. Malkov (1997) showed that for even higher compression ratios one expects $q=1.5$. On the other hand, if Alfvén waves are on average directed away from the shock front a softer spectrum may be expected (Zirakashvili and Ptuskin, 2008).

Observationally, there is clear evidence that synchrotron spectra from young SNRs are not power laws over a long range of frequencies. The first evidence for this was presented by Reynolds and Ellison (1992), who showed that the radio spectra of Tycho and Kepler gradually harden from $\sim 10^{8} \mathrm{~Hz}$ to $\sim 10^{10} \mathrm{~Hz}$.

Cas A has a rather steep radio spectrum, $\alpha=0.78$, corresponding to $q=2.6$ and, on face value, corresponding to a compression ratio of $r=2.9$, with little evidence for intrinsic spectral hardening within the radio spectrum. But comparing the radio data with synchrotron emitting regions detected 


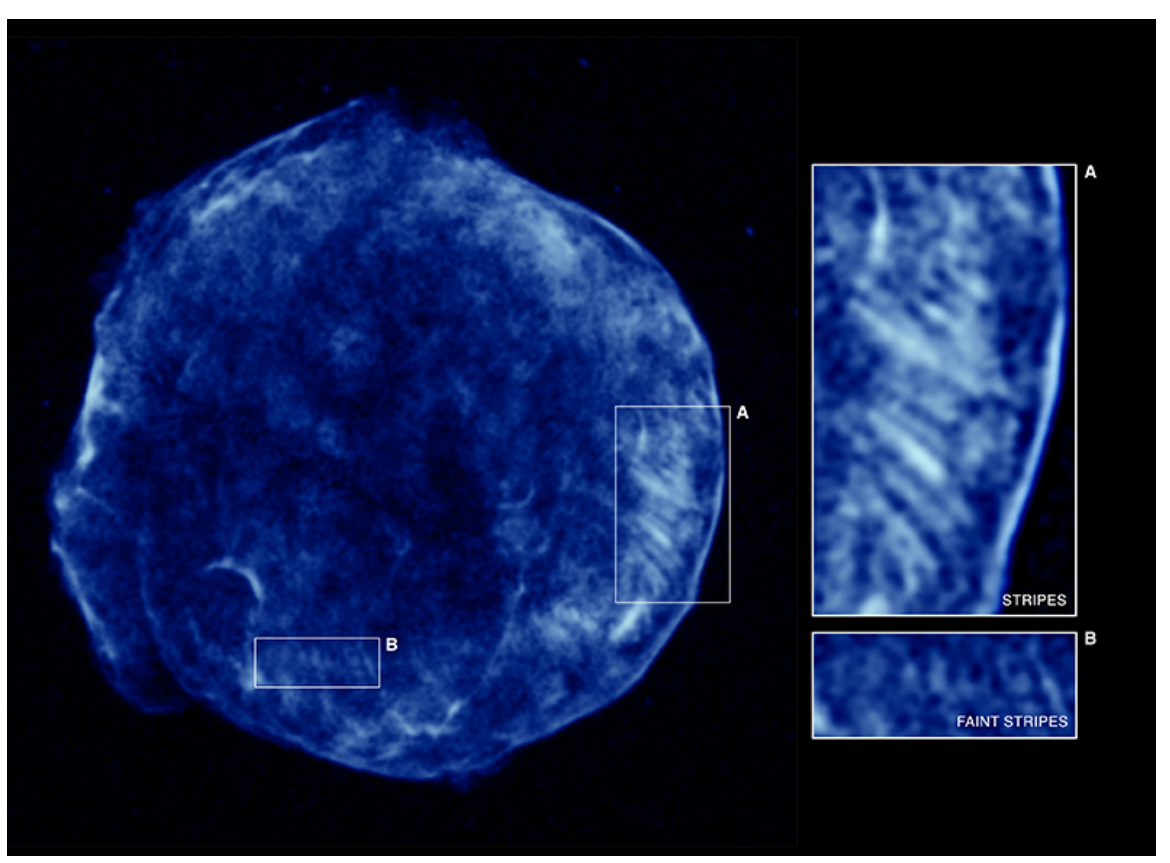

Fig. 9 Chandra X-ray 4.0-6.0 keV image of the Tycho supernova remnant, smoothed with $\mathrm{a} \sim 0^{\prime \prime} .75$ Gaussian. Adapted from Eriksen et al. (2011), courtesy to Dr. Eriksen.

in the infrared reveals that the synchrotron spectrum must have hardened (Jones et al., 2003).

Also some X-ray synchrotron spectra show that spectral hardening must occur. In X-rays the synchrotron emission is observed near or beyond the spectral cut-off frequency, but for RCW 86 (Vink et al., 2006) and SN 1006 (Allen et al. 2008) the extrapolation of the radio synchrotron spectrum cannot at the same time explain the X-ray flux and the X-ray spectral index. For both cases a curved synchrotron spectrum describes the combined radio and X-ray observations better. Note that in both cases an heuristic curved electron spectrum was chosen. In Fig. 11 the data points and curved synchrotron models are shown for the northeastern region of RCW 86. Both models with an asymptotic power-law index for the electron distribution of $q=2$ (the classic Fermi acceleration index) and $q=1.5$ can in principle explain the observations. Note that incorporating curvature in the models will lead to lower values for the cut-off frequency when compared to a classic, but very much simplified, X-ray synchrotron model as srcut (Reynolds and Keohane. 1999). Note that one should take into account that the radio emission from a SNR is likely to come from a much larger volume than the X-ray synchrotron emission. So it is better to apply this type of analysis to small X-ray emitting regions, rather than to emission from the entire SNR.

Although the evidence for curvature is quite compelling, we see that the evidence for an asymptotic particle power-law index of $q=1.5$, expected for 


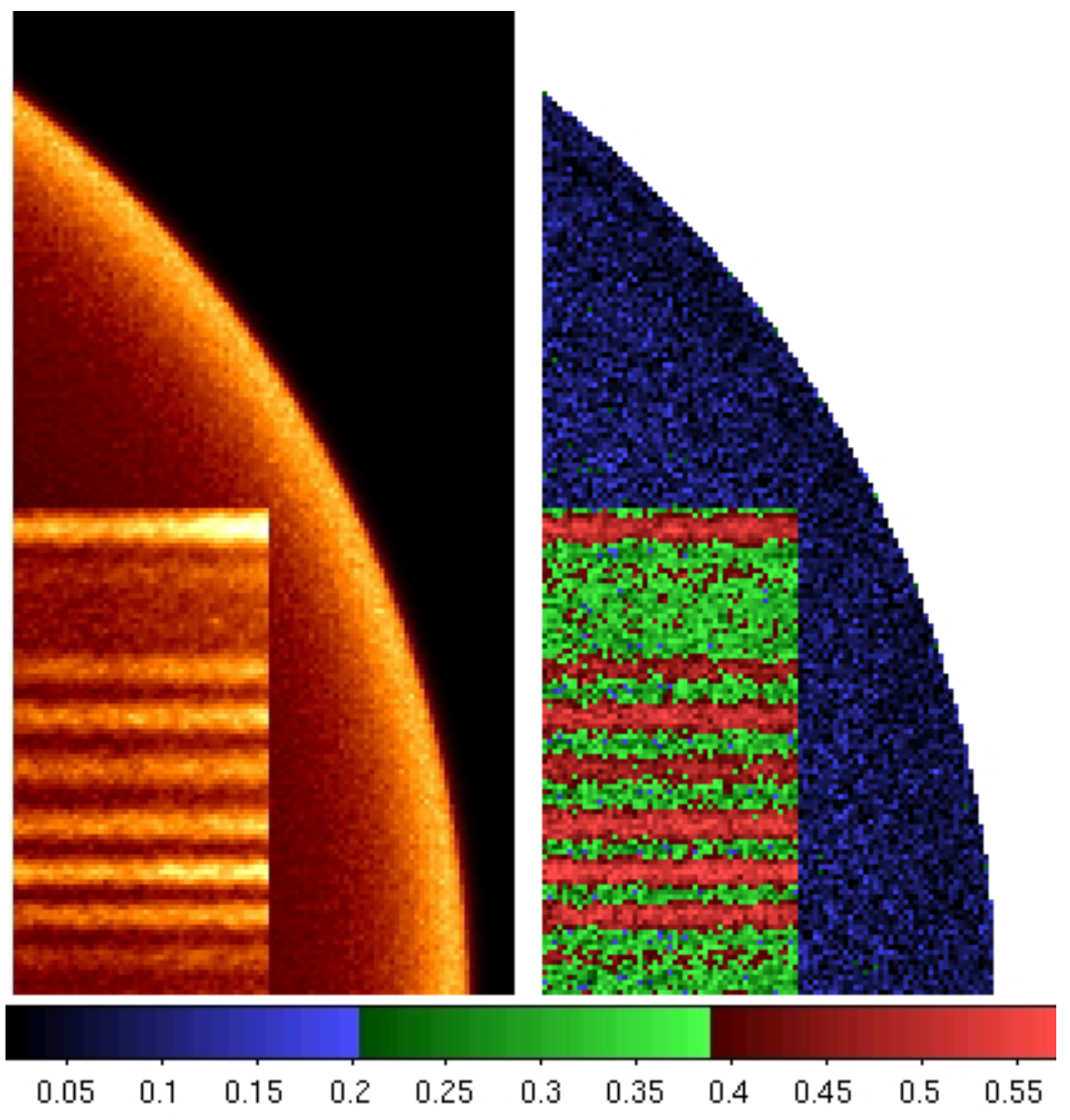

Fig. 10 Supernova remnant synchrotron emission images simulated by Bykov et al. (2011a) to model the Tycho supernova remnant stripes. Simulations done within a trame of the nonlinear model of diffusive shock acceleration taking into account magnetic field amplification from a CR current driven instability. The left panel is the synchrotron X-ray intensity at $5 \mathrm{keV}$ regime. The degree of polarization of the $\mathrm{X}$-ray emission is in the right panel with the degree of linear polarization shown in the color bar. The high degree of polarization of the X-ray synchrotron stripes is evident. Reproduced by permission of the AAS.

strongly non-linear acceleration, is much less clear. Also $\mathrm{TeV} \gamma$-ray observations do not provide evidence for spectra that are that hard, with most observed spectral indices $\Gamma>2$. But the measurements errors are still large enough, and the interpretation of the results (pion-decay or inverse Compton scattering) still uncertain enough, that one cannot yet firmly rule out $q=1.5$. 


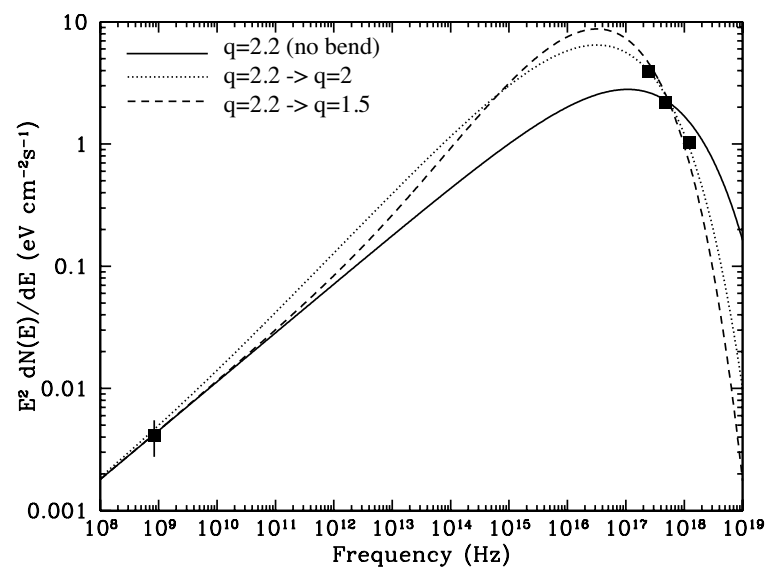

Fig. 11 Broad band energy density spectrum of the northeastern part of RCW 86. The radio spectrum, here represented with a flux density point at $1 \mathrm{GHz}$ and a spectral index $\alpha=0.6$, cannot be connected with the X-ray flux point if one assumes an electron spectrum consisting of a power law with an exponential cut-off. Instead a gradual flattening is needed, although there is enough freedom to either have a modest flattening, with an asymptotic index of $q=2$ or to have $q=1.5$ predicted by non-linear shock acceleration theory. (Figure from Vink et al., 2006, reproduced by permission of the AAS.).

\subsection{Particle acceleration at the reverse shock}

Throughout the Sedov phase of the supernova remnant evolution, the shock velocity of the reverse shock can be comparable to, or even higher than, the forward shock velocity (Truelove and McKee, 1999). This in itself makes the reverse shock a probable location for CR acceleration. However, in contrast to the magnetic field of the ambient medium, the magnetic field in the freely expanding ejecta (which is upstream for the reverse shock) is negligible. As magnetic fields are essential for particle acceleration processes, it is not obvious that reverse shocks are able to accelerate particles (Ellison et al., 2005). Observational evidence for cosmic-ray acceleration at the reverse shock would provide useful constrains on the required magnetic field upstream to make particle acceleration work.

A number of observations suggest synchrotron emission from the reverse shock. DeLaney et al. (2002) found radio synchrotron emission coinciding with the reverse shock, implying the presence of $\mathrm{GeV}$ electrons. Also, the southwest corner of the RCW 86 remnant shows signs of X-ray synchrotron emission. This corner is interacting with a cavity wall and as a reaction, the reverse shock probably developed very strongly in this part of the remnant. Coincidentally, it is exactly in this part of the remnant where there are distinct filaments of non-thermal X-ray emission inside of the thermal emission at the outside (Rho et al. 2002), suggesting the presence of accelerated particles at the reverse shock. Additionally, Zirakashvili and Aharonian (2010) 
suggested that the arc-like structure in the X-ray synchrotron emission of RX J1713.7-3946 is formed by the reverse shock as well (See Figure 5).

The non-thermal X-ray emission of Cas A is rather complex. The origin of the narrow filaments that mark the outside of the remnant is at the forward shock, but there is non-thermal emission at the inside as well (Gotthelf et al. 2001). Helder and Vink (2008) concluded from a deprojection of the megasecond Chandra image in the 4 to $6 \mathrm{keV}$ band and the different kinematical properties (DeLaney et al. 2004) that these inner non-thermal $\mathrm{X}$-ray filaments are originating from a shell inside of the forward shock, rather than located at the forward shock, projected onto the center of the remnant. Uchiyama and Aharonian (2008) reached a similar conclusion for Cas A, based on the fact that the inner filaments showed surface brightness fluctuations and the outside structure did not (but, see Patnaude and Fesen, 2009). A non-thermal bremsstrahlung is unlikely for these inner filaments, as nonthermal tails to the electron distribution would have disappeared through Coulomb interactions on timescales shorter than the lifetime of the remnant (Vink, 2008b). Therefore, Helder and Vink (2008) concluded that the nonthermal X-ray emission at the center of Cas A is synchrotron radiation of $\mathrm{TeV}$ electrons at the reverse shock. Whether these electrons were accelerated or reaccelerated (Schure et al., 2010) at the reverse shock remains unclear.

\subsection{Gamma-ray observations of supernova remnants}

Ever since the detection of $\gamma$-rays from the Crab nebula by the Whipple Telescope (Weekes et al. 1989) showed the potential of Cherenkov telescopes, the field of TeV $\gamma$-ray astronomy is thought to hold the key to solve the mystery of the origin of Galactic CRs. But despite the tremendous advances in $\mathrm{TeV}$ $\gamma$-ray astronomy, there is, as we will discuss below, no definitive proof that the CRs are accelerated by supernova remnants in sufficient quantities and to energies beyond the knee in order to explain the CR spectrum on earth. Nevertheless, our understanding of CR acceleration in supernova remnants has increased substantially as a result of $\gamma$-ray astronomy. The results of TeV $\gamma$-ray telescopes $(\sim 0.1-100 \mathrm{TeV})$ has recently been complemented by observations with the $\mathrm{GeV} \gamma$-ray satellites $(\sim 0.1-100 \mathrm{GeV})$ AGILE (Tavani et al., 2008) and Fermi (Atwood et al., 2009).

\subsubsection{Hadronic versus leptonic $\gamma$-ray radiation}

$\gamma$-ray astronomy is of great importance for determining the CR content of astrophysical sources, because only in $\gamma$-rays one can observe photons emitted as a result of hadronic CRs (i.e. CR nuclei/ions), which make up 99\% of the CRs observed on earth. The emission is caused by CR nuclei colliding with the background medium, thereby producing, among others, pions (pimesons). The neutral pions decay immediately into two photons, each having in the rest-frame of the collision an energy of half that of the pion rest mass, $M_{\pi^{0}} c^{2}=135.0 \mathrm{MeV}$. In the frame of the observer the photon has an energy that is on average about $12 \%$ of the energy of the primary CR particle. The 
$\gamma$-ray spectrum and emissivity is directly related to the spectrum of CRs in the source and the density of the background medium, $n_{\mathrm{H}}$. If the primary particle spectrum is given by

$n_{\mathrm{cr}}\left(E_{\mathrm{p}}\right) d E_{\mathrm{p}}=k E_{\mathrm{p}}^{-q_{\mathrm{p}}} d E_{\mathrm{p}}$,

with $k$ a normalization constant and $q_{\mathrm{p}}$ the spectral index, the $\gamma$-ray emissivity is given by

$\frac{d n(h \nu)}{d h \nu d t}=n_{\mathrm{H}} \int c k_{\mathrm{p}} E_{\mathrm{p}}^{-\mathrm{q}_{\mathrm{p}}} \sigma_{\mathrm{pp}, \text { inel }}\left(E_{\mathrm{p}}\right) \frac{d f\left(h \nu, E_{\mathrm{P}}\right)}{d h \nu} d E_{\mathrm{p}}$.

Here $c$ is the velocity of the $\mathrm{CR}, \sigma_{\mathrm{pp} \text {,inel }}$ the inelastic collision cross section, and the function $d f\left(h \nu, E_{\mathrm{p}}\right) d E_{\mathrm{p}} / d h \nu$ gives the energy distribution of the $\gamma$-rays as a function of the primary proton energy. This function includes the effects of multiplicity $\left.\right|^{7}$ (the number of pions produced per collision), the distribution of energies of the pions, and emission angle dependence. In addition, it should include the effects of composition of both the cosmic rays and the background plasma. An approximation that is sometimes made is that for a large range of energies $\sigma_{\mathrm{pp} \text {,inel }}$ is assumed to be constant, and $<h \nu / E_{\mathrm{p}}>\approx 0.17$ (including the contribution of $\eta$-meson production, Aharonian and Atoyan, 2000). Since the pion production cross section is only mildly energy dependent (see Kelner et al., 2006, for approximate formulae), the spectral photon index is approximately that of the spectral index of the particles, $\Gamma \approx q_{\mathrm{p}}$.

However, the detection of $\gamma$-rays cannot be uniquely attributed to CR nuclei, because relativistic electrons (leptonic CRs) also produce $\gamma$-rays through two radiative processes: inverse Compton scattering and bremsstrahlung.

The emissivity of inverse Compton scattering depends on the density of relativistic electrons and the number density and spectrum of the background photons that are being upscattered. The relation between initial photon energy and up-scattered energy is $h \nu_{\mathrm{IC}} \approx \gamma_{\mathrm{e}}^{2} h \nu_{i}$. The background radiation is often assumed to be from the cosmic microwave background, which usually dominates the photon field in the Galaxy. But in some cases other sources of photons dominate. A case in point is Cas A, which is itself a strong source of far infrared radiation, which is the dominant source of seed photons for inverse Compton $\gamma$-rays in the object (e.g. Abdo et al. 2010b).

The cross section of inverse Compton scattering is given by the Thomson cross section $\sigma_{\mathrm{T}}$, except at very high energies where the cross is reduced as a result of the Klein-Nishina effect. Thus the total electron energy loss due to inverse Compton scattering is approximately

$\frac{d E_{\mathrm{e}}}{d t}=-\frac{4 \pi}{c} \sigma_{\mathrm{T}} u_{\mathrm{rad}} \gamma_{\mathrm{e}}^{2}$

with $u_{\mathrm{rad}}$ the radiation density of background photons. Since $\nu_{\mathrm{IC}} \propto E_{\mathrm{e}}^{2}$ (so $\left.d E \propto \nu^{-1 / 2} d \nu\right)$, the relation between the photon index and the electron spectral index is $\Gamma=\left(q_{\mathrm{e}}+1\right) / 2$, with $q_{\mathrm{e}}$ the spectral index of the electrons. This

7 Sometimes the effect of multiplicity and cross section is combined in an inclusive cross section $\sigma\left(E_{\mathrm{p}, \text { incl }}\right)$ (e.g. Dermer, 1986, Kamae et al., 2006). 


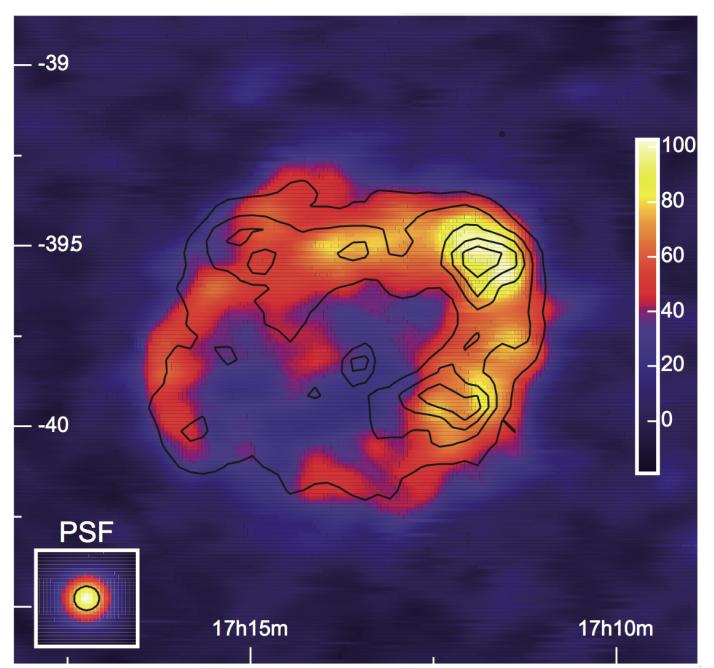

Fig. $12 \gamma$-ray excess count map of RX J1713.7-3946 as observed by HESS. Overlayed are 1-3 keV X-ray ( $A S C A$ ) intensity contours. Credit: Aharonian et al., A\&A, 235, 243, 2007, reproduced with permission (C) ESO.

relation between the spectral indices of the photon and electron spectrum is the same as for synchrotron radiation (Ginzburg and Syrovatskii, 1965), and is quite distinct from the relation between primary spectrum and photon spectrum expected from pion decay. Note that over a large range the electron and proton spectral indices are expected to be similar with $q_{\mathrm{e}}=q_{\mathrm{p}} \approx 2$. The inverse Compton spectrum is expected to be harder $\Gamma \approx 1.5-1.7$, but in the $\mathrm{TeV}$ range of the spectrum the cut-off of the electron spectrum can still result in a spectrum softer than $\Gamma=1.7$. For that reason, the broad coverage provided by including $\mathrm{GeV}$ observations with Fermi or AGILE is important. Moreover, the GeV emission of supernova remnants comes from the same electrons that also produce radio synchrotron radiation. So the spectral slopes in the radio and $\gamma$-rays can be directly compared.

A third radiation process that is important, and which like pion-decay scales with background density $n_{\mathrm{H}}$, is bremsstrahlung, caused by deflections of the electrons as they encounter charged particles. For relativistic electrons the power-law spectral photon index is approximately equal to the primary electron spectrum, $\Gamma=q_{\mathrm{e}}$. The bremsstrahlung is approximately a power law over a broad range of energies, unlike pion decay, which drops off rapidly for energies below half the pion rest mass energy of neutral pions $65.5 \mathrm{MeV}$.

\subsection{2 $\gamma$-ray observation of young shell-type supernova remnants}

The first shell-type supernova remnant to be detected in ultra high energy $\gamma$-rays was Cas A with the HEGRA single-dish Cherenkov telescope (Aharonian et al. (2001). With the advent of more sensitive telescopes like HESS and later $M A G I C$ and VERITAS, more shell-type supernova remnants were detected (Table 1.1). These include well known sources such as Cas A (Aha- 
ronian et al., 2001; Albert et al. 2007b), SN 1006 (Acero et al., 2010), RCW 86 (Aharonian et al., 2009) and very recently Tycho (Acciari et al., 2011). In addition, there are a number of previously poorly known supernova remnants detected in TeV $\gamma$-rays. These include RX J1713.7-3946 (Aharonian et al. 2004) and RX J0852.0-4622 (HESS Collaboration: F. A. Aharonian, 2006). These two sources have a low surface brightness in the radio and $\mathrm{X}$ rays, but have turned out to be among the brightest $\mathrm{TeV}$ sources in the Galaxy. All these TeV sources are also known as X-ray synchrotron emitters, with the X-ray emission from RX J1713.7-3946 and RX J0852.0-4622 being even completely dominated by synchrotron emission (Sect. 2.3). The average power-law index of the $\gamma$-ray emission from young shell-type supernova remnants is $\Gamma \approx 2.3 \pm 0.3$. The hardest emission was detected for $\mathrm{RX}$ J1713.7-3946, with emission detected up to $\sim 100 \mathrm{TeV}$. Its average spectrum is best fit with $\Gamma=2.32 \pm 0.01$. But a better fit is provided by a power law with an exponential cut-off that scales as $\exp \left(-\sqrt{\nu / \nu_{\mathrm{c}}}\right)$. In that case the best fit parameters are $\Gamma=1.79 \pm 0.06$, a cut-off energy of $h \nu_{\mathrm{c}}=3.7 \pm 1.0 \mathrm{TeV}$.

Initially a strong case was made that the $\mathrm{TeV} \gamma$-ray emission was caused by pion-decay radiation (e.g. Berezhko and Völk, 2008, 2010). This would constitute direct proof that protons are accelerated to energies of at least $\sim 10^{14} \mathrm{eV}$. As discussed in Sect. 2.3 this implied that the magnetic field must be relatively high in all these sources $(\gtrsim 100 \mu \mathrm{G})$, as otherwise the observed synchrotron flux would require a high $\mathrm{CR}$ electron density. This would enhance the $\gamma$-ray inverse Compton scattering, and making it the dominant source of $\gamma$-rays.

The fact that all young supernova remnants that emit $\mathrm{TeV} \gamma$-rays are also X-ray synchrotron emitters (Table 1.1) is a reason to be cautious about pion-decay dominated models, since, clearly, electrons are present with energies $>\mathrm{TeV}$, which could result in an appreciable, if not dominant, inverse Compton contribution to the $\gamma$-ray flux. Moreover, the lack of thermal X-ray emission from the prominent $\gamma$-ray source RX J1713.7-3946 suggests that the density in the supernova remnant is low. This would suppress pion-decay emission, as it scales with $n_{\mathrm{H}}$ (Eq. 37, see Katz and Waxman, 2008, Ellison et al. 2010). But it may be that the thermal X-ray emission is suppressed by very low plasma temperatures, which could be a by-product of efficient, non-linear CR acceleration (Drury et al. 2009). As explained in Sect. 2.3 the magnetic field in RX J1713.7-3946 may not be as high as sometimes assumed. This makes it also more likely that inverse Compton scattering dominates the TeV emission from this source.

The best evidence that the $\mathrm{TeV} \gamma$-ray emission from RX J1713.7-3946 is dominated by inverse Compton scattering is provided by recent Fermi observations (Abdo and Fermi LAT Collaboration, 2011), because they provided the broad spectral coverage needed to distinguish the different spectral behavior of pion-decay and inverse Compton scattering. This showed that $\Gamma=1.5$, which is consistent with the spectral index expected for inverse Compton scattering. The hadronic-model (pion-decay) for the $\gamma$-ray is not yet completely ruled out, as $\Gamma=1.5$ is still consistent with a very hard primary proton spectrum of $q_{\mathrm{p}}=1.5$, which could be the result of extremely efficient, non-linear CR acceleration (Malkov, 1997). But in that case the 
spectral break around $4 \mathrm{TeV}$ indicates that the maximum proton energy is around $40 \mathrm{TeV}$, very far removed from "the knee". So ironically, a leptonic model may be more consistent with the idea that supernova remnants accelerate protons to very high energies, as it gives the freedom to assume that the non-detected protons have much higher cut-off energies.

Cas A was the first shell-type supernova remnant detected in $\mathrm{TeV} \gamma$-rays (Aharonian et al., 2001, Albert et al., 2007b). Its broad band $\mathrm{GeV} / \mathrm{TeV} \gamma$-ray spectrum casts some doubt on the idea that more than $10 \%$ of the explosion energy in young supernova remnants is contained by CRs Abdo et al. 2010b). The recent Fermi observations do not provide sufficient information on the dominant $\gamma$-ray emission, as both hadronic (pion decay) models and leptonic models (for Cas A a combination of inverse Compton scattering and bremsstrahlung) provide reasonable fits to the $0.1 \mathrm{GeV}-10 \mathrm{TeV}$ spectrum. But for Cas A the conclusion is that either model shows that the CR energy budget in Cas $\mathrm{A}$ is at most $4 \times 10^{49} \mathrm{erg}$, which corresponds to at best $2 \%$ of the total explosion energy.

Of all the young supernova remnants, only Tycho's supernova remnant seems to offer a good case that a substantial amount of the explosion energy has been used to accelerate CRs, and that the $\gamma$-ray emission is dominated by pion-decay. Tycho's supernova remnant was only recently detected in the TeV and GeV range by respectively VERITAS (Acciari et al., 2011) and Fermi (Giordano et al., 2011). The spectrum in the GeV range has a spectral index of $\Gamma=2.2$, whereas the radio spectral index $\alpha=0.6$ implies an inverse Compton scattering spectrum of $\Gamma_{\mathrm{IC}}=1.6$. Non-thermal bremsstrahlung could in principle fit the spectrum, but requires a density of $n_{\mathrm{H}} \approx 9 \mathrm{~cm}^{-3}$. This would be much higher than expected based on the dynamics of the supernova remnant (Katsuda et al., 2010a), unless the compression ratio behind the shock front is very high. The total energy in CRs required by fitting a pion-decay model to the joint Fermi and VERITAS data shows that $10 \pm 5 \%$ of the explosion energy is contained in CRs. The uncertainty is caused by some uncertainties in the distance, density, and overall explosion energy.

So what are we to make of the $\gamma$-ray emission from young shell-type supernova remnants? Taking all the $\gamma$-ray observations of young supernova remnants together, the results are somewhat mixed and confusing. Sources that initially were considered promising candidates for substantial hadronic CR acceleration, RX J1713.7-3946 and Cas A, do not seem to require a substantial energy in CRs. But Tycho's supernova remnant seems to have a substantial energy in CRs, although perhaps not as high as one might hope for in a young supernova remnants. Currently, only a few $\mathrm{TeV}$ emitting supernova remnants have also been detected by Fermi, so the balance may still shift a little more toward a hadronic origin for $\gamma$-ray emission.

However, an important ingredient of CR acceleration by supernova remnants has only recently received more attention. This is the issue of CR escape. Non-linear CR acceleration is thought to be accompanied by substantial escape of high energy particles upstream (e.g. Malkov and Drury, 2001; Blasi et al., 2005, Reville et al., 2009, Vink et al. | 2010; Drury, 2011, the latter for a more nuanced view of escape). It is actually this escape of CRs 
that makes the high compression ratios possible. It is therefore possible that for sources like Cas A a substantial amount of CR energy has already escaped the supernova remnant. Also the discovery of $\mathrm{TeV}$ sources in the vicinity of mature supernova remnants testifies of the importance of CR escape (see the next section).

Note that core-collapse supernova remnants such as Cas A and Type Ia supernova remnants, such as Tycho, show a different $\gamma$-ray response to escaping CRs. The reason is that core-collapse supernova remnants often evolve in the wind of their progenitors, which in the case of Cas A is likely a dense red supergiant wind (Chevalier and Oishi, 2003). In such a wind the density falls off with radius as $\rho(R)=\dot{M}_{\mathrm{w}} /\left(4 \pi R^{2} v_{\mathrm{w}} / 3\right)$, or, $n_{\mathrm{H}}(R) \propto R^{-2}$. So escaping CRs, while diffusing outward, encounter less dense material. If we assume that the total number of CRs is $N_{\mathrm{cr}}$ and the typical region over which they have diffused is $R_{\text {diff }}$ than the average CR density is $n_{\mathrm{cr}}=$ $3 N_{\mathrm{cr}} /\left(4 \pi R_{\mathrm{diff}}^{3}\right)$. The pion decay luminosity scales approximately as

$L_{\pi^{0}} \propto \int_{0}^{R_{\mathrm{diff}}} n_{\mathrm{cr}} n_{\mathrm{H}}(R) 4 \pi R^{2} d R$.

For a uniform background density this means that

$L_{\pi^{0}} \propto N_{\mathrm{cr}} n_{\mathrm{H}}$,

i.e. the total luminosity does not depend on $R_{\text {diff }}$. But for a supernova remnant evolving inside a stellar wind we have

$L_{\pi^{0}} \propto 3 N_{\mathrm{cr}} \dot{M}_{\mathrm{w}} /\left(v_{\mathrm{w}} R_{\mathrm{diff}}^{2}\right)$.

Since $R_{\text {diff }} \propto \sqrt{t}$, this means that for core-collapse supernova remnants, the older the supernova remnant, the weaker the pion decay luminosity. This simple model does, of course, assume that the highest energy CRs are accelerated rather early in the life of a supernova remnant. For a supernova remnant inside a stellar wind that may well be the case, because the density and velocity are very high early on, and all the flux of particles entering the shock is higher in the beginning (see the case of SN 1993J in Sect. 2.3). This is in contrast to the case of a uniform medium, where the flux of particles entering the shock is slowly increasing with time ${ }^{8}$ This difference in acceleration properties for supernova remnants in different media will likely lead to larger maximum proton energies in core collapse supernova remnants, especially those expanding in dense winds, and Type Ia supernova remnants (Ptuskin and Zirakashvili, 2005, Schure et al., 2010).

If escape is indeed important, even for young supernova remnants, this implies that perhaps for Tycho's supernova remnant some of the $\gamma$-ray emission may not come from the shell, but from the vicinity of the supernova remnant. Indeed, there is a hint of an offset between Tycho's supernova remnant and

8 For example, for the Sedov solution in a uniform medium, $R \propto t^{2 / 5}$ we have $F=4 \pi n_{\mathrm{H}}(R) R^{2} v \propto t^{1 / 5}$. Whereas for the Sedov solution inside a stellar wind, $R \propto t^{2 / 3}$, we get $F=4 \pi n_{\mathrm{H}}(R) R^{2} v \propto v \propto t^{-1 / 3}$. 
the $\mathrm{TeV} \gamma$-ray emission in the direction of a molecular cloud Acciari et al. 2011).

The above analysis also implies that supernova remnants in a uniform density medium should still be visible for a relatively long time, as the total pion-decay luminosity is more or less constant. But in practice, at some moment the total emission may come from such a large region that the $\gamma$-ray surface brightness will become low, and the supernova remnant CR "halo" will be difficult to detect, due to lack of contrast with the background emission. Moreover, at large distance from the supernova remnant the diffusion coefficient is likely to become larger, as the CR generated, magnetic turbulence will be reduced if the CRs are spread out. Furthermore, the circumstellar media of supernovae are usually more complicated than the simple models presented here. In particular, a stellar wind zone finishes in a region of enhanced density, the wind shell. This shell, once reached by escaping CRs. may be a source of enhanced pion decay emission.

Over the next five years we will likely increase our understanding of $\gamma$-ray emission of young supernova remnants, as the current $\gamma$-ray observatories will continue their exploration of the Galaxy. But then the next big step forward will likely be made with the Cherenkov Telescope Array (CTA: CTA Consortium, 2010), which will be ten times more sensitive, and have a broader energy range than current Cherenkov telescopes. This may provide more information on the importance of escaping CRs, and how they diffuse into and interact with the circumstellar medium, before becoming part of the overall population of Galactic CRs.

\subsection{GeV and TeV $\gamma$-ray observations of mature SNRs}

The first evidence that old supernova remnants can be GeV emitters too, was provided by Esposito et al. (1996), based on EGRET data. However, the point spread function of EGRET did not allow for an unambiguous identification of the $\gamma$-ray source with supernova remnants. Currently, Fermi has been able to confirm the EGRET results and has added many other mature supernova remnants to the list of the $\gamma$-ray sources (Table 2.14).

Their $\gamma$-ray spectra are generally characterized by a broken power law with the peak of the emission in the GeV regime (e.g. Li and Chen, 2011). The break photon energy is typically a few $\mathrm{GeV}$ and spectra above the break are very steep in comparison to the spectra as predicted by diffusive shock acceleration theory. For this reason, the GeV $\gamma$-ray telescopes as Fermi and $A G I L E$ are essential for detecting these intermediate aged remnants. Remarkably, shock waves of almost all these old remnants are interacting with dense molecular clouds, as evidenced by detection of hydroxyl $(\mathrm{OH})$ masers at $1720 \mathrm{MHz}$ (Wardle and Yusef-Zadeh, 2002, Hewitt et al., 2009). The origin of the $\mathrm{GeV} \gamma$-rays can be interpreted as hadronic processes (e.g. Abdo et al. 2010a). The high density in and near the supernova remnant suggests that the emission is likely due to pion decay, but bremsstrahlung cannot in all cases be ruled out, as this also scales with $n_{\mathrm{H}}$ (e.g., Abdo et al., 2010d, and section 2.13. However, new AGILE observations of two supernova remnants 


\begin{tabular}{|c|c|c|c|c|}
\hline Name & $\begin{array}{l}\text { Other } \\
\text { name }\end{array}$ & $\begin{array}{l}\mathrm{GeV} \\
\gamma \text {-rays }\end{array}$ & $\begin{array}{l}\mathrm{TeV} \\
\gamma \text {-rays }\end{array}$ & Interacting? \\
\hline G6.4-0.1 & W28 & $\mathrm{Y}^{1}$ & $\mathrm{Y}^{2}$ & $\mathrm{OH}$ maser $^{3}$ \\
\hline G8.7-0.1 & W30 & $\mathrm{Y}^{4}$ & $\mathrm{~N}$ & $\mathrm{OH}$ maser $^{3}$ \\
\hline G15.4+0.1 & & $\mathrm{N}$ & $Y^{5}$ & \\
\hline G22.7-0.2 & & $\mathrm{Y}^{6}$ & $\mathrm{~N}$ & $\mathrm{~N}$ \\
\hline G23.3-0.3 & W41 & $Y^{7}$ & $\mathrm{Y}^{9}$ & $\mathrm{CO}^{8}$ \\
\hline $\mathrm{G} 31.9+0.0$ & 3C 391 & $\mathrm{Y}^{4}$ & $\mathrm{~N}$ & OH-maser ${ }^{3}$ \\
\hline G34.7-0.4 & W44 & $\mathrm{Y}^{9}$ & $\mathrm{~N}$ & $\mathrm{OH}-\mathrm{maser}^{10}$ \\
\hline G43.3-0.2 & W49B & $\mathrm{Y}^{11}$ & $\mathrm{Y}^{12}$ & $\mathrm{H}_{2}$ emission ${ }^{13}$ \\
\hline G49.2-0.7 & W51C & $\mathrm{Y}^{14}$ & $\mathrm{Y}^{15}$ & OH-maser ${ }^{3}$ \\
\hline G74.0-8.5 & Cygnus Loop & $\mathrm{Y}^{15}$ & $\mathrm{~N}$ & $\mathrm{CO}$ emission $^{16}$ \\
\hline G180.0-1.7 & S147 & $\mathrm{Y}^{17}$ & $\mathrm{~N}$ & \\
\hline G189.1+3.0 & IC 443 & $\mathrm{Y}^{18}$ & $\mathrm{Y}^{19}$ & $\mathrm{OH}-$ maser $^{20}$ \\
\hline G304.6+0.1 & Kes 17 & $\mathrm{Y}^{21}$ & $\mathrm{~N}$ & $\mathrm{OH}-$ maser $^{22}$ \\
\hline G318.2+0.1 & & $\mathrm{N}$ & maybe 23 & ${ }^{12} \mathrm{CO}$ emission $^{23}$ \\
\hline G348.5+0.1 & CTB 37A & $\mathrm{Y}^{4}$ & $\mathrm{Y}^{24}$ & $\mathrm{OH}-$ maser $^{22}$ \\
\hline G349.7+0.2 & & $\mathrm{Y}^{4}$ & $\mathrm{~N}$ & ${ }^{12} \mathrm{CO}$ emission $^{25}$ \\
\hline G359.1-0.5 & HESS J1745-303 & $\mathrm{N}$ & $\mathrm{Y}^{26}$ & $\mathrm{OH}-\operatorname{maser}^{27}$ \\
\hline
\end{tabular}

Table 4 Table with mature supernova remnants that have been detected at $\mathrm{GeV}$ and/or TeV $\gamma$ energies. ${ }^{1}$ Abdo et al. (2010a); Giuliani et al. (2010), ${ }^{2}$ Aharonian et al. (2008c), ${ }^{3}$ Hewitt et al. (2009), ${ }^{4}$ Castro and Slane (2010), ${ }^{5}$ Hofverberg et al. (2011b) ${ }^{6}$ Laffon et al. (2011), ${ }^{7}$ Méhault et al. (2010), ${ }^{8}$ Tian et al. (2007), ${ }^{9}$ Abdo et al. (2010d); Giuliani et al. (2011), ${ }^{10}$ Hoffman et al. (2005), ${ }^{11}$ Abdo et al. (2010c), ${ }^{12}$ Brun et al. (2011), ${ }^{13}$ Keohane et al. (2007), ${ }^{14}$ Abdo et al. (2009), ${ }^{15}$ Fiasson et al. (2008), ${ }^{16}$ Katagiri et al. (2011), ${ }^{17}$ Katsuta et al. (2012), ${ }^{18}$ Abdo et al. (2010e); Tavani et al. (2010), ${ }^{19}$ Albert et al. (2007a); Acciari et al. $(2009)^{20}$ Hewitt et al. (2006), ${ }^{21} \mathrm{Wu}$ et al. (2011), ${ }^{22}$ Frail et al. (1996), ${ }^{23}$ Hofverberg et al. (2011a), ${ }^{24}$ Aharonian et al. (2008a), ${ }^{25}$ Dubner et al. (2004), ${ }^{26}$ Aharonian et al. (2008b), ${ }^{27}$ Wardle and Yusef-Zadeh (2002). For a short descripion of most of these sources, see L1 and Chen (2011).

(W44, W28 Giuliani et al., 2011, 2010) reveal the characteristic low energy cut-off expected for pion decay (Figure 13).

Several models explain the break and the steep spectra in terms of a crushed cloud model (Aharonian and Atoyan, 1996; Ohira et al., 2011a; Malkov et al., 2011: Uchiyama et al., 2010; Inoue et al. 2010). In this model, the $\mathrm{GeV}$ and $\mathrm{TeV} \gamma$-ray emission is explained by pion decay from accelerated protons after the supernova remnant interacts with a high density molecular cloud.

As CRs escape from remnants, they have a spectrum with a power-law index of approximately two (Ptuskin and Zirakashvili, 2005). Aharonian and Atoyan (1996) showed that $\gamma$-ray spectra CRs after escaping the remnant get steeper because of energy-dependent diffusion. The diffusion length after escaping from the supernova remnant, $R_{\text {diff }}$, is given by

$R_{\mathrm{diff}}(E)=\sqrt{4 D_{\mathrm{ISM}}(E) \Delta t}$,

where $D_{\mathrm{ISM}}(E)$ and $\Delta t$ are the diffusion coefficient of ISM and the elapsed time, respectively. Let $Q_{\mathrm{SNR}} \propto E^{-s}$ and $D_{\mathrm{ISM}}(E) \propto E^{\delta}$ be the CR spectrum 


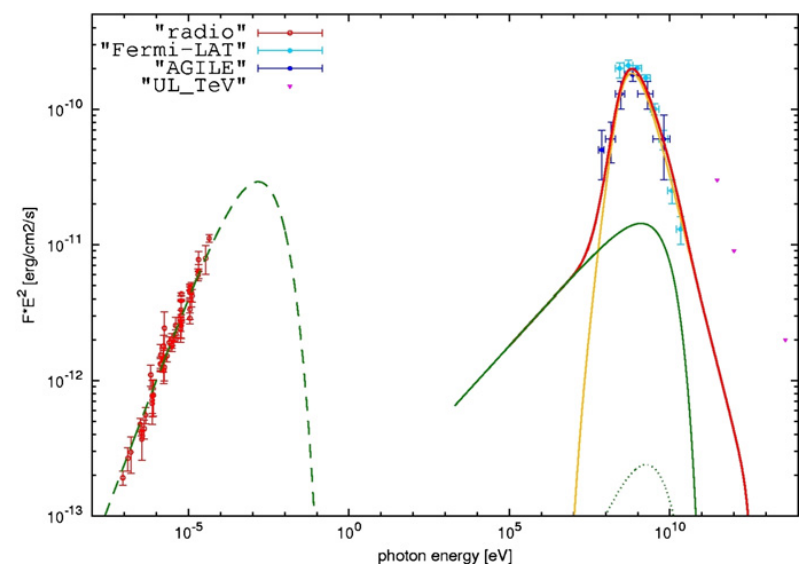

Fig. 13 Broadband spectrum of supernova remnant W44 with the best-fit model (red line). Overplotted is a hadronic model, revealing the characteristic low cut-off expected for pion decay (yellow curve). The green curves show the contribution of several emission mechanisms from energetic electrons (Figure from Giuliani et al. 2011, reproduced by permission of the AAS.)

in the supernova remnant and the diffusion coefficient of ISM, respectively. Then, the CR spectrum at a certain point within the diffusion length, $f_{\text {diff }}$, is given by

$f_{\text {diff }}(E) \propto \frac{Q_{\mathrm{SNR}}(E)}{R_{\mathrm{diff}}^{3}(E)} \propto E^{-(s+1.5 \delta)}$.

The factor of $R_{\text {diff }}^{-3}$ can be interpreted as the dilution due to the diffusion. Higher energy CRs have a longer diffusion lengths and are more diluted, so that the spectrum of escaping CRs becomes steeper than that of the source. The interaction between these escaping CRs and molecular clouds can produce $\gamma$-rays outside supernova remnants and can explain the unidentified very-high-energy $\gamma$-ray sources (Aharonian and Atoyan, 1996, Gabici et al. 2009). Interestingly, $H E S S$ and $M A G I C$ have now revealed several TeV sources, near, but not coinciding with, mature supernova remnants. This appears to be the case for W28 (Aharonian et al., 2008c), IC 443 (Albert et al., 2007a) and G35.6-0.4 (Torres et al., 2011). The idea is that the sources are molecular clouds that are illuminated as CRs that escaped from the supernova remnant are interacting with the dense material in the molecular cloud. Escaping CR electrons also produce $\gamma$-rays outside supernova remnants (Ohira et al. 2011b).

In summary, it is unclear whether the $\mathrm{GeV}$ and $\mathrm{TeV} \gamma$-ray emission from the youngest remnants is of hadronic of leptonic origin. In contrast, the GeV (and $\mathrm{TeV}$ ) $\gamma$-ray emission from middle-aged supernova remnants is most likely explained by the hadronic scenario, giving credence to the paradigm that supernova remnants are capable of accelerating protons as well as electrons. As these old remnants are expected to no longer accelerate particles up to the knee, this does not provide evidence for supernova remnants being 
able to accelerate particles to the knee. But since the new $\mathrm{GeV} / \mathrm{TeV}$ observations provide clear evidence for escaping CRs, acceleration up to or even beyond the knee is certainly possible.

\section{Balmer dominated Shocks}

A fast non-radiative shock in ionized gas produces none of the usual nebular emission lines, but if the gas is partly neutral, then some $\mathrm{H}$ atoms will be excited before they are ionized. They produce faint Balmer line emission filaments that trace the edges of several young Type I supernova remnants, along with a few older remnants and pulsar wind bow shocks. The $\mathrm{H} \alpha$ line profiles show broad and narrow components (Kirshner and Chevalier, 1978), which provide valuable diagnostics for the proton and electron temperatures in the shocked gas, along with a means of detecting a shock precursor (Raymond, 1991; Heng, 2010).

The neutrals pass through the shock front completely unaffected by the magnetic and electric fields in the shock or by plasma turbulence. They find themselves immersed in hot plasma moving at $V_{s}(1-1 / r)$, where $V_{s}$ is the shock speed and $r$ is the compression ratio. One of three things can happen: they can be ionized by collisions with electrons or ions; they can be excited by electrons or ions, or they can undergo charge transfer with protons. Eventually they all become ionized. All the H I emission is produced before the hydrogen is ionized, so it reflects conditions immediately behind the shock.

Particles that are excited before any other interaction occurs produce Balmer line emission with the same velocity distribution that they had upstream, and this is called the Narrow Component of the profile. Since the upstream plasma must be cooler than about $10,000 \mathrm{~K}$ in order to have a substantial neutral fraction, one expects a line width below $25 \mathrm{~km} \mathrm{~s}^{-1}$ (FWHM). Other particles undergo charge transfer. This produces a population of neutrals with a velocity distribution similar to that of the post-shock protons, but weighted by the velocity times the charge transfer cross section. In shocks faster than about $1000 \mathrm{~km} \mathrm{~s}^{-1}$, the charge transfer can go into an excited state and produce an $\mathrm{H} \alpha$ photon. Either charge transfer to the excited states or collisional excitation of the fast neutrals will produce a Broad Component, whose line width is on the order of the post-shock proton thermal speed (Chevalier and Raymond, 1978; Chevalier et al., 1980, Heng and McCray, 2007; Heng et al., 2007; van Adelsberg et al., 2008).

The line widths of the Narrow and Broad components give the pre- and post-shock temperatures fairly directly. The intensity ratio of the Broad and Narrow components depends on the ratio of ionization and charge transfer rates, so it provides a means of determining the electron-to-ion temperature ratio immediately behind the shock. In the following, we discuss the implications for shock precursors and for electron-ion temperature equilibration. 


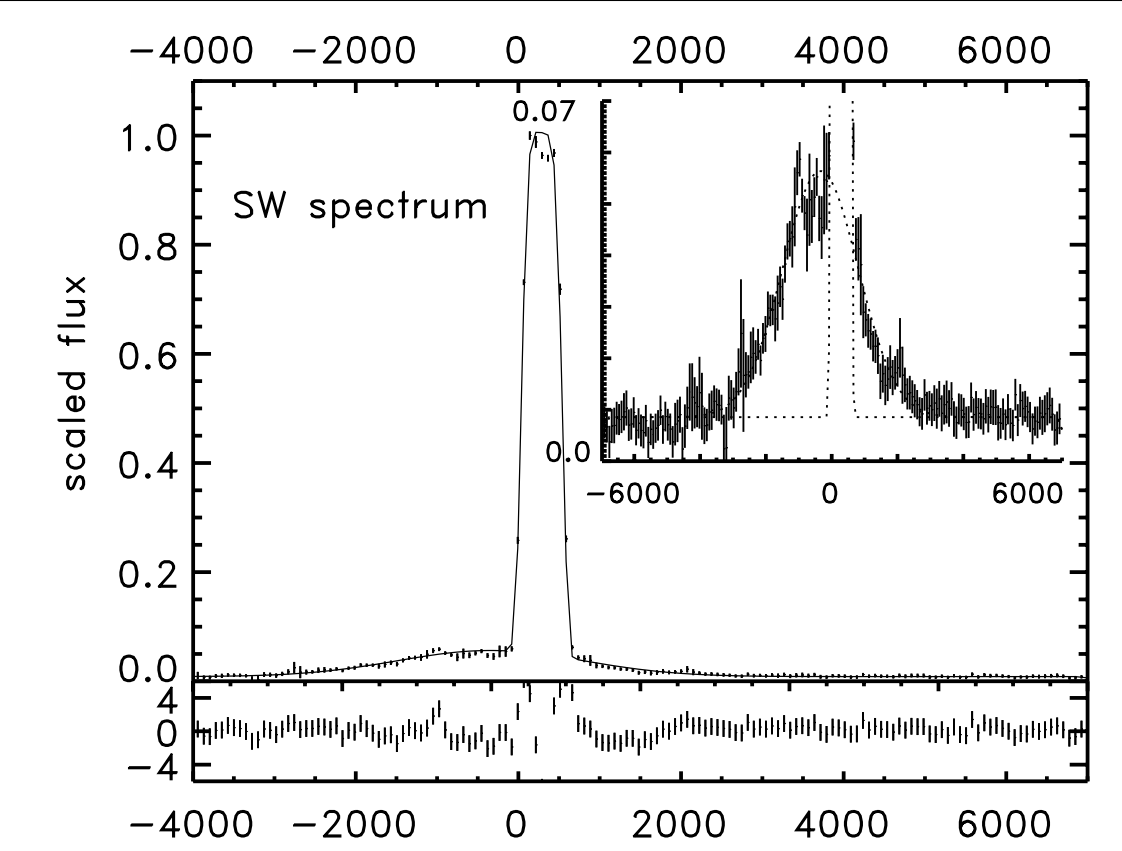

Fig. $14 \mathrm{H} \alpha$ line emission of the southwest shock of supernova remnant 0509-67.5 in the Large Magellanic Cloud. The solid line indicates a fit to the line, with a broad and a narrow gaussian line profile convolved with the spectral resolution. Note that the width of the narrow component is determined by the spectral resolution. Figure was published in Helder et al. (2010), reproduced by permission of the AAS.

\subsection{Anisotropy and non-Maxwellian distributions}

Nearly all analyses of X-ray, optical and UV emission from shocks assume isotropic, Maxwellian velocity distributions, but in a collisionless plasma that may not be the case. Several types of complex distributions are possible.

Neutrals following charge transfer: Heng and McCray (2007), Heng et al. (2007), van Adelsberg et al. (2008) and de Laat (2011) have carried out detailed calculations of the $\mathrm{H}$ I velocity distribution in the shocked plasma. For moderate shock speeds, the distribution is nearly Maxwellian, but at speeds above about $2000 \mathrm{~km} \mathrm{~s}^{-1}$, the rapid dropoff of the charge transfer cross section with speed produces distributions that are narrower in the flow direction than the perpendicular direction, and they are not Maxwellian.

Pickup Ions: Ohira et al. (2009b) and Raymond et al. (2008) discuss the velocity distribution of protons produced when $\mathrm{H}$ atoms that pass through the shock as neutrals become ionized downstream. Because they suddenly find themselves immersed in a rapidily moving magnetized plasma, they behave like pickup ions in the solar wind (Moebius et al., 1985: Gloeckler et al. 1993; Isenberg, 1995). They initially have the form of a monoenergetic ring beam gyrating around the magnetic field. That unstable distribution generates plasma waves and relaxes to a bispherical distribution symmetric about the magnetic field direction. Ohira et al. (2009b) discuss the wave genera- 
tion, magnetic field amplification and shock structure modification that can result, and those changes could affect $\mathrm{CR}$ acceleration. Raymond et al. (2008) predict the signatures that could be detectable in the $\mathrm{H} \alpha$ line profile.

Power-law tails: cosmic-ray acceleration implies a power-law tail at some level, and velocity distributions in the solar wind are often seen to have a $\kappa$-distribution character, consisting of a nearly Maxwellian core with a powerlaw tail. The power-law tail must contain a large fraction of the total energy in order to produce detectable wings on the $\mathrm{H} \alpha$ profile.

Observations of $\mathrm{H} \alpha$ line profiles are generally interpreted by fitting Gaussians for the Broad and Narrow components, but Raymond et al. (2010) show the profile from a shock in Tycho's supernova remnant that cannot be fit with two Gaussians. They are not able to discriminate among the possibilities that the profile results from pickup ions, a $\kappa$ distribution, a hot precursor, or merely the superposition of different shocks within the spectrograph slit. Analysis of other spectra from Tycho may help to sort out the options.

\subsection{Shock precursors}

The diffusive shock acceleration model for acceleration of CRs in shock waves requires a precursor region where energetic particles streaming away from the shock generate turbulence, and where the turbulence scatters particles back toward the shock so that first order Fermi acceleration can occur (Blandford and Eichler, 1987). The plasma in the precursor is compressed, heated and accelerated toward the shock speed (Boulares and Cox, 1988, Vladimirov et al. 2008). The scale length of the precursor is given by the diffusion coefficient of the most energetic cosmic rays, $D$ (see equation 12), divided by $V_{s}$, and it should be on the order of an arcsecond for typical young Galactic supernova remnants. A precursor unrelated to CRs is also possible, in that a few percent of the neutrals in the Broad Component can overtake the shock, and if they deposit energy and momentum there they can decelerate (in the shock frame) and heat the incoming gas (Hester et al. 1994, Smith et al. 1991). Its length scale is the charge transfer length scale, which is typically 0.1 to 1 arcsecond.

Indirect evidence for a shock precursor comes from the narrow component widths measured in 9 supernova remnants (Sollerman et al., 2003). With the exception of SN1006, the widths are 35 to $58 \mathrm{~km} \mathrm{~s}^{-1}$. At the temperatures implied by those widths, hydrogen would be fully ionized, so the heating must occur in a precursor narrow enough that much of the neutral gas passes through the precursor before being ionized. This implies thicknesses compatible with the $\mathrm{CR}$ precursor, which in turn would imply $D$ values of $10^{24}-10^{25} \mathrm{~cm}^{2} / \mathrm{s}$ (Hester et al., 1994, Smith et al. 1991, Lee et al., 2007). The one supernova remnant that shows no enhanced line width is SN 1006 (Sollerman et al., 2003). This could be related to the lack of non-thermal emission in that section of the supernova remnant, but it might also be that the density is so low that neutrals pass through the precursor without interacting at all, so that their line width does not reflect heating in the precursor. 
More direct evidence for a precursor comes from the observation of faint emission ahead of the main shock filaments in Tycho's supernova remnant. Spectra from Subaru show this to be narrow component emission (Lee et al. 2007), and an HST image shows that it trails off gradually ahead of the shock (Lee et al., 2010). By combining the observations with models by Wagner et al. (2009), Lee et al. (2010) obtained a precursor length scale of about $6 \times 10^{16} \mathrm{~cm}$ and a peak temperature in the precursor of 80,000-100,000 K. This implies $D \sim 10^{25} \mathrm{~cm}^{2} \mathrm{~s}^{-1}$. The neutrals may affect $D$, since they could damp the waves that scatter energetic particles (Drury et al., 1996), which lowers the maximum energy the CRs can achieve and reduces the overall acceleration efficiency.

The interaction between neutrals and a CR precursor may be much more complex than is suggested by the estimates of scale length and temperature. A strong precursor accelerates the pre-shock plasma to a substantial fraction of the post-shock speed. When a neutral becomes ionized in the precursor, either through charge transfer or collisional ionization, it behaves much like a pickup ion in the solar wind (Ohira and Takahara, 2010, Raymond et al., 2011). The resulting population of protons may retain an anisotropic, high speed velocity distribution, or it may come into equilibrium with the thermal protons by way of plasma wave interactions. In either case, if the neutral fraction is substantial there can be important effects on the heating and compression in the precursor and on the injection of particles into the acceleration process.

It should be kept in mind that Broad component neutrals that overtake the shock, as suggested by Hester et al. (1994) and Smith et al. (1991) might also account for the observed Narrow component width of $\mathrm{H} \alpha$ and for the $\mathrm{H} \alpha$ ahead of the main shock front observed by Lee et al. (2007, 2010). Complete models are not yet available, though Lim and Raga (1996) found that this process had little effect on the $\mathrm{H} \alpha$ profile. However, they did not consider the possibility that the protons formed from fast neutrals that penetrate the shock deposit a significant fraction of their energy by generating plasma waves.

\subsection{Temperature versus shock speed}

Once a shock deposits a substantial fraction of its kinetic energy into accelerating particles, the structure of the shock will change. A particle accelerating shock will have a lower post-shock temperature that a non-accelerating shock. In this section, we first describe how the post-shock temperature relates to the post-shock cosmic-ray pressure and cosmic-ray energy flux escaping the system 3.3.1). In 3.3.2 we describe how the proton and electron temperatures can differ and where this causes caveats in determining the cosmic-ray acceleration efficiency. Section 3.3.3 lists the observations that have been carried out to determine cosmic-ray acceleration efficiency parameters based on post-shock plasma temperatures. Section 3.3.4 concludes with the relation between the post-shock pressure and the cosmic-ray energy flux escaping the system. 


\subsubsection{Physical parameters}

If a shock accelerates CRs, it changes the physical parameters of the plasma behind the shock front. Firstly, the CR pressure increases as the shock is accelerating more efficiently. The total pressure behind the shock front remains approximately constant, implying that the local thermal pressure, and therewith the temperature, drops. Secondly, the effective equation-of-state $\left(\gamma_{\mathrm{s}}\right)$ of the post-shock plasma changes from $\gamma_{\mathrm{s}}=5 / 3$ (non-relativistic) to $\gamma_{\mathrm{s}}=4 / 3$ (relativistic) if the post-shock pressure gets more CR dominated (Chevalier, 1983):

$\gamma_{\mathrm{s}}=\frac{\gamma_{\mathrm{s}}}{\gamma_{\mathrm{s}}-1}=\frac{5+3 w_{\mathrm{CR}}}{3\left(1+w_{\mathrm{CR}}\right)}$

Here, $w_{\mathrm{CR}}$ is the fraction of the post-shock pressure that is contributed by CRs. Using the conservation of mass, momentum and energy over the shock front, and taking into account that a fraction $\left(\epsilon_{\mathrm{CR}}\right)$ of the energy flux through the shock $\left(\frac{1}{2} \rho V_{\mathrm{s}}^{3}\right)$ is escaping the system, we get the following expression for the compression ratio over the shock front (c.f., Berezhko and Ellison, 1999 Vink, 2008a):

$r=\frac{G+\sqrt{G^{2}-\left(1-\epsilon_{\mathrm{CR}}\right)(2 G-1)}}{1-\epsilon_{\mathrm{CR}}}$

where $G=\frac{3}{2} w_{\mathrm{CR}}+\frac{5}{2}$. Altogether, this leads to a mean post-shock temperature $(T)$ of

$\langle k T\rangle=\left(1-w_{\mathrm{CR}}\right) \frac{1}{r}(1-1 / r) \mu m_{\mathrm{p}} V_{\mathrm{s}}^{2}$.

Here, $\mu$ is the mean particle mass in units of the proton mass $\left(m_{\mathrm{p}}\right)$, which is $\sim 0.6$ for solar abundances. This has to be compared to the temperature behind non-accelerating shocks: $\langle k T\rangle=3 / 16 \mu m_{\mathrm{p}} V_{\mathrm{s}}^{2}$. To quantify the effect of $\mathrm{CR}$ acceleration, we define a parameter

$$
\beta=\langle k T\rangle / 3 / 16 \mu m_{\mathrm{p}} V_{\mathrm{s}}^{2} .
$$

So, for a non-accelerating shock, $\beta=1$ and a lower $\beta$ implies more efficient shock accelerating CRs.

\subsubsection{Electron-proton temperature equilibration}

To measure the cosmic-ray acceleration efficiency from the temperature deficit, we have to know the mean plasma temperature. Most young supernova remnants emit thermal X-ray emission, enabling us to determine their electron temperatures from the shape of the bremsstrahlung continuum. Unfortunately, directly behind the shock front, the electron temperature is not necessarily equal to the temperature of other atomic species. They might even 


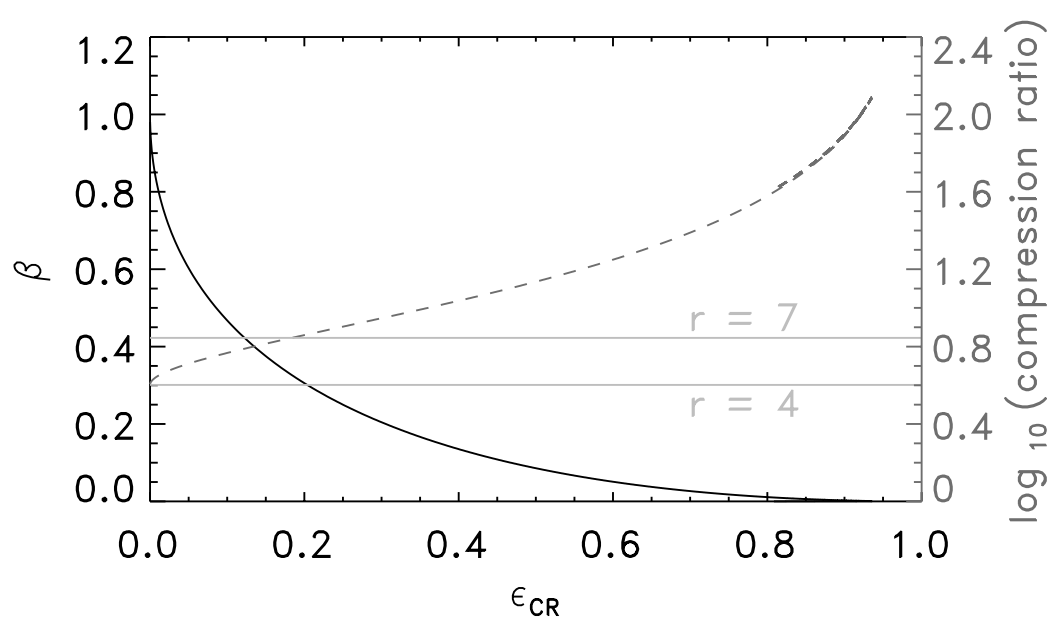

Fig. $15 \beta$ (black line) and compression ratio (grey dashed line) over the shock front as function of energy flux escaping the shock (as fraction of the incoming energy, $F_{\text {TОт }}=\frac{1}{2} \rho V_{\mathrm{s}}^{3}$ ). Based on the equations of Vink et al. (2010).

differ as much as their mass ratio (a factor of 1836 for electrons to protons). Therefore, the electron temperature at the shock front is a poor indicator for the mean plasma temperature.

In time, the temperatures will evolve to the mean plasma temperature. If Coulomb interactions are the only process contribution to equilibration, this will happen after $n_{\mathrm{e}} t \sim 10^{12} \mathrm{~s} \mathrm{~cm}^{-3}$, where $n_{\mathrm{e}}$ is the electron density, and $s$ the time in seconds (Vink, 2004). However, it is rather uncertain at which electron to proton temperature ratio a plasma has if it was just shocked. From optical observations, a relation of $1 / V_{\mathrm{s}}^{2}$ was proposed for $V_{\mathrm{s}}>400 \mathrm{~km} \mathrm{~s}^{-1}$, below $400 \mathrm{~km} \mathrm{~s}^{-1}$ the electron and proton temperature were similar (Ghavamian et al. 2007) (however, see van Adelsberg et al. 2008: Helder et al. 2011). This change of behavior at $400 \mathrm{~km} \mathrm{~s}^{-1}$ might be explained if one realizes that this if very similar to the electron velocities in a neutral medium with a temperature of $10^{4} \mathrm{~K}$. Therefore, the electrons might not feel the shock as a discontinuity (Bykov and Uvarov, 1999, Bykov, 2004).

It is not well-understood how cosmic-ray acceleration affects ion-electron temperature equilibration. One possibility is that the heating of electrons in the CR precursor in combination with the lower Mach number will lead to temperature equilibration for shocks with velocities $>400 \mathrm{~km} \mathrm{~s}^{-1}$. Additionally, Patnaude et al. (2009) argue that the increased compression ratio of particle accelerating shocks (section 4 ) accelerates the equilibration, as the ionization time $\left(n_{\mathrm{e}} t\right)$ is a function of the density too.

\subsubsection{Observations}

Hughes et al. (2000) measured the electron temperature behind the shock front of SNR 0102.2-7210 in the small Magellanic Cloud as well as the shock velocity based on X-ray data. They found that, even after correcting for 
temperature equilibration effects, the measured electron temperature was still too low for the measured shock velocity, indicating that SNR 0102.27210 has efficiently accelerating shocks.

Salvesen et al. (2009) did a similar study for the $\sim 10,000$ year old Cygnus Loop remnant, based on electron temperatures obtained from X-ray spectra as extracted from ROSAT-PSPC data. The shock velocity was determined from proper motion measurements taken from optical images obtained with the Palomar Observatory Sky Survey in two epochs, 39.1 years apart. From this study follows that the post-shock cosmic-ray pressure is negligible.

Helder et al. (2009) did a similar study on the TeV $\gamma$-ray remnant RCW 86 Aharonian et al. 2009) at the location where the shock front shows Xray synchrotron emission. They used the $\mathrm{H} \alpha$ line to determine the postshock proton temperature, and 2 epochs of Chandra data to measure the proper motion of the shock front. The shock velocity was significantly higher than one would expect from the post-shock proton temperature, resulting in $\beta=0.3$.

However, one has to caution that for the latter two studies, the proper motions were determined using emission from a different wavelength than the emission used to determine post-shock plasma temperature. This has the caveat that one might match physical parameters for shocks that happen to be in the same line of sight, but are not part of the same physical system.

Helder et al. (2010) measured the post-shock proton temperature as obtained with VLT/FORS2 for the LMC remnant SNR 0509-67.5 (Fig. 14). They combined this with a shock velocity determined from high resolution X-ray spectra as obtained with the XMM-Newton RGS instrument (Kosenko et al. 2008). The resulting shock velocities were $5000 \mathrm{~km} \mathrm{~s}^{-1}$ and $6000 \mathrm{~km} \mathrm{~s}^{-1}$ for the SW and NE shocks respectively. These velocities seem to be confirmed by results from a preliminary study carried out by Hovey and Hughes (2012), as they measure an average shock velocity of 6500 $\pm 200 \mathrm{~km} \mathrm{~s}^{-1}$ around the remnant, based on a proper motion study using two epochs of Hubble Space Telescope data. The velocities of Helder et al. (2010) resulted in a value of $\beta=0.7$ for the SW shock. The NE shock does not show evidence for cosmic-ray acceleration, but note that the uncertainty on the post-shock proton temperature is rather large, and therefore efficient cosmic-ray acceleration is not excluded either.

\subsubsection{Relation $w_{\mathrm{CR}}$ and $\epsilon_{\mathrm{CR}}$}

Equation 44 shows that measuring the fraction by which the temperature is lowered $\beta$ in itself does not provide information on the conditions of the cosmic-ray pressure of cosmic-ray escape of the shock. To solve this, Helder et al. (2009) and Helder et al. (2010) used a curve as an upper limit for $\epsilon_{\mathrm{CR}}$, leading to lower limits for $w_{\mathrm{CR}}$ :

$$
\frac{\epsilon_{\mathrm{CR}}}{w_{\mathrm{CR}}}=(1-1 / r)^{2} \text {. }
$$

This curve is based on the asymptotic particle spectra of a strong shock Malkov (1999). In a more recent study, Vink et al. (2010) calculated the 
relation between $\epsilon_{\mathrm{CR}}$ and $w_{\mathrm{CR}}$, using the standard Rankine-Hugoniot jump conditions over a shock, this time including a cosmic-ray precursor. Surprisingly, the $\epsilon_{\mathrm{CR}}-w_{\mathrm{CR}}$-relation resulting from this study overlaps exactly with the upper limit in equation 45 for a $\gamma$ of the CRs of $5 / 3$. For a $\gamma$ of the CRs of $4 / 3$, we get the following results: for $\mathrm{RCW} 86, w_{\mathrm{CR}}=0.5$ and $\epsilon_{\mathrm{CR}}=0.2$ and for the SW shock of SNR 0509-67.5, $w_{\mathrm{CR}}=0.21$ and $\epsilon_{\mathrm{CR}}=0.03$.

\section{Increased compression ratio}

As soon as a shock becomes efficient in accelerating, the density behind the shock front will increase for two reasons. The equation of state of the postshock plasma will soften, leading to a higher overall compression ratio. Also, if a shock loses its energy in the form of CRs escaping, it compensates for these energy losses by making the plasma behind the shock front denser, in order to maintain pressure equilibrium (equation 43 and Fig. 15). This immediately implies that the forward shock will be closer to the reverse shock and contact discontinuity than for a non-accelerating shock (Decourchelle et al. 2000, Ellison et al., 2004, Kosenko et al., 2011). The grey line in Fig. 15 shows the relation of the compression ratio as function of $\epsilon_{\mathrm{CR}}$.

This provides a way for measuring such an increased density. If one can accurately trace the outer edges of the shocked ejecta (i.e., the contact discontinuity), this can be compared with standard, non-CR accelerating models of supernova remnant evolution. This was done for the first time by Warren et al. (2005) for the Tycho supernova remnant using a principal component analysis to determine the location of the forward shock, contact discontinuity and reverse shock. Indeed, in Tycho, the contact discontinuity appears to be closer to the shock front than expected from standard models, implying efficient cosmic-ray acceleration (Kosenko et al., 2011).

In interpreting the outer edges of the ejecta in terms of the contact discontinuity, one should be careful. Hydrodynamical instabilities can bring the ejecta in front of the contact discontinuity (Blondin and Ellison, 2001; Wang 2011). Cassam-Chenaï et al. (2008) did a similar study on the SN1006 remnant, and revealed that the ejecta are closer to the forward shock at locations coinciding with X-ray synchrotron emission and $\mathrm{TeV} \gamma$-ray emission. However, they found the contact discontinuity to be too close to the shock front, for efficiently accelerating models, even for the regions that did not coincide with $\mathrm{TeV} \gamma$-ray emission of X-ray synchrotron emission. In a subsequent study, Miceli et al. (2009) found that the ejecta are less close to the shock front. Also, their study showed the ejecta to be similarly close to the shock front at the locations of the $\mathrm{TeV} \gamma$-ray emission as well as at other shocks. In fact, they concluded the shocks to be modified by cosmic-ray acceleration everywhere.

Note that the separation between shocked ejecta and the forward shocks might also be reduced if the forward shock encounters a density jump in the ambient medium (see e.g. Kosenko et al., 2010). 
4.1 Effect of CRs on Supernova Ejecta Morphology and Clumping

Blondin and Ellison (2001) carried out hydrodynamical simulations on the evolution of supernova remnants, including the effects of particle acceleration. In particular, they studied the effect of high compression ratios resulting from efficient cosmic-ray acceleration. They found a decrease in the distance between the forward and contact discontinuity with respect to a shock that was not accelerating particles. Moreover, in cases where particle acceleration is particularly efficient, instabilities at the contact discontinuity might reach the forward shock. This will cause the shock front to be perturbed by these instabilities. Rakowski et al. (2011) found protrusions along the eastern rim of SN 1006. This was interpreted in the context of an upstream magnetic field, amplified by streaming CRs Bell (2004, 2005), enhancing Rayleigh-Taylor instabilities post shock.

\subsection{Fast Moving Supernova Ejecta Fragments}

Aschenbach et al. (1995) discovered protruding structures in the Vela supernova remnant, using the ROSAT satellite and dubbed them 'shrapnels'. Miyata et al. (2001) used Chandra to investigate 'shrapnel A' and found that its silicon abundance is above solar. Cassiopeia A also has fragments of ejecta outside of the main rim. These were first detected in optical (e.g. van den Bergh, 1971; Kirshner and Chevalier, 1977, and the references therein). These fragments were interpreted in the context of shock-wave emission models by Raymond (1976), resulting in the conclusion that their abundances are consistent with uncontaminated material from the core of a massive star. Laming and Hwang (2003) analyzed several knots of Cassiopeia A as observed with Chandra.

\section{Molecular ion diagnostics of CR acceleration sources}

For a long time, the ionization of the interstellar medium by CRs has been considered an important factor in the phases of the interstellar medium (Field et al. 1969, Spitzer, 1978). The chemical reactions that take place in molecular clouds are determined by CR ionization rates (e.g. Herbst and Klemperer, 1973 Dalgarno, 2006). Padovani et al. (2009) calculated the gas ionization rates in molecular clouds assuming a CR spectrum extrapolated to low energies from observed CR proton and electron spectra. Hereby, they took into account CR particle energy loss processes. Their model agreed with observational data, only if the energy flux of the CR spectrum increases at low energies (below $\sim 100 \mathrm{MeV}$ ). The diffusion of CRs within molecular clouds are thought to be the dominated by the magnetic field orientation and strength. Padovani and Galli (2011) modeled this diffusion and found that magnetic mirroring is more important than magnetic focussing, resulting in a reduction of the CR ionization rate with a factor of about $2-3$ in the core of the molecular cloud. 
Molecular ion line observations may serve as a diagnostics of processes in CR acceleration sources. Near the IC443 supernova remnant, a $\mathrm{H}_{3}^{+}$column density of $3 \times 10^{14} \mathrm{~cm} \mathrm{~s}^{-1}$ was found in two sight lines (Indriolo et al., 2010). From these measurement, the ionization rate was found to exceed that of the average ionization rate of molecular clouds. However, the other four sight lines were consistent with typical Galactic values. A similar study on the W51C supernova remnant was carried out by Ceccarelli et al. (2011). Also for this remnant, the cloud ionization degree is highly enhanced, resulting in a CR ionization rate of $\sim 10^{-15} \mathrm{~s}^{-1}$. This is about 100 times the average value for molecular clouds.

Modeling of the ionization rate in interstellar material around a CR accelerating source shows that in infrared, the strongest lines of $\mathrm{H}_{3}^{+}$are between 3.0 and $4.6 \mu \mathrm{m}$ and $\mathrm{H}_{2}^{+}$lines of similar intensity are visible between 4.0 and $6.0 \mu \mathrm{m}$ Becker et al. (2011).

The Herschel key project "Chemical HErschel Surveys of Star forming region" (CHESS) (see e.g. Ceccarelli et al. 2010) collected high resolution molecular line spectra of star-forming regions that can be used to constrain the gas ionization rates in the molecular clouds. Also, Gerin et al. (2010) reported the detection of the ground state doublet of the methylidyne radical $\mathrm{CH}$ at $532 \mathrm{GHz}$ and $536 \mathrm{GHz}$ with the Herschel/HIFI instrument along the sight-line to the massive star-forming regions G10.6-0.4 (W31C), W49N, and W51 where some CR acceleration sources are residing.

\section{Particle acceleration in the Galactic Central regions}

The central 200 pc of the Galaxy are intense high energy emitters, from $\mathrm{X}$-rays to $\mathrm{TeV} \gamma$-rays. The physical conditions in these regions are indeed exceptional and make the Galactic Center (GC) a special place as regards particle acceleration. Bright non-thermal radio filaments trace magnetic fields of up to a $\mathrm{mG}$ (Ferrière, 2009) and large scale non-thermal emission suggest the average field is at least $50 \mu \mathrm{G}$ (Crocker et al. 2010). The Central Molecular Zone contains about 50 million solar masses of molecular gas (Morris and Serabyn, 1996) in which physical conditions are quite unusual, with high temperatures and a large velocity dispersion. The abundance and high density of the gas are such that intense star formation is taking place in this region. Three of the most massive young star clusters of the Galaxy lie within $30 \mathrm{pc}$ of the GC: the Arches, the Quintuplet and the central cluster. The Quintuplet harbors what could be the most massive star in the Galaxy: the Pistol star, a Luminous Blue Variable candidate with an estimated initial mass of $200 \mathrm{M}_{\odot}$ (Yungelson et al. 2008). The Arches cluster is one of the most massive and dense star clusters in the Galaxy. Its age is just 1-2 Myr and its central density reaches $10^{5} \mathrm{M}_{\odot} \mathrm{pc}^{-3}$ (Portegies Zwart et al. 2010 ). The inner $10 \mathrm{pc}$ are dominated by a bright supernova remnant, called Sgr A East. The radio shell is filled with unusually hot thermal X-ray emission and is consistent with a single star explosion about 10000 years ago. Finally at the very center, the faint source Sgr $A^{\star}$, is powered by a supermassive black hole of $410^{6} \mathrm{M}_{\odot}$ (Genzel et al. 2010, and references therein). The black hole is particularly quiet nowadays with a quiescent bolometric luminosity 
of about $10^{36} \mathrm{erg} / \mathrm{s}$ but there is good evidence that it has not always been so dim.

This exceptional environment and these extreme conditions make the GC region an interesting environment for testing particle acceleration models. After this very brief description of the scenery in the central 200 pc, we review some observational evidence for particle acceleration in the Galactic Central regions and discuss their possible origins. We discuss the observation of nonthermal emission from giant molecular clouds in the GC, first in non-thermal hard X-rays, possibly produced by sub-relativistic particles, and second in the $\mathrm{TeV}$ range, tracing an excess of very high energy CRs. Finally, we discuss the recent discovery of large bubbles of $\mathrm{GeV}$ emission extending above and below the GC.

\subsection{X-ray emission from GC molecular clouds}

One of the great legacies of the large modern X-ray observatories is the detailed mapping of the GC region. Among the main results, is the discovery that $\operatorname{Sgr} \mathrm{A}^{\star}$ is both remarkably faint nowadays with a quiescent luminosity of $10^{33} \mathrm{erg} / \mathrm{s}$ (Baganoff et al. 2003) and subject to frequent X-ray flares (Porquet et al., 2008), whose peak luminosities reach only a few $10^{35} \mathrm{erg} / \mathrm{s}$ or $10^{-9} \mathrm{~L}_{\mathrm{Edd}}$ for a $4 \times 10^{6} \mathrm{M}_{\odot}$ black hole. The surveys of the central 100 pc have also discovered that several massive molecular clouds in the GC display intense $\mathrm{Fe} \mathrm{K} \alpha 6.4 \mathrm{keV}$ line emission (Koyama et al., 1996, 2007) and non-thermal continuum (Sunyaev et al., 1993 : Revnivtsev et al. 2004 Bélanger et al., 2006). The origin of this emission has been strongly debated. The propagation of energetic charged particles inside the molecular clouds has been invoked as a possible explanation of the observed X-rays from the region.

Two distinct processes can produce hard X-ray continuum in combination with neutral iron line emission. First, low-energy CR electrons, with energies up to a few hundred $\mathrm{keV}$, diffusing into dense neutral matter will produce nonthermal bremsstrahlung and collisional K-shell ionization (Valinia et al. 2000). Second, subrelativistic ions propagating in the molecular cloud can radiate through inverse bremsstrahlung in the hard X-ray domain and create iron $\mathrm{K} \alpha$ vacancies (Dogiel et al. 2009 ). It is likely that the density of low energy CRs is larger in the central regions than in the rest of the Galaxy. The presence of strong non-thermal radio filaments in the vicinity of several molecular clouds makes it reasonable to consider the presence of a large density of CR electrons there. This is also supported, as noted by Yusef-Zadeh et al. (2007), by the observation of diffuse low-frequency radio emission in the central regions (LaRosa et al., 2005) and the high estimates of the ionization rate compared to the values obtained in the Galactic disk (Oka et al., 2005).

A possible mechanism to explain the production of such subrelativistic particles and the associated emission has been proposed by (Bykov, 2003. see section 4.2 in terms of fast moving knots resulting from supernova explosions.

Bykov (2002) has modeled this phenomenon taking into account injection, transport, first and second order Fermi acceleration as well as Coulomb losses 
which are dominant for low energy particles. The efficiency of bremsstrahlung radiation compared to ionization losses is more favorable in such enriched clumps which alleviates the energy requirement for such a process to take place. In particular, an O-dominated clump with an X-ray luminosity of $10^{31}$ $\mathrm{erg} / \mathrm{s}$ will lose $\sim 10^{35} \mathrm{erg} / \mathrm{s}$ through Coulomb losses (Bykov et al. 2008a).

Unfortunately, the recent observations of variability of the $6.4 \mathrm{keV}$ line emission in several distant clouds (Muno et al., 2007, Inui et al., 2009, Terrier et al. 2010) is contradicting the idea that CRs are producing the $6.4 \mathrm{keV}$ line emission. Besides, the observation of an apparent superluminal propagation of the $6.4 \mathrm{keV}$ emission in a molecular structure (Ponti et al., 2010) is also a clear sign that the clouds are in fact X-Ray Reflection Nebulae. Their emission is the result of Compton scattering and K-shell photo-ionization of neutral or low-ionized iron atoms by an intense X-ray radiation field generated in the GC. The required luminosity of illuminating the source is about $10^{39} \mathrm{erg} / \mathrm{s}$ making the supermassive black hole, Sgr $\mathrm{A}^{\star}$, the most likely candidate.

Not all Fe $\mathrm{K} \alpha$ emission is expected to be variable though, as is visible for instance on most clouds surrounding the Arches cluster region whose line flux have stayed constant over more than 8 years of XMM-Newton observations (Capelli et al., 2011). The best explanation for this (up-to-now) stationary emission is that it is produced by subrelativistic CR accelerated locally, possibly by collective effects in the cluster itself.

\subsection{An excess of very high energy CRs in the central $100 \mathrm{pc}$}

Besides the emission of the pulsar wind nebula G0.9+0.1 and the GC point source, the Cerenkov telescope system HESS has observed diffuse emission over $200 \mathrm{GeV}$ extending over the central degree. The spatial distribution of this emission is well correlated with that of interstellar matter which suggests that the very high energy photons are produced by CR ions interacting with dense clouds (Aharonian et al. 2006). The photon spectral index of this emission is 2.3, somewhat harder than the CR spectrum measured at Earth. This proves that the density of high energy CRs $(E>1 \mathrm{TeV})$ in the central $100 \mathrm{pc}$ is significantly larger than in a solar neighborhood. One or several CR accelerators must therefore be presently or very recently active there. Aharonian et al. (2006) have proposed that the supernova remnant Sgr A East is the source of these CRs. Taking into account the diffusion time of particles in the central regions, they showed that about $10 \%$ of the kinetic energy of the supernova remnant could explain the observed emission. Time dependent escape of energetic particles from supernova remnants has been proposed and studied by Gabici et al. (2009) and probably observed in systems such as W28 (Aharonian et al., 2008c).

The propagation of CRs in the complex environment of the GC is most likely quite complex. For instance, the large poloidal fields traced by nonthermal filaments might limit the diffusion of CRs in the Galactic plane. The idea of a single accelerator injecting CRs in the GC has therefore been questioned (Wommer et al., 2008, see for instance) and distributed turbulent acceleration in the inter-cloud medium has been proposed by several 
authors (Melia and Fatuzzo, 2011, Amano et al., 2011). We note that the massive young star clusters in the region can also provide significant particle acceleration through the collective effects of stellar winds and shocks. The interaction of the supersonic winds of the stars leads to the creation of a large superbubble filled with a hot and tenuous plasma. The propagation of supernova shocks inside the bubble create regions of enhanced turbulence where effective particle acceleration can take place (Bykov, 2001; Parizot et al., 2004, Higdon and Lingenfelter, 2005; Ferrand and Marcowith, 2010, this aspect is further discussed in the next section).

High-energy and very high energy $\gamma$-rays have been detected in regions spatially coincident with massive young clusters in the Galaxy: HESS has observed TeV photons possibly coming from the Westerlund 2 (Aharonian et al., 2007; HESS Collaboration et al. 2011b) and Westerlund 1 clusters (HESS Collaboration et al., 2011a). Very recently, an intense GeV emission pervading the Cygnus $\mathrm{X}$ region has also been found with Fermi (Ackermann et al. 2011). Although the nature and origin of these emission regions are not completely proven yet, they are arguing in favor of the collective effects inside massive star associations as effective particle accelerators. It is therefore very likely that the exceptional clusters at the GC are actively producing high energy particles, although future $\mathrm{TeV}$ observatories will be necessary to prove it. Thanks to their improved angular resolution compared to HESS they should be able to resolve the contribution of clusters to the diffuse GC TeV emission. We also note that the winds from the central cluster, containing two dozens of supergiant luminous and Wolf-Rayet stars as well as numerous $\mathrm{O}$ and B objects (Paumard et al., 2006), are most likely powering the accretion onto $\mathrm{Sgr} \mathrm{A}^{\star}$ and might also be at the origin of the strong point-like emission observed by HESS at the position of the GC (Quataert and Loeb, 2005).

\subsection{Large bubbles of $\gamma$-ray emission powered by the GC}

Recently, thanks to specific procedure of foreground subtraction with the Fermi data, Su et al. (2010) found a pair of large structures extending up to 50 degrees above and below the Galactic plane in the direction of the GC. These structures, called the "Fermi bubbles", are detected above a few $\mathrm{GeV}$ and have a hard spectrum extending up to $100 \mathrm{GeV}$. They seem to be associated to large scale structures observed in X-rays with ROSAT (BlandHawthorn and Cohen, 2003) and with an excess of radio emission found in WMAP data. At the distance of the GC, they have a luminosity of $4 \times 10^{37}$ erg/s and extend up to $10 \mathrm{kpc}$ outside of the Galactic disk. The estimated energy content of the bubbles is of the order of $10^{55}$ erg (Bland-Hawthorn and Cohen, 2003, Su et al., 2010).

The nature and the origin of the particles creating this $\gamma$-ray emission are still unclear. Inverse Compton emission of electrons requires only a fraction of the total energy in the form of energetic particles but requires in-situ acceleration to have very high energy electrons up to $10 \mathrm{kpc}$ above the disk. Energetic protons or nuclei have much longer lifetimes and can be injected from the Galactic central regions but require a much more substantial fraction of the total energy in the bubbles. 
The mechanism providing the large energy input is also quite uncertain. Su et al. (2010) discussed a possible starburst phase in the GC a few million years ago. Today, a more modest but constant injection of $10^{39} \mathrm{erg} / \mathrm{s}$ of CRs from the GC to the halo is inferred from the $\gamma$-ray emission from the central molecular zone and its infrared luminosity (Crocker et al., 2011). If this injection is integrated over a billion years, it might provide the correct energy budget (Crocker and Aharonian, 2011), although the particles have to be confined on extremely long timescales. Other mechanisms outline the possible role of the supermassive black hole. An intense AGN phase at Eddington luminosity accompanied by jets or outflows a few millions years ago has been invoked (Zubovas et al. 2011) as well as the recurrent (every $10^{4}-10^{5}$ years) accretion of stars captured by the black hole (Cheng et al., 2011).

A lot remains to be understood on the physics of these large $\gamma$-ray bubbles as well as on the processes producing the high energy emission in the molecular clouds of the central 200 pc. Nevertheless, they remind us that the GC plays a special role in the production of Galactic CRs.

\section{Alternatives for SNRs being main sources for GCRs}

\subsection{Particle acceleration in superbubbles}

Somewhere between $10^{17} \mathrm{eV}$ and $10^{19} \mathrm{eV}$, the CR origin is expected to transition from Galactic to extragalactic origin (e.g. Hillas, 2005; Aharonian et al. 2011). For this reason, Galactic cosmic-ray sources probably accelerate cosmic rays to above the knee. Important in models of CR acceleration is the environment in which the supernova evolves. Core collapse SNe usually reside in associations of young massive stars and molecular clouds. These associations usually create superbubbles i.e., large caverns filled mainly with a mixture of hot and warm gas produced by multiple supernova explosions. These superbubbles can have a significant effect on the structure of the disk and halo of the Galaxy (e.g. Norman and Ikeuchi, 1989, Heiles, 1990). Norman and Ikeuchi (1989) found that superbubbles during their outbursts create mass and energy flows from the disk into the halo and vice versa. The ongoing shocks marking the outer edges of a superbubbles are a plausible location of $\mathrm{CR}$ acceleration. Additionally, the material in the superbubbles possibly has an enriched composition with Wolf-Rayet winds and SN ejecta (e.g. Bykov and Fleishman, 1992, Axford, 1994, Klepach et al., 2000, Bykov, 2001; Binns et al. $\mid 2007$; Ferrand and Marcowith, 2010).

Observational studies on OB-associations and young globular cluster have been carried out both in the Galaxy and the Large Magellanic Cloud. A detailed study on the Galactic OB-association Cygnus OB-2 by Knödlseder (2000) showed that it has $2600 \pm 400$ OB members, out of which $120 \pm$ 20 are $\mathrm{O}$ stars. The corresponding high number of X-ray sources (Wright and Drake, 2009) suggests that Cygnus OB2 is one of the most massive star forming regions in the Galaxy. Given its relative small size, it is inevitable that the region contains strong winds closely together, accumulating in strong shocks. These are the regions that are particularly expected to be particle accelerators (e.g. Eichler and Usov, 1993, Torres et al., 2004). 
Indeed, superbubble 30 Dor C in the Large Magellanic Cloud appears to emit non-thermal X-rays (Bamba et al., 2004). The shape of this non-thermal emission, coincides with radio emission from this region: nearly circular shell with a radius of $40 \mathrm{pc}$. Actually, this picture resembles that of the outer rims of young supernova remnants. One should realize that the life time of superbubbles exceeds that of supernova remnants by a large factor, resulting in a particle acceleration time that is substantially longer than that of supernova remnants.

Also the OB association LH9 located in the HII complex N11 in the Large Magellanic Could shows evidence for non-thermal X-ray emission Maddox et al. (2009). Interestingly, the kinetic and thermal energy of LH9 is only half of the energy mechanical energy transferred into the $\mathrm{OB}$ association by its stars. This is consistent with current models of CR acceleration by superbubbles (e.g. Bykov, 2001). Overall, we would expect a galaxy with a high star formation rate to have a higher cosmic-ray energy density than a galaxy with an average star formation rate. Indeed, the starburst galaxy NGC 253 has been detected by the HESS telescope (Acero et al. 2009a). Also, Ackermann et al. (2011) detected the star-forming region Cygnus $\mathrm{X}$ in $\mathrm{GeV} \gamma$-rays.

The CR Isotope Spectrometer aboard the Advanced Composition Explorer spacecraft found that the isotopic abundances of the CR spectrum is consistent with solar, with a $20 \%$ contribution of Wolf-Rayet material. Since Wolf-Rayet stars are stages in the evolution of OB stars, that largely reside in B associations and superbubbles, this supports the idea that a substantial fraction of the Galactic CRs are accelerated in superbubbles.

The efficiency with which kinetic energy in superbubbles can be transferred into CR energy are estimated to be around 20\% (Bykov, 2001). Superbubbles are surrounded by massive shells and therefore expand much slower than supernova remnants reducing the adiabatic deceleration and alleviating the CR escape from superbubbles. Magnetic fields inside the superbubble should be amplified up to about $30 \mu \mathrm{G}$ to provide the maximal energies of $\mathrm{CR}$ protons to be about $10^{8} \mathrm{GeV}$ and higher for heavy $\mathrm{CR}$ nuclei that will dominate the accelerated $\mathrm{CR}$ composition at the highest energies in the model. We illustrate in Figure 16 the CR composition simulated in a model where CRs of energies above the knee are accelerated in a superbubble (see Bykov and Toptygin, 2001).

\subsection{Reaching and passing the knee}

A decade ago, the magnetic field strength in supernova remnants was believed to be not more than four times Galactic magnetic field as this is the compression factor of a non-accelerating shock. This implies a rather large diffusion coefficient for particles that are being accelerated and therewith a relative large time before the particles return over the shock front. Lagage and Cesarsky (1983) were the first to calculate that with these high diffusion coefficients, supernova remnants are not capable of accelerating particles up to the knee in the CR spectrum (see also section 2.3). 


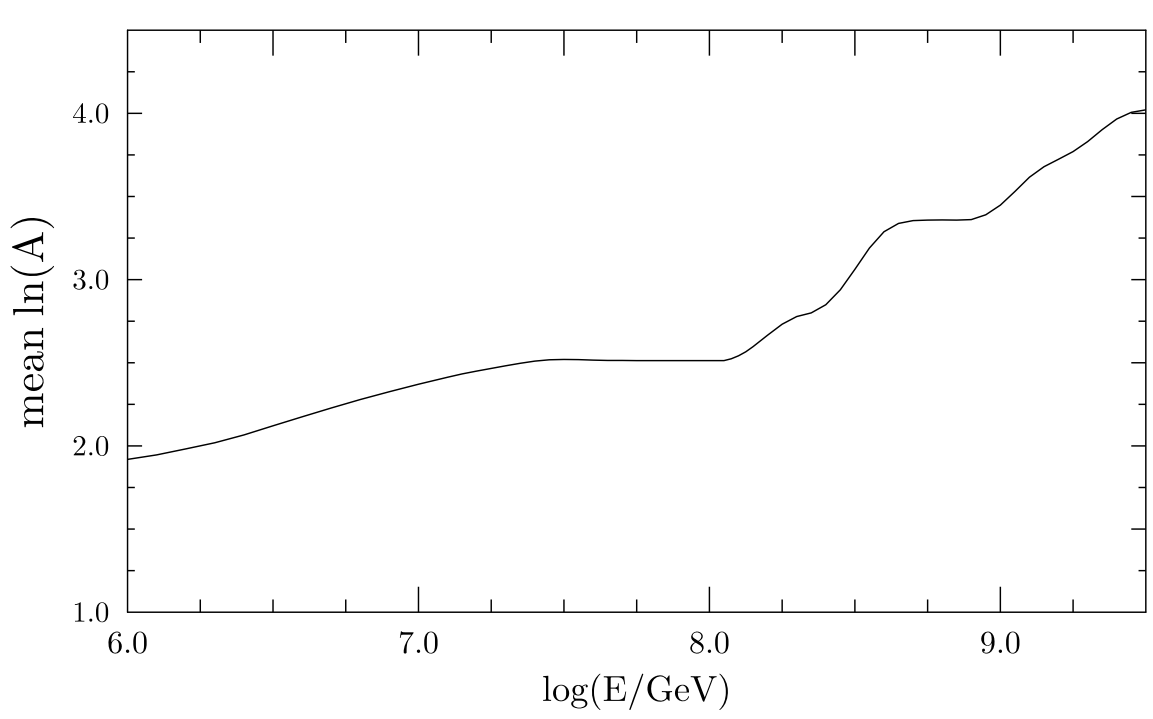

Fig. $16 \mathrm{CR}$ composition above the knee simulated in a model of CR acceleration in a superbubble (adapted from Bykov and Toptygin, 2001). The energy dependence of the mean logarithm of atomic mass InA of accelerated particles is shown.

Bell and Lucek (2001) calculated that CRs, as they stream up and down the shock front, can amplify the magnetic fields themselves by even an order of magnitude. This has observationally been confirmed (Vink and Laming, 2003) and has now been found for a substantial amount of supernova remnants (Völk et al., 2005, and Table 3). This shows that from a theoretical perspective, supernova remnants should be able to accelerate particles up to the knee.

Spectra of X-ray synchrotron emitting remnants have shown that the cutoff of the electron spectrum is at $\mathrm{TeV}$ energies (Parizot et al., 2006). This is still a few orders of magnitude short of the knee, but we know that the maximum energies of electrons are limited by the energy they lose as they emit synchrotron emission. Protons do not suffer from these losses and might well reach higher energies.

Pion decay $\gamma$-rays are produced by protons in a more or less direct fashion. Therefore, observing supernova remnants with current $\mathrm{GeV}$ and $\mathrm{TeV} \gamma$-ray telescopes would yield a wealth of information on their CR proton content. However, for some remnants, it is debated whether to interpret the $\mathrm{TeV} \gamma$ ray data in terms of Inverse Compton scattering (which would be electrons again) or pion decay. In any case, even if pion decay would be the correct model, none of the remnants observed thus far show evidence for protons with energies up to $3 \times 10^{15} \mathrm{eV}$.

There is, however indirect evidence for protons with these high energies in supernova remnants, as shown by the stripes of non-thermal X-ray emission on the surface of Tycho Eriksen et al. (2011); Bykov et al. (2011a). Moreover, (Ptuskin et al. , 2010) found that remnants of type IIb supernovae should be able to accelerate Fe ions up to $5 \times 10^{18} \mathrm{eV}$ and Morlino and Caprioli (2011) 
modeled the broadband emission of Tycho's supernova remnant and found that protons were at least accelerated up to $5 \times 10^{14} \mathrm{eV}$.

\section{Summary}

We reviewed the current observational research on the sources of Galactic cosmic rays. We described current shock acceleration theory, and how nonlinear shock acceleration modifies the shock structure as well as the emission mechanisms that reveal the presence of cosmic rays. We use this knowledge to review the current observational status of cosmic-ray acceleration in supernova remnants, the Galactic center region and superbubbles.

In these concluding remarks, we briefly point out some of the remaining open issues related to high Mach number shocks of supernova remnants being the main sources for Galactic cosmic rays. One of the big uncertainties in present-day cosmic-ray acceleration theory is the physics that determines the amount of particles getting injected into the acceleration process. This is particularly important, as it is one of the key ingredients for determining the efficiency of shocks accelerating cosmic rays.

Also, the maximal energy to which cosmic-rays can be accelerated by supernova remnants is unclear. The discovery of magnetic field amplification makes acceleration up to, or beyond the knee, now much more plausible than was thought three decades ago Lagage and Cesarsky (1983), but observation proof for the presence of particles with energies in excess of $10^{15} \mathrm{eV}$ in SNRs is still lacking. Third, we would like to point out that the moment at which the bulk of the cosmic rays escape the remnant still unclear is. In this context, it is useful to point out that most of the remnants detected in $\gamma$-rays are mature remnants, interacting with a molecular cloud, suggesting that these remnants still have a substantial cosmic-ray content at older ages. However, the maximum energies of the primary cosmic-ray particles seem in these mature SNRs limited to at best 1-10 GeV.

In the near future more results are expected from the current generation of X-ray, and $\gamma$-ray telescopes. But future telescopes such as the Cherenkov Telescope Array, will greatly improve the sensitivity which gamma-rays can be detected. In addition, our knowledge of high Mach number shocks in general, and the role of cosmic rays for their thermodynamics, will improve with future X-ray and optical spectroscopic observations, such as with the Japanese X-ray telescope Astro-H and 30m class optical telescopes such as ELT.

These open issues will most efficiently be solved if theory and observations work hand-in-hand. Workshops as this in Bern, provide indispensable opportunities for close interaction between theorist and observers.

\section{Acknowledgements}

The authors thank the ISSI in Bern for their hospitality and for organizing the workshop that resulted in this chapter. This work has been supported by 
SAO grant GO0-11072X (E.A.H.). A.M.B. was supported in part by the Russian government grant 11.G34.31.0001 to Sankt-Petersburg State Politechnical University, and also by the RAS Presidium Program and by the RFBR grant 11-02-12082. He performed the simulations at the Joint Supercomputing Center (JSCC RAS) and the Supercomputing Center at Ioffe Institute, St. Petersburg.

\section{References}

Abdo AA, Fermi LAT Collaboration (2011) Observations of the young supernova remnant RX J1713.7-3946 with the Fermi Large Area Telescope. ArXiv e-prints 1103.5727

Abdo AA, et al. (2009) Fermi LAT Discovery of Extended Gamma-Ray Emission in the Direction of Supernova Remnant W51C. ApJ706:L1-L6, 0910.0908

Abdo AA, et al. (2010a) Fermi Large Area Telescope Observations of the Supernova Remnant W28 (G6.4-0.1). ApJ718:348-356

Abdo AA, et al. (2010b) Fermi-Lat Discovery of GeV Gamma-Ray Emission from the Young Supernova Remnant Cassiopeia A. ApJ710:L92-L97, 1001.1419

Abdo AA, et al. (2010c) Fermi-LAT Study of Gamma-ray Emission in the Direction of Supernova Remnant W49B. ApJ722:1303-1311

Abdo AA, et al. (2010d) Gamma-Ray Emission from the Shell of Supernova Remnant W44 Revealed by the Fermi LAT. Science 327:1103-

Abdo AA, et al. (2010e) Observation of Supernova Remnant IC 443 with the Fermi Large Area Telescope. ApJ712:459-468, 1002.2198

Acciari VA, Aliu E, Arlen T, Aune T, et al. (2009) Observation of Extended Very High Energy Emission from the Supernova Remnant IC 443 with VERITAS. ApJ698:L133-L137, 0905.3291

Acciari VA, Aliu E, Arlen T, Aune T, et al. (2011) Discovery of TeV Gammaray Emission from Tycho's Supernova Remnant. ApJ730:L20+, 1102.3871

Acciari VA, et al. (2010) Observations of the Shell-type Supernova Remnant Cassiopeia A at TeV Energies with VERITAS. ApJ714:163-169, 1002. 2974

Acero F, Aharonian F, Akhperjanian AG, Anton G, et al. (2009a) Detection of Gamma Rays from a Starburst Galaxy. Science 326:1080-, 0909.4651

Acero F, Ballet J, Decourchelle A (2007) The gas density around SN 1006. A\&A475:883-890, 0709.0956

Acero F, Ballet J, Decourchelle A, Lemoine-Goumard M, et al. (2009b) A joint spectro-imaging analysis of the XMM-Newton and HESS observations of the supernova remnant RX J1713.7-3946. A\&A505:157-167, 0906.1073

Acero F, et al. (2010) First detection of VHE $\gamma$-rays from SN 1006 by HESS. A\&A516:A62+

Achterberg A, Blandford RD, Reynolds SP (1994) Evidence for enhanced MHD turbulence outside sharp-rimmed supernova remnants. A\&A281:220-230 
Ackermann M, Ajello M, Allafort A, et al (2011) A cocoon of freshly accelerated cosmic rays detected by Fermi in the Cygnus Superbubble. Science 334:1103-1107

Aharonian A F Akhperjanian, Barrio J, et al. (2001) Evidence for $\mathrm{TeV}$ gamma ray emission from Cassiopeia A. A\&A370:112-120

Aharonian F, Akhperjanian AG, Barres de Almeida U, Bazer-Bachi AR, et al. (2008a) Discovery of a VHE gamma-ray source coincident with the supernova remnant CTB 37A. A\&A490:685-693, 0803.0702

Aharonian F, Akhperjanian AG, Barres de Almeida U, Bazer-Bachi AR, et al. (2008b) Exploring a SNR/molecular cloud association within HESS J1745-303. A\&A483:509-517, 0803.2844

Aharonian F, Akhperjanian AG, Bazer-Bachi AR, Behera B, et al. (2008c) Discovery of very high energy gamma-ray emission coincident with molecular clouds in the W 28 (G6.4-0.1) field. A\&A481:401-410, 0801.3555

Aharonian F, Akhperjanian AG, Bazer-Bachi AR, Beilicke M, et al. (2006) Discovery of very-high-energy $\gamma$-rays from the Galactic Centre ridge. Nature439:695-698, arXiv:astro-ph/0603021

Aharonian F, Akhperjanian AG, Bazer-Bachi AR, Beilicke M, et al. (2007) Detection of extended very-high-energy $\gamma$-ray emission towards the young stellar cluster Westerlund 2. A\&A467:1075-1080, arXiv:astro-ph/ 0703427

Aharonian F, Bykov A, Parizot E, Ptuskin V, et al. (2011) Cosmic Rays in Galactic and Extragalactic Magnetic Fields. Space Sci. Rev.:2681105.0131

Aharonian F, et al. (2005) Detection of $\mathrm{TeV} \gamma$-ray emission from the shelltype supernova remnant RX J0852.0-4622 with HESS. A\&A437:L7-L10

Aharonian F, et al. (2009) Discovery of Gamma-Ray Emission From the Shell-Type Supernova Remnant RCW 86 With Hess. ApJ692:1500-1505

Aharonian FA, Atoyan AM (1996) On the emissivity of $\pi^{\wedge} 0^{\wedge}$-decay gamma radiation in the vicinity of accelerators of galactic cosmic rays. A\&A309:917-928

Aharonian FA, Atoyan AM (1999) On the origin of TeV radiation of SN 1006. A\&A351:330-340

Aharonian FA, Atoyan AM (2000) Broad-band diffuse gamma ray emission of the galactic disk. A\&A362:937-952, arXiv:astro-ph/0009009

Aharonian FA, et al. (2004) High-energy particle acceleration in the shell of a supernova remnant. Nature432:75-77, arXiv : astro-ph/0411533

Albert J, et al. (2007a) Discovery of Very High Energy Gamma Radiation from IC 443 with the MAGIC Telescope. ApJ664:L87-L90, 0705.3119

Albert J, et al. (2007b) Observation of VHE $\gamma$-rays from Cassiopeia A with the MAGIC telescope. A\&A474:937-940, arXiv:0706.4065

Allen GE, Gotthelf EV, Petre R (1999) Evidence of 10-tev to 100-tev electrons in supernova remnants. in the proceedings of the 26th International Cosmic Ray Conference (astro-ph/9908209) astro-ph/9908209

Allen GE, Houck JC, Sturner SJ (2008) Evidence of a Curved Synchrotron Spectrum in the Supernova Remnant SN 1006. ApJ683:773-785, 0807. 1702

Allen GE, et al. (1997) Evidence of X-Ray Synchrotron Emission from Electrons Accelerated to $40 \mathrm{TeV}$ in the Supernova Remnant Cassiopeia A. 
ApJ487:L97-L100

Amano T, Torii K, Hayakawa T, Fukui Y (2011) Stochastic Acceleration of Cosmic Rays in the Central Molecular Zone of the Galaxy. ArXiv e-prints 1110.3140

Amato E, Blasi P (2006) Non-linear particle acceleration at nonrelativistic shock waves in the presence of self-generated turbulence. MNRAS371:1251-1258, arXiv:astro-ph/0606592

Aschenbach B, Egger R, Trümper J (1995) Discovery of explosion fragments outside the Vela supernova remnant shock-wave boundary. Nature373:587590

Asvarov AI, Guseinov OH, Kasumov FK, Dogel' VA (1990) The hard X-ray emission of the young supernova remnants. A\&A229:196-200

Atwood WB, Abdo AA, Ackermann M, Althouse W, et al. (2009) The Large Area Telescope on the Fermi Gamma-Ray Space Telescope Mission. ApJ697:1071-1102, 0902.1089

Axford WI (1994) The origins of high-energy cosmic rays. ApJS90:937-944

Axford WI, Leer E, Skadron G (1977) The acceleration of cosmic rays by shock waves. In: International Cosmic Ray Conference, vol. 11 of International Cosmic Ray Conference, pp. 132-137

Baade W, Zwicky F (1934) Cosmic Rays from Super-novae. Proceedings of the National Academy of Science 20:259-263

Baars JWM, Genzel R, Pauliny-Toth IIK, Witzel A (1977) The absolute spectrum of CAS A - an accurate flux density scale and a set of secondary calibrators. A\&A61:99-106

Badenes C, Borkowski KJ, Hughes JP, Hwang U, et al. (2006) Constraints on the Physics of Type Ia Supernovae from the X-Ray Spectrum of the Tycho Supernova Remnant. ApJ645:1373-1391, arXiv: astro-ph/0511140

Baganoff FK, Maeda Y, Morris M, Bautz MW, et al. (2003) Chandra X-Ray Spectroscopic Imaging of Sagittarius $A^{*}$ and the Central Parsec of the Galaxy. ApJ591:891-915, arXiv:astro-ph/0102151

Ballet J (2006) X-ray synchrotron emission from supernova remnants. Advances in Space Research 37:1902-1908, arXiv:astro-ph/0503309

Bamba A, Koyama K, Tomida H (2000) Discovery of non-thermal X-rays from the shell of RCW 86. PASJ52:1157-1163

Bamba A, Ueno M, Nakajima H, Koyama K (2004) Thermal and Nonthermal X-Rays from the Large Magellanic Cloud Superbubble 30 Doradus C. ApJ602:257-263

Bamba A, Yamazaki R, Yoshida T, Terasawa T, et al. (2005) A Spatial and Spectral Study of Nonthermal Filaments in Historical Supernova Remnants: Observational Results with Chandra. ApJ621:793-802, arXiv: astro-ph/0411326

Bandiera R, Petruk O (2004) Analytic solutions for the evolution of radiative supernova remnants. A\&A419:419-423, arXiv:astro-ph/0402598

Barbon R, Ciatti F, Rosino L (1979) Photometric properties of type II supernovae. A\&A72:287-292

Bartel N (2009) Supernova VLBI. In: Approaching Micro-Arcsecond Resolution with VSOP-2: Astrophysics and Technologies (ed. Y Hagiwara, E Fomalont, M Tsuboi, \& M Yasuhiro), vol. 402 of Astronomical Society 
of the Pacific Conference Series, pp. 243-+

Becker JK, Black JH, Safarzadeh M, Schuppan F (2011) Tracing the Sources of Cosmic Rays with Molecular Ions. ApJ739:L43+, 1106.4740

Bélanger G, Goldwurm A, Renaud M, Terrier R, et al. (2006) A Persistent High-Energy Flux from the Heart of the Milky Way: INTEGRAL's View of the Galactic Center. ApJ636:275-289, arXiv:astro-ph/0508128

Bell AR (1978) The acceleration of cosmic rays in shock fronts. II. MNRAS182:443-455

Bell AR (2004) Turbulent amplification of magnetic field and diffusive shock acceleration of cosmic rays. MNRAS353:550-558

Bell AR (2005) The interaction of cosmic rays and magnetized plasma. MNRAS358:181-187

Bell AR, Lucek SG (2001) Cosmic ray acceleration to very high energy through the non-linear amplification by cosmic rays of the seed magnetic field. MNRAS321:433-438

Berezhko EG, Ellison DC (1999) A Simple Model of Nonlinear Diffusive Shock Acceleration. ApJ526:385-399

Berezhko EG, Ksenofontov LT, Ptuskin VS, Zirakashvili VN, et al. (2003a) Cosmic ray production in supernova remnants including reacceleration: The secondary to primary ratio. A\&A410:189-198

Berezhko EG, Ksenofontov LT, Völk HJ (2003b) Confirmation of strong magnetic field amplification and nuclear cosmic ray acceleration in SN 1006. A\&A412:L11-L14

Berezhko EG, Völk HJ (2008) Hadronic versus leptonic origin of the gammaray emission from supernova remnant RX J1713.7-3946. A\&A492:695-701, 0810.0988

Berezhko EG, Völk HJ (2010) Nonthermal and thermal emission from the supernova remnant RX J1713.7-3946. A\&A511:A34+, 0910.2094

Binns WR, Wiedenbeck ME, Arnould M, Cummings AC, et al. (2007) OB Associations, Wolf Rayet Stars, and the Origin of Galactic Cosmic Rays. Space Sci. Rev.130:439-449, 0707.4645

Bisnovatyi-Kogan GS, Silich SA (1995) Shock-wave propagation in the nonuniform interstellar medium. Reviews of Modern Physics 67:661-712

Bland-Hawthorn J, Cohen M (2003) The Large-Scale Bipolar Wind in the Galactic Center. ApJ582:246-256, arXiv:astro-ph/0208553

Blandford R, Eichler D (1987) Particle acceleration at astrophysical shocks: A theory of cosmic ray origin. Phys. Rep.154:1-75

Blandford RD, Ostriker JP (1978) Particle acceleration by astrophysical shocks. ApJ221:L29-L32

Blasi P, Gabici S, Vannoni G (2005) On the role of injection in kinetic approaches to non-linear particle acceleration at non-relativistic shock waves. MNRAS361:907-918, arXiv:astro-ph/0505351

Bleeker JAM, et al. (2001) Cassiopeia A: On the origin of the hard X-ray continuum and the implication of the observed O VIII Ly- $\alpha / \mathrm{Ly}-\beta$ distribution. A\&A365:L225-L230

Blondin JM, Ellison DC (2001) Rayleigh-Taylor Instabilities in Young Supernova Remnants Undergoing Efficient Particle Acceleration. ApJ560:244253 , arXiv: astro-ph/0104024 
Borkowski KJ, Reynolds SP, Green DA, Hwang U, et al. (2010) Radioactive Scandium in the Youngest Galactic Supernova Remnant G1.9+0.3. ApJ724:L161-L165, 1006.3552

Borkowski KJ, Rho J, Reynolds SP, Dyer KK (2001) Thermal and nonthermal X-Ray emission in supernova remnant RCW 86. ApJ550:334-345

Boulares A, Cox DP (1988) Application of cosmic-ray shock theories to the Cygnus Loop - an alternative model. ApJ333:198-218

Brun F, de Naurois M, Hofmann W, Carrigan S, et al. (2011) Discovery of VHE gamma-ray emission from the W49 region with H.E.S.S. ArXiv e-prints 1104.5003

Butt YM, Porter TA, Katz B, Waxman E (2008) X-ray hotspot flares and implications for cosmic ray acceleration and magnetic field amplification in supernova remnants. MNRAS386:L20-L22, 0801.4954

Bykov AM (2001) Particle Acceleration and Nonthermal Phenomena in Superbubbles. Space Sci. Rev.99:317-326

Bykov AM (2002) X-ray line emission from supernova ejecta fragments. A\&A390:327-335, arXiv:astro-ph/0205225

Bykov AM (2003) Faint hard X-ray sources in the Galactic Center region: Supernova ejecta fragments population. A\&A410:L5-L8, arXiv: astro-ph/0309234

Bykov AM (2004) Shocks and particle acceleration in SNRs: theoretical aspects. Advances in Space Research 33:366-375

Bykov AM, Ellison DC, Osipov SM, Pavlov GG, et al. (2011a) X-ray Stripes in Tycho's Supernova Remnant: Synchrotron Footprints of a Nonlinear Cosmic-ray-driven Instability. ApJ735:L40+, 1106.3441

Bykov AM, Fleishman GD (1992) On non-thermal particle generation in superbubbles. MNRAS255:269-275

Bykov AM, Krassilchtchikov AM, Uvarov YA, Bloemen H, et al. (2008a) Isolated X-Ray-Infrared Sources in the Region of Interaction of the Supernova Remnant IC 443 with a Molecular Cloud. ApJ676:1050-1063, 0801.1255

Bykov AM, Osipov SM, Ellison DC (2011b) Cosmic ray current driven turbulence in shocks with efficient particle acceleration: the oblique, longwavelength mode instability. MNRAS410:39-52, 1010.0408

Bykov AM, Toptygin IN (2001) A Model of Particle Acceleration to High Energies by Multiple Supernova Explosions in OB Associations. Astronomy Letters 27:625-633

Bykov AM, Uvarov YA (1999) Electron kinetics in collisionless shock waves. JETP 88:465-475

Bykov AM, Uvarov YA, Bloemen JBGM, den Herder JW, et al. (2009) A model of polarized X-ray emission from twinkling synchrotron supernova shells. MNRAS399:1119-1125, 0907.2521

Bykov AM, Uvarov YA, Ellison DC (2008b) Dots, Clumps, and Filaments: The Intermittent Images of Synchrotron Emission in Random Magnetic Fields of Young Supernova Remnants. ApJ689:L133-L136, 0811.2498

Capelli R, Warwick RS, Porquet D, Gillessen S, et al. (2011) Fe K $\alpha$ line emission from the Arches cluster region - evidence for ongoing particle bombardment? A\&A530:A38+, 1104.2039 
Caprioli D, Amato E, Blasi P (2010) The contribution of supernova remnants to the galactic cosmic ray spectrum. Astroparticle Physics 33:160-168, 0912.2964

Carlton AK, Borkowski KJ, Reynolds SP, Hwang U, et al. (2011) Expansion of the Youngest Galactic Supernova Remnant G1.9+0.3. ArXiv e-prints 1106.4498

Cassam-Chenaï G, Hughes JP, Ballet J, Decourchelle A (2007) The Blast Wave of Tycho's Supernova Remnant. ApJ665:315-340, arXiv:astro-ph/ 0703239

Cassam-Chenaï G, Hughes JP, Reynoso EM, Badenes C, et al. (2008) Morphological Evidence for Azimuthal Variations of the Cosmic-Ray Ion Acceleration at the Blast Wave of SN 1006. ApJ680:1180-1197, arXiv: 0803.0805

Castro D, Slane P (2010) Fermi Large Area Telescope Observations of Supernova Remnants Interacting with Molecular Clouds. ApJ717:372-378, 1002.2738

Ceccarelli C, Bacmann A, Boogert A, Caux E, et al. (2010) Herschel spectral surveys of star-forming regions. Overview of the $555-636 \mathrm{GHz}$ range. A\&A521:L22+

Ceccarelli C, Hily-Blant P, Montmerle T, Dubus G, et al. (2011) Supernovaenhanced Cosmic-Ray Ionization and Induced Chemistry in a Molecular Cloud of W51C. ApJ740:L4, 1108.3600

Cheng KS, Chernyshov DO, Dogiel VA, Ko CM, et al. (2011) Origin of the Fermi Bubble. ApJ731:L17, 1103.1002

Chevalier RA (1982a) Self-similar solutions for the interaction of stellar ejecta with an external medium. ApJ258:790-797

Chevalier RA (1982b) The radio and X-ray emission from type II supernovae. ApJ259:302-310

Chevalier RA (1983) Blast waves with cosmic-ray pressure. ApJ272:765-772

Chevalier RA, Kirshner RP, Raymond JC (1980) The optical emission from a fast shock wave with application to supernova remnants. ApJ235:186-195

Chevalier RA, Oishi J (2003) Cassiopeia A and Its Clumpy Presupernova Wind. ApJ593:L23-L26

Chevalier RA, Raymond JC (1978) Optical emission from a fast shock wave - The remnants of Tycho's supernova and SN 1006. ApJ225:L27-L30

Cox DP, Shelton RL, Maciejewski W, Smith RK, et al. (1999) Modeling W44 as a Supernova Remnant in a Density Gradient with a Partially Formed Dense Shell and Thermal Conduction in the Hot Interior. I. The Analytical Model. ApJ524:179-191

Crocker RM, Aharonian F (2011) Fermi Bubbles: Giant, Multibillion-YearOld Reservoirs of Galactic Center Cosmic Rays. Physical Review Letters 106(10):101102, 1008.2658

Crocker RM, Jones DI, Aharonian F, Law CJ, et al. (2011) $\gamma$-rays and the far-infrared-radio continuum correlation reveal a powerful Galactic Centre wind. MNRAS411:L11-L15, 1009.4340

Crocker RM, Jones DI, Melia F, Ott J, et al. (2010) A lower limit of 50 microgauss for the magnetic field near the Galactic Centre. Nature463:65$67,1001.1275$ 
CTA Consortium T (2010) Design Concepts for the Cherenkov Telescope Array. ArXiv e-prints 1008.3703

Dalgarno A (2006) Interstellar Chemistry Special Feature: The galactic cosmic ray ionization rate. Proceedings of the National Academy of Science 1031:12 269-12 273

de Laat APLS (2011) Balmer-Dominated Shocks and the effects of CosmicRay acceleration. Master's thesis, Utrecht University

Decourchelle A, Ellison DC, Ballet J (2000) Thermal X-Ray Emission and Cosmic-Ray Production in Young Supernova Remnants. ApJ543:L57-L60, arXiv:astro-ph/0008344

DeLaney T, Koralesky B, Rudnick L, Dickel JR (2002) Radio Spectral Index Variations and Physical Conditions in Kepler's Supernova Remnant. ApJ580:914-927, arXiv:astro-ph/0210355

Delaney T, Rudnick L (2003) The first measurement of Cassiopeia A's forward shock expansion rate. ApJ589:818

DeLaney T, Rudnick L, Fesen RA, Jones TW, et al. (2004) Kinematics of X-Ray-Emitting Components in Cassiopeia A. ApJ613:343-348, arXiv: astro-ph/0406045

Dermer CD (1986) Binary collision rates of relativistic thermal plasmas. II Spectra. ApJ307:47-59

Dickel JR, Milne DK (1976) Magnetic fields in supernova remnants. Australian Journal of Physics 29:435-460

Dogiel V, Cheng KS, Chernyshov D, Bamba A, et al. (2009) Origin of $6.4 \mathrm{keV}$ Line Emission from Molecular Clouds in the Galactic Center. PASJ61:901-

Draine BT, McKee CF (1993) Theory of interstellar shocks. ARA\&A31:373 432

Drury L, Aharonian FA, Malyshev D, Gabici S (2009) On the plasma temperature in supernova remnants with cosmic-ray modified shocks. A\&A496:1$6,0811.3566$

Drury LO (1983) An introduction to the theory of diffusive shock acceleration of energetic particles in tenuous plasmas. Reports on Progress in Physics 46:973-1027

Drury LO (2011) Escaping the accelerator: how, when and in what numbers do cosmic rays get out of supernova remnants? MNRAS415:1807-1814, 1009.4799

Drury LO, Duffy P, Kirk JG (1996) Limits on diffusive shock acceleration in dense and incompletely ionised media. A\&A309:1002-1010, arXiv: astro-ph/9510066

Drury LO, Voelk JH (1981) Hydromagnetic shock structure in the presence of cosmic rays. ApJ248:344-351

Dubner G, Giacani E, Reynoso E, Parón S (2004) The molecular clouds in the environs of the supernova remnants G349.7+0.2 and G18.8+0.3. A\&A426:201-212

Dwarkadas VV, Chevalier RA (1998) Interaction of Type IA Supernovae with Their Surroundings. ApJ497:807-+

Eichler D, Usov V (1993) Particle acceleration and nonthermal radio emission in binaries of early-type stars. ApJ402:271-279 
Ellison DC, Decourchelle A, Ballet J (2004) Hydrodynamic simulation of supernova remnants including efficient particle acceleration. A\&A413:189 201, arXiv:astro-ph/0308308

Ellison DC, Decourchelle A, Ballet J (2005) Nonlinear particle acceleration at reverse shocks in supernova remnants. A\&A429:569-580, arXiv: astro-ph/0409182

Ellison DC, Patnaude DJ, Slane P, Raymond J (2010) Efficient Cosmic Ray Acceleration, Hydrodynamics, and Self-Consistent Thermal X-Ray Emission Applied to Supernova Remnant RX J1713.7-3946. ApJ712:287-293, 1001.1932

Eriksen KA, Hughes JP, Badenes C, Fesen R, et al. (2011) Evidence for Particle Acceleration to the Knee of the Cosmic Ray Spectrum in Tycho's Supernova Remnant. ApJ728:L28+, 1101.1454

Esposito JA, Hunter SD, Kanbach G, Sreekumar P (1996) EGRET Observations of Radio-bright Supernova Remnants. ApJ461:820

Favata F, et al. (1997) The broad-band X-ray spectrum of the Cas A supernova remnant as seen by the BeppoSAX observatory. A\&A324:L49-L52

Ferrand G, Marcowith A (2010) On the shape of the spectrum of cosmic rays accelerated inside superbubbles. A\&A510:A101+, 0911.4457

Ferrière K (2009) Interstellar magnetic fields in the Galactic center region. A\&A505:1183-1198, 0908.2037

Fesen RA, Hammell MC, Morse J, Chevalier RA, et al. (2006) The Expansion Asymmetry and Age of the Cassiopeia A Supernova Remnant. ApJ645:283-292, arXiv:astro-ph/0603371

Fiasson A, Kosack K, Skilton J, Gallant Y, et al. (2008) Probing cosmic ray acceleration through molecular clouds in the vicinity of supernova remnant with H.E.S.S. In: American Institute of Physics Conference Series (ed. F A Aharonian, W Hofmann, \& F Rieger), vol. 1085 of American Institute of Physics Conference Series, pp. 361-363

Field GB, Goldsmith DW, Habing HJ (1969) Cosmic-Ray Heating of the Interstellar Gas. ApJ155:L149+

Filippenko AV (1988) Supernova 1987K - Type II in youth, type Ib in old age. AJ96:1941-1948

Filippenko AV (1997) Optical Spectra of Supernovae. ARA\&A35:309-355

Frail DA, Goss WM, Reynoso EM, Giacani EB, et al. (1996) A Survey for OH (1720 MHz) Maser Emission Toward Supernova Remnants. AJ111:1651

Fransson C, Björnsson CI (1998) Radio Emission and Particle Acceleration in SN 1993J. ApJ509:861-878, arXiv:astro-ph/9807030

Fukui Y, Moriguchi Y, Tamura K, Yamamoto H, et al. (2003) Discovery of Interacting Molecular Gas toward the TeV Gamma-Ray Peak of the SNR G 347.3-0.5. PASJ55:L61-L64

Furuzawa A, Ueno D, Hayato A, Ozawa M, et al. (2009) Doppler-Broadened Iron X-Ray Lines From Tycho's Supernova Remnant. ApJ693:L61-L65, 0902.3049

Gabici S, Aharonian FA, Casanova S (2009) Broad-band non-thermal emission from molecular clouds illuminated by cosmic rays from nearby supernova remnants. MNRAS396:1629-1639, 0901.4549 
Genzel R, Eisenhauer F, Gillessen S (2010) The Galactic Center massive black hole and nuclear star cluster. Reviews of Modern Physics 82:3121$3195,1006.0064$

Gerin M, de Luca M, Goicoechea JR, Herbst E, et al. (2010) Interstellar $\mathrm{CH}$ absorption in the diffuse interstellar medium along the sight-lines to G10.6-0.4 (W31C), W49N, and W51. A\&A521:L16+

Ghavamian P, Laming JM, Rakowski CE (2007) A Physical Relationship between Electron-Proton Temperature Equilibration and Mach Number in Fast Collisionless Shocks. ApJ654:L69-L72, arXiv:astro-ph/0611306

Ginzburg VL, Syrovatskii SI (1965) Cosmic Magnetobremsstrahlung (synchrotron Radiation). ARA\&A3:297

Ginzburg VL, Syrovatskij SI (1967) Cosmic rays in the Galaxy (Introductory Report). In: Radio Astronomy and the Galactic System (ed. H van Woerden), vol. 31 of IAU Symposium, pp. 411-+

Giordano F, Naumann-Godo M, Ballet J, Bechtol K, et al. (2011) FermiLAT Detection of the Young SuperNova Remnant Tycho. ArXiv e-prints 1108.0265

Giuliani A, Cardillo M, Tavani M, Fukui Y, et al. (2011) Neutral Pion Emission from Accelerated Protons in the Supernova Remnant W44. ApJ742:L30

Giuliani A, Tavani M, Bulgarelli A, Striani E, et al. (2010) AGILE detection of GeV $\gamma$-ray emission from the SNR W28. A\&A516:L11, 1005.0784

Gloeckler G, Geiss J, Balsiger H, Fisk LA, et al. (1993) Detection of interstellar pick-up hydrogen in the solar system. Science 261:70-73

Gotthelf EV, Koralesky B, Rudnick L, Jones TW, et al. (2001) Chandra Detection of the Forward and Reverse Shocks in Cassiopeia A. ApJ552:L39 L43, arXiv:astro-ph/0104161

Green DA (2009) A Catalogue of Galactic Supernova Remnants (Green, 2009). VizieR Online Data Catalog 7253:0-+

Gull SF (1973) A numerical model of the structure and evolution of young supernovaremnants. MNRAS161:47-69

Hamilton AJS, Sarazin CL, Szymkowiak AE (1986) The X-ray spectrum of SN 1006. ApJ300:698-712

Heiles C (1990) Clustered supernovae versus the gaseous disk and halo. ApJ354:483-491

Helder EA, Kosenko D, Vink J (2010) Cosmic-ray Acceleration Efficiency versus Temperature Equilibration: The Case of SNR 0509-67.5. ApJ719:L140 L144, 1007.3138

Helder EA, Vink J (2008) Characterizing the Nonthermal Emission of Cassiopeia A. ApJ686:1094-1102

Helder EA, Vink J, Bassa CG (2011) Temperature Equilibration Behind the Shock Front: An Optical and X-Ray Study Of RCW 86. ApJ737:85-+, 1106.0303

Helder EA, Vink J, Bassa CG, Bamba A, et al. (2009) Measuring the CosmicRay Acceleration Efficiency of a Supernova Remnant. Science 325:719-, 0906.4553

Helmboldt JF, Kassim NE (2009) The Evolution of Cassiopeia A at Low Radio Frequencies. AJ138:838-844, 0903.5010 
Heng K (2010) Balmer-Dominated Shocks: A Concise Review. PASA 27:23$44,0908.4080$

Heng K, McCray R (2007) Balmer-dominated Shocks Revisited. ApJ654:923937, arXiv: astro-ph/0609331

Heng K, van Adelsberg M, McCray R, Raymond JC (2007) The Transition Zone in Balmer-dominated Shocks. ApJ668:275-284, 0705.2619

Herbst E, Klemperer W (1973) The Formation and Depletion of Molecules in Dense Interstellar Clouds. ApJ185:505-534

Hess VF (1912) Über ber Beobachtungen der durchdringenden Strahlung bei sieben Freiballonfahrten. Physik Zeitschr 13:1084-

HESS Collaboration, Abramowski A, Acero F, Aharonian F, et al. (2011) A new SNR with TeV shell-type morphology: HESS J1731-347. A\&A531:A81, 1105.3206

HESS Collaboration, Abramowski A, Acero F, Aharonian F, et al. (2011a) Discovery of extended VHE \gamma-ray emission from the vicinity of the young massive stellar cluster Westerlund 1. ArXiv e-prints 1111.2043

HESS Collaboration, Abramowski A, Acero F, Aharonian F, et al. (2011b) Revisiting the Westerlund 2 field with the HESS telescope array. A\&A525:A46+, 1009.3012

HESS Collaboration: F A Aharonian (2006) H.E.S.S. observations of the supernova remnant RX J0852.0-4622: shell-type morphology and spectrum of a widely extended VHE gamma-ray source. astro-ph/0612495 astro-ph/0612495

Hester JJ, Raymond JC, Blair WP (1994) The Balmer-dominated northeast limb of the Cygnus loop supernova remnant. ApJ420:721-745

Hewitt JW, Yusef-Zadeh F, Wardle M (2009) Correlation of Supernova Remnant Masers and Gamma-Ray Sources. ApJ706:L270-L274, 0909.2827

Hewitt JW, Yusef-Zadeh F, Wardle M, Roberts DA, et al. (2006) Green Bank Telescope Observations of IC 443: The Nature of OH (1720 MHz) Masers and OH Absorption. ApJ652:1288-1296, arXiv:astro-ph/0602210

Higdon JC, Lingenfelter RE (2005) OB Associations, Supernova-generated Superbubbles, and the Source of Cosmic Rays. ApJ628:738-749

Hillas AM (2005) TOPICAL REVIEW: Can diffusive shock acceleration in supernova remnants account for high-energy galactic cosmic rays? Journal of Physics G Nuclear Physics 31:95--

Hillebrandt W, Niemeyer JC (2000) Type IA Supernova Explosion Models. ARA\&A38:191-230, arXiv:astro-ph/0006305

Hinton JA, Hofmann W (2009) Teraelectronvolt Astronomy. ARA\&A47:523565

Hiraga JS, Uchiyama Y, Takahashi T, Aharonian FA (2005) Spectral properties of nonthermal X-ray emission from the shell-type SNR RX J1713.7 3946 as revealed by XMM-Newton. A\&A431:953-961, arXiv:astro-ph/ 0407401

Hoffman IM, Goss WM, Brogan CL, Claussen MJ (2005) The OH (1720 $\mathrm{MHz})$ Supernova Remnant Masers in W44: MERLIN and VLBA Polarization Observations. ApJ627:803-812, arXiv:astro-ph/0503481

Hofverberg P, Chaves RCG, Fiasson A, Kosack K, et al. (2011a) Discovery of VHE gamma-rays from the vicinity of the shell-type SNR G318.2+0.1 
with H.E.S.S. ArXiv e-prints 1104.5119

Hofverberg P, Chaves RCG, Méhault J, de Naurois M, et al. (2011b) Discovery of VHE gamma-ray emission from the SNR G15.4+0.1 with H.E.S.S. ArXiv e-prints 1112.2901

Högbom JA, Shakeshaft JR (1961) Secular Variation of the Flux Density of the Radio Source Cassiopeia A. Nature189:561-562

Hovey L, Hughes JP (2012) in prep. ApJ

Hoyle F, Fowler WA (1960) Nucleosynthesis in Supernovae. ApJ132:565-+

Hughes JP, Rakowski CE, Decourchelle A (2000) Electron Heating and Cosmic Rays at a Supernova Shock from Chandra X-Ray Observations of $1 \mathrm{E}$ 0102.2-7219. ApJ543:L61-L65, arXiv: astro-ph/0007032

Hwang U, Petre R, Holt SS, Szymkowiak AE (2001) The Thermal X-Rayemitting Shell of Large Magellanic Cloud Supernova Remnant 0540-69.3. ApJ560:742-748, arXiv:astro-ph/0106415

Indriolo N, Blake GA, Goto M, Usuda T, et al. (2010) Investigating the Cosmic-ray Ionization Rate Near the Supernova Remnant IC 443 through $\mathrm{H}^{+}{ }_{3}$ Observations. ApJ724:1357-1365, 1010.3252

Inoue T, Yamazaki R, Inutsuka Si (2010) Two-step Acceleration Model of Cosmic Rays at Middle-aged Supernova Remnants: Universality in Secondary Shocks. ApJ723:L108-L112, 1010.0706

Inui T, Koyama K, Matsumoto H, Tsuru TG (2009) Time Variability of the Neutral Iron Lines from the Sagittarius B2 Region and Its Implication of a Past Outburst of Sagittarius A. PASJ61:241-+

Isenberg PA (1995) Interstellar pickup ions: Not just theory anymore. Reviews of Geophysics 33:623-628

Jokipii JR (1987) Rate of energy gain and maximum energy in diffusive shock acceleration. ApJ313:842-846

Jones TJ, Rudnick L, DeLaney T, Bowden J (2003) The identification of infrared synchrotron radiation from Cassiopeia A. ApJ:in press

Jun BI, Norman ML (1996) On the Origin of Radial Magnetic Fields in Young Supernova Remnants. ApJ472:245-+, arXiv:astro-ph/9606096

Kamae T, Karlsson N, Mizuno T, Abe T, et al. (2006) Parameterization of $\gamma$, $\mathrm{e}^{+/-}$, and Neutrino Spectra Produced by p-p Interaction in Astronomical Environments. ApJ647:692-708, arXiv:astro-ph/0605581

Katagiri H, Tibaldo L, Ballet J, Giordano F, et al. (2011) Fermi Large Area Telescope Observations of the Cygnus Loop Supernova Remnant. ApJ741:44, 1108.1833

Katsuda S, Petre R, Hughes JP, Hwang U, et al. (2010a) X-ray Measured Dynamics of Tycho's Supernova Remnant. ApJ709:1387-1395, 1001.2484

Katsuda S, Petre R, Mori K, Reynolds SP, et al. (2010b) Steady X-ray Synchrotron Emission in the Northeastern Limb of SN 1006. ApJ723:383-392, 1009.0280

Katsuda S, Tsunemi H, Mori K (2008) The Slow X-Ray Expansion of the Northwestern Rim of the Supernova Remnant RX J0852.0-4622. ApJ678:L35-L38, 0803.3266

Katsuta J, Uchiyama Y, Tanaka T, Tajima H, et al. (2012) Fermi-LAT Observation of Supernova Remnant S147. ArXiv e-prints 1204.4703 
Katz B, Waxman E (2008) In which shell-type SNRs should we look for gamma-rays and neutrinos from P-P collisions? Journal of Cosmology and Astro-Particle Physics 1:18-+, 0706.3485

Kelner SR, Aharonian FA, Bugayov VV (2006) Energy spectra of gamma rays, electrons, and neutrinos produced at proton-proton interactions in the very high energy regime. Phys. Rev. D74(3):034018, arXiv:astro-ph/ 0606058

Keohane JW, Reach WT, Rho J, Jarrett TH (2007) A Near-Infrared and XRay Study of W49 B: A Wind Cavity Explosion. ApJ654:938-944, arXiv: astro-ph/0609533

Kirshner RP, Chevalier RA (1977) Spectra of Cassiopeia A. I - Observations. ApJ218:142-147

Kirshner RP, Chevalier RA (1978) The spectrum of Tycho's supernova remnant. A\&A67:267-271

Klepach EG, Ptuskin VS, Zirakashvili VN (2000) Cosmic ray acceleration by multiple spherical shocks. Astroparticle Physics 13:161-172

Knödlseder J (2000) Cygnus OB2 - a young globular cluster in the Milky Way. A\&A360:539-548, arXiv:astro-ph/0007442

Kosenko D, Blinnikov SI, Vink J (2011) Modeling supernova remnants: effects of diffusive cosmic-ray acceleration on the evolution and application to observations. A\&A532:A114+, 1105.5966

Kosenko D, Helder EA, Vink J (2010) The kinematics and chemical stratification of the type Ia supernova remnant 0519-69.0. An XMM-Newton and Chandra study. A\&A519:A11+, 1001.0983

Kosenko D, Vink J, Blinnikov S, Rasmussen A (2008) XMM-Newton Xray spectra of the SNR 0509-67.5: data and models. A\&A490:223-230, 0807.0579

Koyama K, Hyodo Y, Inui T, Nakajima H, et al. (2007) Iron and Nickel Line Diagnostics for the Galactic Center Diffuse Emission. PASJ59:245-255, arXiv: astro-ph/0609215

Koyama K, Kinugasa K, Matsuzaki K, Nishiuchi M, et al. (1997) Discovery of non-thermal X-rays from the northwest shell of the new SNR RX J1713.73946. PASJ49:L7-L11

Koyama K, Maeda Y, Sonobe T, Takeshima T, et al. (1996) ASCA View of Our Galactic Center: Remains of Past Activities in X-Rays? PASJ48:249255

Koyama K, Petre R, Gotthelf EV, Hwang U, et al. (1995) Evidence for Shock Acceleration of High-Energy Electrons in the Supernova Remnant SN:1006. Nature378:255-+

Krause O, Birkmann SM, Usuda T, Hattori T, et al. (2008) The Cassiopeia A Supernova Was of Type IIb. Science 320:1195-, 0805.4557

Krymskii GF (1977) A regular mechanism for the acceleration of charged particles on the front of a shock wave. Akademiia Nauk SSSR Doklady 234:1306-1308

Laffon H, Khélifi B, Brun F, Acero F, et al. (2011) Evidence for VHE emission from SNR G22.7-0.2 region with H.E.S.S. ArXiv e-prints 1110.6890

Lagage PO, Cesarsky CJ (1983) The maximum energy of cosmic rays accelerated by supernova shocks. A\&A125:249-257 
Laming JM (2001) Accelerated electrons in Cassiopeia A: An explanation for the hard X-ray tail. ApJ546:1149-1158

Laming JM, Hwang U (2003) On the Determination of Ejecta Structure and Explosion Asymmetry from the X-Ray Knots of Cassiopeia A. ApJ597:347-361, arXiv: astro-ph/0306119

LaRosa TN, Brogan CL, Shore SN, Lazio TJ, et al. (2005) Evidence of a Weak Galactic Center Magnetic Field from Diffuse Low-Frequency Nonthermal Radio Emission. ApJ626:L23-L27, arXiv: astro-ph/0505244

Lee J, Raymond JC, Park S, Blair WP, et al. (2010) Resolved Shock Structure of the Balmer-dominated Filaments in Tycho's Supernova Remnant: Cosmic-ray Precursor? ApJ715:L146-L149, 1005.3296

Lee JJ, Koo BC, Raymond J, Ghavamian P, et al. (2007) Subaru HDS Observations of a Balmer-dominated Shock in Tycho's Supernova Remnant. ApJ659:L133-L136

Li H, Chen Y (2011) Gamma-rays from molecular clouds illuminated by accumulated diffusive protons. II: interacting supernova remnants. ArXiv e-prints 1108.4541

Li W, Leaman J, Chornock R, Filippenko AV, et al. (2011) Nearby supernova rates from the Lick Observatory Supernova Search - II. The observed luminosity functions and fractions of supernovae in a complete sample. MNRAS412:1441-1472, 1006.4612

Lim AJ, Raga AC (1996) A distribution function calculation of the $\mathrm{H} \alpha$ profiles of high-velocity shocks - II. The broad component neutral precursor. MNRAS280:103-114

Lucek SG, Bell AR (2000) Non-linear amplification of a magnetic field driven by cosmic ray streaming. MNRAS314:65-74

Maddox LA, Williams RM, Dunne BC, Chu YH (2009) Nonthermal Xray Emission in the N11 Superbubble in the Large Magellanic Cloud. ApJ699:911-916, 0904.1821

Malkov MA (1997) Analytic Solution for Nonlinear Shock Acceleration in the Bohm Limit. ApJ485:638-+, arXiv:astro-ph/9707152

Malkov MA (1999) Asymptotic Particle Spectra and Plasma Flows at Strong Shocks. ApJ511:L53-L56, arXiv:astro-ph/9807097

Malkov MA, Diamond PH, Sagdeev RZ (2011) Mechanism for spectral break in cosmic ray proton spectrum of supernova remnant W44. Nature Communications 2, 1004.4714

Malkov MA, Drury L (2001) Nonlinear theory of diffusive acceleration of particles by shock waves. Reports of Progress in Physics64:429-481

Marcaide JM, Martí-Vidal I, Alberdi A, Pérez-Torres MA, et al. (2009) A decade of SN 1993J: discovery of radio wavelength effects in the expansion rate. A\&A505:927-945, 0903.3833

Marcowith A, Lemoine M, Pelletier G (2006) Turbulence and particle acceleration in collisionless supernovae remnant shocks. II. Cosmic-ray transport. A\&A453:193-202, arXiv:astro-ph/0603462

Martí-Vidal I, Pérez-Torres MA, Brunthaler A (2011) Electron cooling and the connection between expansion and flux-density evolution in radio supernovae. A\&A529:A47+, 1101.5380 
McKee CF, Ostriker JP (1977) A theory of the interstellar medium - Three components regulated by supernova explosions in an inhomogeneous substrate. ApJ218:148-169

McKenzie JF, Völk HJ (1982) Non-linear theory of cosmic ray shocks including self-generated Alfven waves. A\&A116:191-200

Méhault J, Clapson AC, Fuessling M, Abramowski A, et al. (2010) Unveiling the origin of gamma-ray emission towards the W41 region with H.E.S.S. data. In: 38th COSPAR Scientific Assembly, vol. 38, p. 2801

Melia F, Fatuzzo M (2011) Diffusive cosmic-ray acceleration at the Galactic Centre. MNRAS410:L23-L27, 1010.2949

Miceli M, Bocchino F, Iakubovskyi D, Orlando S, et al. (2009) Thermal emission, shock modification, and X-ray emitting ejecta in SN 1006 . A\&A501:239-249, 0903.3392

Milne DK (1987) An atlas of supernova remnant magnetic fields. Australian Journal of Physics 40:771-787

Minkowski R (1941) Spectra of Supernovae. PASP53:224-+

Minkowski R (1957) Optical investigations of radio sources (Introductory Lecture). In: Radio astronomy (ed. H C van de Hulst), vol. 4 of IAU Symposium, pp. 107-+

Miyata E, Tsunemi H, Aschenbach B, Mori K (2001) Chandra X-Ray Observatory Study of Vela Shrapnel A. ApJ559:L45-L48

Moebius E, Hovestadt D, Klecker B, Scholer M, et al. (1985) Direct observation of $\mathrm{He}(+)$ pick-up ions of interstellar origin in the solar wind. Nature318:426-429

Morlino G, Caprioli D (2011) Strong evidences of hadron acceleration in Tycho's Supernova Remnant. ArXiv e-prints 1105.6342

Morris M, Serabyn E (1996) The Galactic Center Environment. ARA\&A34:645-702

Muno MP, Baganoff FK, Brandt WN, Park S, et al. (2007) Discovery of Variable Iron Fluorescence from Reflection Nebulae in the Galactic Center. ApJ656:L69-L72, arXiv:astro-ph/0611651

Ng CY, Gaensler BM, Staveley-Smith L, Manchester RN, et al. (2008) Fourier Modeling of the Radio Torus Surrounding SN 1987A. ApJ684:481-497, 0805.4195

Niemiec J, Pohl M, Stroman T, Nishikawa KI (2008) Production of Magnetic Turbulence by Cosmic Rays Drifting Upstream of Supernova Remnant Shocks. ApJ684:1174-1189, 0802.2185

Norman CA, Ikeuchi S (1989) The disk-halo interaction - Superbubbles and the structure of the interstellar medium. ApJ345:372-383

Ohira Y, Murase K, Yamazaki R (2010) Escape-limited model of cosmic-ray acceleration revisited. A\&A513:A17+, 0910.3449

Ohira Y, Murase K, Yamazaki R (2011a) Gamma-rays from molecular clouds illuminated by cosmic rays escaping from interacting supernova remnants. MNRAS410:1577-1582, 1007.4869

Ohira Y, Reville B, Kirk JG, Takahara F (2009a) Two-Dimensional ParticleIn-Cell Simulations of the Nonresonant, Cosmic-Ray-Driven Instability in Supernova Remnant Shocks. ApJ698:445-450, 0812.0901 
Ohira Y, Takahara F (2010) Effects of Neutral Particles on Modified Shocks at Supernova Remnants. ApJ721:L43-L47, 0912.2859

Ohira Y, Terasawa T, Takahara F (2009b) Plasma Instabilities as a Result of Charge Exchange in the Downstream Region of Supernova Remnant Shocks. ApJ703:L59-L62, 0908.3369

Ohira Y, Yamazaki R, Kawanaka N, Ioka K (2011b) Escape of cosmic-ray electrons from supernova remnants. ArXiv e-prints 1106.1810

Oka T, Geballe TR, Goto M, Usuda T, et al. (2005) Hot and Diffuse Clouds near the Galactic Center Probed by Metastable $\mathrm{H}^{+}{ }_{3} 1,$. ApJ632:882-893, arXiv:astro-ph/0507463

Oort JH (1951) Problems in Cosmical Aerodynamics. Central Air Documents Office, Dayton

Ostriker JP, McKee CF (1988) Astrophysical blastwaves. Reviews of Modern Physics 60:1-68

Padovani M, Galli D (2011) Effects of magnetic fields on the cosmic-ray ionization of molecular cloud cores. A\&A530:A109+, 1104.5445

Padovani M, Galli D, Glassgold AE (2009) Cosmic-ray ionization of molecular clouds. A\&A501:619-631, 0904.4149

Parizot E, Marcowith A, Ballet J, Gallant YA (2006) Observational constraints on energetic particle diffusion in young supernovae remnants: amplified magnetic field and maximum energy. A\&A453:387-395, arXiv: astro-ph/0603723

Parizot E, Marcowith A, van der Swaluw E, Bykov AM, et al. (2004) Superbubbles and energetic particles in the Galaxy. I. Collective effects of particle acceleration. A\&A424:747-760, arXiv: astro-ph/0405531

Park S, Hughes JP, Slane PO, Mori K, et al. (2010) A Deep Chandra Observation of the Oxygen-Rich Supernova Remnant 0540-69.3 in the Large Magellanic Cloud. ApJ710:948-957, 0912.5177

Patnaude DJ, Ellison DC, Slane P (2009) The Role of Diffusive Shock Acceleration on Nonequilibrium Ionization in Supernova Remnants. ApJ696:1956-1963, 0902.2481

Patnaude DJ, Fesen RA (2007) Small-Scale X-Ray Variability in the Cassiopeia A Supernova Remnant. AJ133:147-153, arXiv:astro-ph/0609412

Patnaude DJ, Fesen RA (2009) Proper Motions and Brightness Variations of Nonthermal X-ray Filaments in the Cassiopeia A Supernova Remnant. ApJ697:535-543, 0808.0692

Patnaude DJ, Vink J, Laming JM, Fesen RA (2011) A Decline in the Nonthermal X-ray Emission from Cassiopeia A. ApJ729:L28+, 1012.0243

Paumard T, Genzel R, Martins F, Nayakshin S, et al. (2006) The Two Young Star Disks in the Central Parsec of the Galaxy: Properties, Dynamics, and Formation. ApJ643:1011-1035, arXiv:astro-ph/0601268

Petruk O (2005) On the Transition of the Adiabatic Supernova Remnant to the Radiative Stage in a Nonuniform Interstellar Medium. Journal of Physical Studies 9:364-373

Pohl M, Yan H, Lazarian A (2005) Magnetically Limited X-Ray Filaments in Young Supernova Remnants. ApJ626:L101-L104

Ponti G, Terrier R, Goldwurm A, Belanger G, et al. (2010) Discovery of a Superluminal Fe K Echo at the Galactic Center: The Glorious Past of Sgr 
A* Preserved by Molecular Clouds. ApJ714:732-747, 1003.2001

Porquet D, Grosso N, Predehl P, Hasinger G, et al. (2008) X-ray hiccups from Sagittarius $A^{*}$ observed by XMM-Newton. The second brightest flare and three moderate flares caught in half a day. A\&A488:549-557, 0806.4088

Portegies Zwart SF, McMillan SLW, Gieles M (2010) Young Massive Star Clusters. ARA\&A48:431-493, 1002.1961

Ptuskin V, Zirakashvili V, Seo ES (2010) Spectrum of Galactic Cosmic Rays Accelerated in Supernova Remnants. ApJ718:31-36, 1006.0034

Ptuskin VS, Zirakashvili VN (2005) On the spectrum of high-energy cosmic rays produced by supernova remnants in the presence of strong cosmicray streaming instability and wave dissipation. A\&A429:755-765, arXiv: astro-ph/0408025

Quataert E, Loeb A (2005) Nonthermal THz to TeV Emission from Stellar Wind Shocks in the Galactic Center. ApJ635:L45-L48, arXiv:astro-ph/ 0509265

Rakowski CE, Laming JM, Ghavamian P (2008) The Heating of Thermal Electrons in Fast Collisionless Shocks: The Integral Role of Cosmic Rays. ApJ684:348-357, 0805.3084

Rakowski CE, Laming JM, Hwang U, Eriksen KA, et al. (2011) The Effect of a Cosmic Ray Precursor in SN 1006? ApJ735:L21+, 1104.3736

Raymond JC (1976) Theoretical Models of Shock Waves in the Interstellar Medium. Ph.D. thesis, THE UNIVERSITY OF WISCONSIN - MADISON.

Raymond JC (1979) Shock waves in the interstellar medium. ApJS39:1-27

Raymond JC (1991) Supernova-remnant shock waves close up. PASP103:781-786

Raymond JC, Isenberg PA, Laming JM (2008) Non-Maxwellian Proton Velocity Distributions in Nonradiative Shocks. ApJ682:408-415, 0804.3808

Raymond JC, Vink J, Helder EA, de Laat A (2011) Effects of Neutral Hydrogen on Cosmic-ray Precursors in Supernova Remnant Shock Waves. ApJ731:L14+, 1103.3211

Raymond JC, Winkler PF, Blair WP, Lee J, et al. (2010) Non-Maxwellian $\mathrm{H} \alpha$ Profiles in Tycho's Supernova Remnant. ApJ712:901-907

Reed JE, Hester JJ, Fabian AC, Winkler PF (1995) The three-dimensional structure of the Cassiopeia A supernova remnant. I. The spherical shell. ApJ440:706-721

Renaud M, et al. (2006) The Signature of ${ }^{44} \mathrm{Ti}$ in Cassiopeia A Revealed by IBIS/ISGRI on INTEGRAL. ApJ647:L41-L44

Reville B, Bell AR (2011) A filamentation instability for streaming cosmicrays. ArXiv e-prints 1109.5690

Reville B, Kirk JG, Duffy P (2009) Steady-State Solutions in Nonlinear Diffusive Shock Acceleration. ApJ694:951-958, 0812.3993

Revnivtsev MG, Churazov EM, Sazonov SY, Sunyaev RA, et al. (2004) Hard $\mathrm{X}$-ray view of the past activity of $\mathrm{Sgr} \mathrm{A}^{*}$ in a natural Compton mirror. A\&A425:L49-L52, arXiv:astro-ph/0408190

Reynolds SP (1998) Models of synchrotron X-rays from shell supernova remnants. ApJ493:375-+

Reynolds SP (2008) Supernova Remnants at High Energy. ARA\&A46:89-126 
Reynolds SP, Borkowski KJ, Green DA, Hwang U, et al. (2008) The Youngest Galactic Supernova Remnant: G1.9+0.3. ApJ680:L41-L44, 0803.1487

Reynolds SP, Borkowski KJ, Hwang U, Hughes JP, et al. (2007) A Deep Chandra Observation of Kepler's Supernova Remnant: A Type Ia Event with Circumstellar Interaction. ApJ668:L135-L138, arXiv:0708.3858

Reynolds SP, Chevalier RA (1981) Nonthermal Radiation from Supernova Remnants in the Adiabatic Stage of Evolution. ApJ245:912-+

Reynolds SP, Ellison DC (1992) Electron acceleration in Tycho's and Kepler's supernova remnants - Spectral evidence of Fermi shock acceleration. ApJ399:L75-L78

Reynolds SP, Gaensler BM, Bocchino F (2011) Magnetic Fields in Supernova Remnants and Pulsar-Wind Nebulae. Space Sci. Rev.:269-+1104.4047

Reynolds SP, Keohane JW (1999) Maximum energies of shock-accelerated electrons in young shell supernova remnants. ApJ525:368-374

Rho J, Dyer KK, Borkowski KJ, Reynolds SP (2002) X-Ray Synchrotronemitting Fe-rich Ejecta in Supernova Remnant RCW 86. ApJ581:11161131, arXiv:astro-ph/0208013

Rosado M, Le Coarer E, Georgelin YP (1994) Kinematics of supernova remnants in the Small Magellanic Cloud I. The SNRs inside the nebular complex N 19, the SNR 0046-73.5 and the SNR 0050-72.8. A\&A286:231-242

Rothenflug R, et al. (2004) Geometry of the non-thermal emission in SN 1006. A\&A425:121-131

Salvesen G, Raymond JC, Edgar RJ (2009) Shock Speed, Cosmic Ray Pressure, and Gas Temperature in the Cygnus Loop. ApJ702:327-339, 0812.2515

Schlegel EM (1990) A new subclass of Type II supernovae? MNRAS244:269 271

Schure KM, Achterberg A, Keppens R, Vink J (2010) Time-dependent particle acceleration in supernova remnants in different environments. MNRAS406:2633-2649, 1004.2766

Schure KM, Bell AR (2011) A long-wavelength instability involving the stress tensor. MNRAS:1451-+1107.5817

Schure KM, Bell AR, O'C Drury L, Bykov AM (2012) Diffusive Shock Acceleration and Magnetic Field Amplification. Space Sci. Rev.:14 1203.1637

Schure KM, Kosenko D, Kaastra JS, Keppens R, et al. (2009) A new radiative cooling curve based on an up-to-date plasma emission code. A\&A508:751$757,0909.5204$

Schure KM, Vink J, García-Segura G, Achterberg A (2008) Jets as Diagnostics of the Circumstellar Medium and the Explosion Energetics of Supernovae: The Case of Cassiopeia A. ApJ686:399-407, 0806.4617

Shklovskii IS (1953) Reports of the Academy, USSR 90:983

Shklovsky JS (1968) Supernovae. Interscience Monographs and Texts in Physics and Astronomy, London: Wiley, 1968

Slane P, Gaensler BM, Dame TM, Hughes JP, et al. (1999) Nonthermal X-Ray emission from the shell-type supernova remnant G347.3-0.5. ApJ525:357-367

Slane P, et al. (2001) RX J0852.0-4622: Another nonthermal shell-type supernova remnant (G266.2-1.2). ApJ548:814-819 
Smith RC, Kirshner RP, Blair WP, Winkler PF (1991) Six Balmer-dominated supernova remnants. ApJ375:652-662

Sollerman J, Ghavamian P, Lundqvist P, Smith RC (2003) High resolution spectroscopy of Balmer-dominated shocks in the RCW 86, Kepler and SN 1006 supernova remnants. A\&A407:249-257, arXiv:astro-ph/0306196

Spitzer L (1978) Physical processes in the interstellar medium

Stephenson FR, Green DA (2002) Historical supernovae and their remnants, vol. 5

Su M, Slatyer TR, Finkbeiner DP (2010) Giant Gamma-ray Bubbles from Fermi-LAT: Active Galactic Nucleus Activity or Bipolar Galactic Wind? ApJ724:1044-1082, 1005.5480

Sunyaev RA, Markevitch M, Pavlinsky M (1993) The center of the Galaxy in the recent past - A view from GRANAT. ApJ407:606-610

Tammann GA, Loeffler W, Schroeder A (1994) The Galactic supernova rate. ApJS92:487-493

Tanaka T, Allafort A, Ballet J, Funk S, et al. (2011) Gamma-Ray Observations of the Supernova Remnant RX J0852.0-4622 with the Fermi Large Area Telescope. ApJ740:L51+, 1109.4658

Tatischeff V (2009) Radio emission and nonlinear diffusive shock acceleration of cosmic rays in the supernova SN 1993J. A\&A499:191-213, 0903.2944

Tavani M, Barbiellini G, Argan A, Bulgarelli A, et al. (2008) The AGILE space mission. Nuclear Instruments and Methods in Physics Research A 588:52-62

Tavani M, Giuliani A, Chen AW, Argan A, et al. (2010) Direct Evidence for Hadronic Cosmic-Ray Acceleration in the Supernova Remnant IC 443. ApJ710:L151-L155, 1001.5150

Terrier R, Ponti G, Bélanger G, Decourchelle A, et al. (2010) Fading Hard X-ray Emission from the Galactic Center Molecular Cloud Sgr B2. ApJ719:143-150

The LS, et al. (1996) CGRO/OSSE observations of the Cassiopeia A SNR. A\&AS120:C357+

Tian WW, Li Z, Leahy DA, Wang QD (2007) VLA and XMM-Newton Observations of the SNR W41/TeV Gamma-Ray Source HESS J1834-087. ApJ657:L25-L28, arXiv:astro-ph/0612296

Torres DF, Domingo-Santamaría E, Romero GE (2004) High-Energy Gamma Rays from Stellar Associations. ApJ601:L75-L78, arXiv:astro-ph/ 0312128

Torres DF, Li H, Chen Y, Cillis A, et al. (2011) Cosmic rays in the surroundings of SNR G35.6-0.4. MNRAS417:3072-3079, 1107.3470

Truelove JK, McKee CF (1999) Evolution of Nonradiative Supernova Remnants. ApJS120:299-326

Uchiyama Y, Aharonian FA (2008) Fast Variability of Nonthermal X-Ray Emission in Cassiopeia A: Probing Electron Acceleration in ReverseShocked Ejecta. ApJ677:L105-L108, arXiv:0803.3410

Uchiyama Y, Aharonian FA, Tanaka T, Takahashi T, et al. (2007) Extremely fast acceleration of cosmic rays in a supernova remnant. Nature449:576 578 
Uchiyama Y, Blandford RD, Funk S, Tajima H, et al. (2010) Gamma-ray Emission from Crushed Clouds in Supernova Remnants. ApJ723:L122L126, 1008.1840

Valinia A, Tatischeff V, Arnaud K, Ebisawa K, et al. (2000) On the Origin of the Iron K Line in the Spectrum of The Galactic X-Ray Background. ApJ543:733-739, arXiv:astro-ph/0006202

van Adelsberg M, Heng K, McCray R, Raymond JC (2008) Spatial Structure and Collisionless Electron Heating in Balmer-dominated Shocks. ApJ689:1089-1104, 0803.2521

van den Bergh S (1971) Optical Studies of Cassiopeia a. III. Spectra of the Supernova Remnant. ApJ165:457-+

van der Laan H (1962) Expanding supernova remnants and galactic radio sources. MNRAS124:125-+

van Marle AJ, Langer N, García-Segura G (2004) Wind-Blown Bubbles around Massive Stars. The effects of stellar wind and photo-ionization on the circumstellar Medium. In: Revista Mexicana de Astronomia y Astrofisica Conference Series (ed. G Garcia-Segura, G Tenorio-Tagle, J Franco, \& H W Yorke ), vol. 22 of Revista Mexicana de Astronomia y Astrofisica Conference Series, pp. 136-139

van Veelen B, Langer N, Vink J, García-Segura G, et al. (2009) The hydrodynamics of the supernova remnant Cassiopeia A. The influence of the progenitor evolution on the velocity structure and clumping. A\&A503:495503, 0907.1197

van Weeren RJ, Röttgering HJA, Brüggen M, Hoeft M (2010) Particle Acceleration on Megaparsec Scales in a Merging Galaxy Cluster. Science $330: 347-, 1010.4306$

Vink J (2004) A Review of X-ray Observations of Supernova Remnants. Nuclear Physics B Proceedings Supplements 132:21-30, arXiv:astro-ph/ 0311406

Vink J (2005) Non-thermal X-ray Emission from Supernova Remnants. In: AIP Conf. Proc. 745: High Energy Gamma-Ray Astronomy (eds. Aharonian FA, Völk HJ, Horns D), pp. 160-171

Vink J (2006) X-ray High Resolution and Imaging Spectroscopy of Supernova Remnants. In: The X-ray Universe 2005, ESA SP-604 Vol. 1, A. Wilson ed. (ESA, ESTEC, The Netherlands), p. 319

Vink J (2008a) Multiwavelength Signatures of Cosmic Ray Acceleration by Young Supernova Remnants. In: HIGH ENERGY GAMMA-RAY ASTRONOMY: Proceedings of the 4th International Meeting on High Energy Gamma-Ray Astronomy., vol. 1085 of AIP, pp. 169-180

Vink J (2008b) Non-thermal bremsstrahlung from supernova remnants and the effect of Coulomb losses. A\&A486:837-841, arXiv:0806.4393

Vink J (2008c) The Kinematics of Kepler's Supernova Remnant as Revealed by Chandra. ApJ689:231-241, 0803.4011

Vink J, Bleeker J, van der Heyden K, Bykov A, et al. (2006) The XRay Synchrotron Emission of RCW 86 and the Implications for Its Age. ApJ648:L33-L37

Vink J, Bloemen H, Kaastra JS, Bleeker JAM (1998) The expansion of Cassiopeia A as seen in X-rays. A\&A339:201-207 
Vink J, Kaastra JS, Bleeker JAM (1997) X-ray spectroscopy of the supernova remnant RCW 86. A new challenge for modeling the emission from supernova remnants. A\&A328:628-633

Vink J, Laming JM (2003) On the Magnetic Fields and Particle Acceleration in Cassiopeia A. ApJ584:758-769, arXiv:astro-ph/0210669

Vink J, Yamazaki R, Helder EA, Schure KM (2010) The Relation Between Post-shock Temperature, Cosmic-ray Pressure, and Cosmic-ray Escape for Non-relativistic Shocks. ApJ722:1727-1734, 1008.4367

Vink J, et al. (2001) Detection of the 67.9 and $78.4 \mathrm{keV}$ lines associated with the radioactive decay of ${ }^{44} \mathrm{Ti}$ in Cassiopeia A. ApJ560:L79-L82

Vladimirov A, Ellison DC, Bykov A (2006) Nonlinear Diffusive Shock Acceleration with Magnetic Field Amplification. ApJ652:1246-1258, arXiv: astro-ph/0606433

Vladimirov AE, Bykov AM, Ellison DC (2008) Turbulence Dissipation and Particle Injection in Nonlinear Diffusive Shock Acceleration with Magnetic Field Amplification. ApJ688:1084-1101, 0807.1321

Vladimirov AE, Bykov AM, Ellison DC (2009) Spectra of Magnetic Fluctuations and Relativistic Particles Produced by a Nonresonant Wave Instability in Supernova Remnant Shocks. ApJ703:L29-L32, 0908.2602

Völk HJ, Berezhko EG, Ksenofontov LT (2005) Magnetic field amplification in Tycho and other shell-type supernova remnants. A\&A433:229-240

Wagner AY, Lee JJ, Raymond JC, Hartquist TW, et al. (2009) A CosmicRay Precursor Model for a Balmer-Dominated Shock in Tycho's Supernova Remnant. ApJ690:1412-1423, 0809.2504

Wang CY (2011) Rayleigh-Taylor instabilities in Type Ia supernova remnants undergoing cosmic ray particle acceleration - low adiabatic index solutions. MNRAS415:83-92, 1011.3129

Wang ZR, Qu QY, Chen Y (1997) Is RX J1713.7-3946 the remnant of the AD393 guest star? A\&A318:L59-L61

Wardle M, Yusef-Zadeh F (2002) Supernova Remnant OH Masers: Signposts of Cosmic Collision. Science 296:2350-2354

Warren JS, Hughes JP, Badenes C, Ghavamian P, et al. (2005) CosmicRay Acceleration at the Forward Shock in Tycho's Supernova Remnant: Evidence from Chandra X-Ray Observations. ApJ634:376-389, arXiv: astro-ph/0507478

Webber WR (1998) A New Estimate of the Local Interstellar Energy Density and Ionization Rate of Galactic Cosmic Cosmic Rays. ApJ506:329-334

Weekes TC, Cawley MF, Fegan DJ, Gibbs KG, et al. (1989) Observation of $\mathrm{TeV}$ gamma rays from the Crab nebula using the atmospheric Cerenkov imaging technique. ApJ342:379-395

Weiler KW, Panagia N, Montes MJ, Sramek RA (2002) Radio Emission from Supernovae and Gamma-Ray Bursters. ARA\&A40:387-438

Westerlund BE (1969) OB stars near the supernova remnant RCW 86. AJ74:879-881

Willingale R, West RG, Pye JP, Stewart GC (1996) ROSAT PSPC observations of the remnant of SN 1006. MNRAS278:749-762

Winkler PF, Gupta G, Long KS (2003) The SN 1006 Remnant: Optical Proper Motions, Deep Imaging, Distance, and Brightness at Maximum. 
ApJ585:324-335

Wommer E, Melia F, Fatuzzo M (2008) Diffuse TeV emission at the Galactic Centre. MNRAS387:987-997, 0804.3111

Wright NJ, Drake JJ (2009) The Massive Star-Forming Region Cygnus OB2. I. Chandra Catalog of Association Members. ApJS184:84-99, 0908.0549

Wu JHK, Wu EMH, Hui CY, Tam PHT, et al. (2011) Discovery of GammaRay Emission from the Supernova Remnant Kes 17 with Fermi Large Area Telescope. ApJ740:L12, 1108.4084

Yanasak NE, Wiedenbeck ME, Mewaldt RA, Davis AJ, et al. (2001) Measurement of the Secondary Radionuclides ${ }^{10} \mathrm{Be},{ }^{26} \mathrm{Al},{ }^{36} \mathrm{Cl},{ }^{54} \mathrm{Mn}$, and ${ }^{14} \mathrm{C}$ and Implications for the Galactic Cosmic-Ray Age. ApJ563:768-792

Yungelson LR, van den Heuvel EPJ, Vink JS, Portegies Zwart SF, et al. (2008) On the evolution and fate of super-massive stars. A\&A477:223-237, 0710.1181

Yusef-Zadeh F, Muno M, Wardle M, Lis DC (2007) The Origin of Diffuse X-Ray and $\gamma$-Ray Emission from the Galactic Center Region: Cosmic-Ray Particles. ApJ656:847-869, arXiv:astro-ph/0608710

Zirakashvili VN, Aharonian F (2007) Analytical solutions for energy spectra of electrons accelerated by nonrelativistic shock-waves in shell type supernova remnants. A\&A465:695-702, arXiv: astro-ph/0612717

Zirakashvili VN, Aharonian FA (2010) Nonthermal Radiation of Young Supernova Remnants: The Case of RX J1713.7-3946. ApJ708:965-980, 0909.2285

Zirakashvili VN, Ptuskin VS (2008) Diffusive Shock Acceleration with Magnetic Amplification by Nonresonant Streaming Instability in Supernova Remnants. ApJ678:939-949, 0801.4488

Zubovas K, King AR, Nayakshin S (2011) The Milky Way's Fermi bubbles: echoes of the last quasar outburst? MNRAS415:L21-L25, 1104.5443 